\title{
INVESTIGATIONS OF THE DYNAMICS AND ELECTROMAGNETIC INTERACTIONS OF FEW-BODY SYSTEMS
}

Progress Report

30 June 1994 to 30 September 1995

\author{
Principal Investigator \\ Donald R. Lehman \\ Co-Principal Investigators \\ Cornelius Bennhold \\ Helmut Haberzettl \\ Leonard C. Maximon \\ William C. Parke \\ Senior Investigators \\ Hiroshi Ito \\ Graduate Students \\ Reyna Kushner Pratt \\ Mohamed Najmeddine \\ Ali Rakei
} RECEIVED JAII 07 9997 $08 T$

Center for.Nuclear Studies

The George Washington University

Washington, DC 20052

October 1995

PREPARED FOR THE U.S. DEPARTMENT OF ENERGY

UNDER GRANT NUMBER

DE-FG02-95-ER409074027d

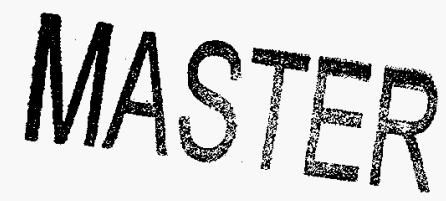




\section{DISCLAMMIER}

Portions of this document may be illegible in electronic image products. Images are produced from the best available original document. 


\section{DISCLAIMER}

This report was prepared as an account of work sponsored by an agency of the United States Government. Neither the United States Government nor any agency thereof, nor any of their employees, makes any warranty, express or implied, or assumes any legal liability or responsibility for the accuracy, completeness, or usefulness of any information, apparatus, product, or process disclosed, or represents that its use would not infringe privately owned rights. Reference herein to any specific commercial product, process, or service by trade name, trademark, manufacturer, or otherwise does not necessarily constitute or imply its endorsement, recommendation, or favoring by the United States Government or any agency thereof. The views and opinions of authors expressed herein do not necessarily state or reflect those of the United States Government or any agency thereof. 
PROGRESS -- DE-FG02-95-ER4090i

\section{INTRODUCTION}

This progress report summarizes the work of The George Washington University (GW) nuclear theory group during the period 1 July 1994 - 30 September 1995 under DOE Grant No. DE-FG02-95-ER40907. During this period, the faculty working under the grant were D.R. Lehman (P.I.), C. Bennhold, H. Haberzettl, L.C. Maximon, and W.C. Parke (co-PI's), and H. Ito (Senior Investigator). Three graduate students, Reyna Kushner Pratt, Mohamed Najmeddine, and Ali Rakei, all supported by the grant, joined the effort as of 1 June 1992 through a supplemental addition of funds from DOE and tuition cost-sharing by GW. Ms. Kushner, who passed her general examinations during the 1993-94 academic year, is working with Professor Bennhold. Mr. Najmeddine and Mr. Rakei both passed their general examinations more than two years ago and are working with Professors Lehman and Haberzettl, respectively. During the summer period, we usually support one or two graduate students for a three-month period to provide them with the opportunity to learn about theoretical nuclear physics, to do a small research project, and to become acquainted with our group. This summer (1995), we supported one student (Louis Alfieri) who worked with Professor Bennhold on $(\gamma, \mathrm{K})$ processes. Outside collaborators with the group during the period of the progress report were B.F. Gibson of Los Alamos National Laboratory, F. Gross of William and Mary/CEBAF, A. Eiro and A. Fonseca of the U. of Lisbon, J. Haidenbauer of Hannover University, S.S. Kamalov of Dubna, Y. Koike of Hosei University (Tokyo), A. Ramos from Barcelona, L. Tiator from the University of Mainz, J. Tjon of the University of Utrecht, and $H$. Weller of Duke University.

The format of the Progress Report is as follows:

1. Papers published or in press, submitted for publication, and in preparation;

2. Invited talks at conferences and meetings;

3. Invited talks at universities and laboratories;

4. Contributed papers or abstracts at conferences;

5. Visitors to the group;

6. Research progress by topic. 
PROGRESS -- DE-FG02-95-ER40907

\section{PAPERS PUBLISHED}

H.R. Weller, R.M. Chasteler, B.S. Marks, R.G. Seyler, and D.R. Lehman, "Angular Distribution Coefficients for $(\gamma, \mathrm{X})$ Reactions with Circularly Polarized Photons and Polarized Targets and a Correction to Previous Tables", Atomic Data and Nuclear Data Tables $\underline{58}, 219$ (1994).

B.F. Gibson, I.R. Afnan, J.A. Carlson, and 'D.R. Lehman, "Importance of BaryonBaryon Coupling in Hypernuclei”, Prog. Theor. Phys. Suppl. 117, 339 (1994).

A. Ramos, C. Bennhold, "Weak Decay of Polarized A-Hypernuclei", Nucl. Phys. A577, 287 (1994).

Xiaodong Li, L.E. Wright, C. Bennhold, "Exclusive Radiative Pion Capture from Nuclei in the $\Delta$-Region", Phys. Rev. C므, R1283 (1994).

L. Tiator, C. Bennhold, S.S. Kamalov, "The $\eta N N$ Coupling Constant in Eta Photoproduction", Nucl. Phys. A580, 455 (1994).

D.R. Lehman, H.R. Weller, and R.M. Whitton, "Production of E1 Radiation in the ${ }^{2} \mathrm{H}(\mathrm{d}, \gamma){ }^{4} \mathrm{He}$ Reaction at Very Low Energies, Few-Body Systems 18, 147 (1995).

H. Haberzettl, "Covariant Off-Shell Model for Dynamical Quark Confinement", Nucl. Phys. A582, 603 (1995).

H. Ito, "Flavor $\mathrm{SU}(3)$ symmetry in the anomalous magnetic moments of constituent quarks", Phys. Lett. B353, 13 (1995).

W.W. Buck, R.A. Williams, and H. Ito, "Elastic charge form factors for $K$ mesons", Phys. Lett. B $\underline{351}, 24$ (1995).

T. Mart, C. Bennhold, C. Hyde-Wright, "Suppression of Coupling Constants in Charged $\Sigma$ Photoproduction", Phys. Rev. C 51 , R1077 (1995).

S.K. Matthews, W.J. Briscoe, C. Bennhold, B.L. Berman, R.W. Caress, K.S. Dhuga, S.N: Dragic, N.J. Nicholas, M.R. Taragin, L. Tiator; S.S. Kamalov, S.J. Greene, D.B. Barlow, B.M.K. Nefkens, C. Pillai, J.W. Price, L.D. Isenhower, M.E. Sadler, I. Slaus, I. Supek, "Elastic Scattering of Pions from ${ }^{3} \mathrm{H}$ and ${ }^{3} \mathrm{He}$ into the Backward Hemisphere", Phys. Rev. Cㅌ1, 2534 (1995).

S.S. Kamalov, L. Tiator, C. Bennhold, "Two-Body Mechanisms in Pion Photoproduction on the Trinucleon", Phys. Rev. Lett. $\underline{75}, 1288$ (1995)

H. Ito, "Strangeness form factors of the nucleon and the anomalous magnetic moments of constituent quarks"; Phys. Rev. C토, R1750 (1995).

A. Parreno, A. Ramos, C. Bennhold, "The Role of the Rho Meson in the Nonmesonic Hypernuclear Decay", Phys. Rev. C (in press) 
PROGRESS -- DE-FG02-95-ER40907

\section{PAPERS SUBMITTED}

C. Bennhold, H. Ito, T. Mart, "The $\mathrm{K}^{0}$ Form Factor in Kaon Electroproduction". submitted to Phys. Rev. Lett.

S.S. Kamalov, L. Tiator, C. Bennhold, "Elastic Pion Scattering on the Deuteron". submitted to Phys. Rev. C.

S.S. Kamalov, L. Tiator, C. Bennhold, "Coherent Pion and Eta Photoproduction on the Deuteron", submitted to Phys. Rev. C.

L.C. Maximon, "Bethe's Bremsstrahlung Spectrum Revisited", submitted to Phys. Rev. $\mathrm{C}$.

Mohamed Najmeddine, "Three-Body Bound State Calculations using Separable Expansion of the Two-Body Potential", submitted to Phys. Rev. C.

\section{PAPERS IN PREPARATION}

D.R. Lehman and B.F. Gibson, "Formalism Underlying the $A=4 \Lambda$ Hypernuclei $1^{+}$ Equations", to be submitted to Phys. Rev. C.

Y. Koike, D.R. Lehman, L.C. Maximon, and W.C. Parke, "A Possible Treatment of Three-Body Bound States with Long Range Interactions", to be submitted to Phys. Rev. C.

Y. Koike, D.R. Lehman, W.C. Parke, and L.C. Maximon, "Highly Accurate Threenucleon Bound-state Calculation with a Unified Separable Expansion of the Two-body T-matrix", to be submitted to Phys. Rev. C.

A.C. Fonseca, J. Haidenbauer, D.R. Lehman, W.C. Parke, and L.C. Maximon, "Eighteen-Channel Three-Nucleon Bound-State Wave Functions from a Separable Expansion Method", to be submitted to the Phys. Rev.

A.C. Fonseca and D.R. Lehman, "Radiative Capture of Polarized Deuterons on Protons", to be submitted to Few-Body Systems.

L.C. Maximon, "A Simple Algorithm for the Calculation of the Spence Function for Complex Arguments in Multiple Precision", to be submitted to Jour. Math. Phys.

Frank X. Lee, L.E. Wright, C. Bennhold, L. Tiator, "Quasifree Eta Photoproduction from Nuclei", to be submitted to Phys. Rev. C.

Frank X. Lee, L.E. Wright, C. Bennhold, "Quasifree Exclusive Pion Electroproduction in the $\Delta$-Region", to be submitted to Phys. Rev. C.

S. Karataglidis, K. Amos, C. Bennhold, L. Tiator, "Large Basis Shell Model Analysis in the Reaction ${ }^{14} \mathrm{~N}\left(\gamma, \pi^{+}\right){ }^{14} \mathrm{C}$ ", to be submitted to Phys. Rev. C. 
PROGRESS -- DE-FG02-95-ER4090†

H. Haberzettl, C. Hanhart, and S. Krewald, "Calculating Meson Masses within a Consistent Model of Dynamically Confined Quarks", to be submitted to Nucl. Phys. A.

\section{INVITED TALKS AT CONFERENCES AND MEETINGS}

H. Haberzettl, "Relativistic Cluster Dynamics of Nucleons and Mesons", in Proceedings of the International Conference on Nucleons and Mesons at Intermediate Energies (Dubna, Russia, May 1994), edited by M.Kh. Khankhasayev and Zh.B. Kurmanov, World Scientific, Singapore (1995), p. 222.

H. Haberzettl and W.C. Parke, "Relativistic Origin of Three-Body Forces", in Few Body Problems in Physics, edited by Franz Gross (American Institute of Physics Conference Proceedings [Williamsburg, VA, May 1994], No. 334, New York, 1995), p. 871.

A.C. Fonseca and D.R. Lehman, "Capture of Polarized Deuterons on Protons", in Few Body Problems in Physics, edited by Franz Gross (American Institute of Physics Conference Proceedings [Williamsburg, VA, May 1994], No. 334, New York, 1995), p. 478.

Y. Koike, D.R. Lehman, L.C. Maximon, and W.C. Parke, "Accurate Triton Calculation with a Local Realistic N-N Interaction with a Separable Expansion Method", in Few Body Problems in Physics, edited by Franz Gross (American Institute of Physics Conference Proceedings [Williamsburg, VA, May 1994], No. 334, New York, 1995), p. 836.

H. Ito, "Octet of Goldstone Bosons and the Electroweak Form Factors of Nucleon". in Few Body Problems in Physics, edited by Franz Gross (American Institute of Physics Conference Proceedings [Williamsburg, VA, May 1994], No. 334, New York, 1995), p. 587.

C. Bennhold, "Photo- and Electroproduction of Strange Particles", Annual DNP-APS Fall Meeting, Williamsburg, Virginia, Oct. 26-29, 1994

C. Hanhart, S. Krewald, H. Haberzettl, I.R. Afnan, and B.F. Gibson, "Faddeev Approach to the Octet and Decuplet Baryons", in Verhandlungen der Deutschen Physikalischen Gesellschaft (Spring Meeting, Cologne, Germany, March 1995), Verh. DPG (VI) $\underline{31}, 646$ (1995).

C. Bennhold, "Electromagnetic Production of Etas on Nucleons and Nuclei", Sixth International Symposium on Meson-Nucleon Physics and the Structure of the Nucleon, Blaubeuren, Germany, 10-14 July 1995.

C. Bennhold, "The Hypernuclear Weak Decay", Sixth International Conference on Mesons and Light Nuclei, Straz pod Ralskem, Czech Republic, 3-7 July 1995.

C. Bennhold, "The Weak Decay of Hypernuclei", Institute for Nuclear Theory Program "Physics beyond the Standard Model at Low and Intermediate Energies", Seattle, WA, 26 June - 1 September 1995. 


\section{Progress Report}

1 July 1991 to 30 June 1994

\section{Principal Investigator}

Donald R. Lehman

Co-Principal Investigators

Helmut Haberzettl

Leonard C. Maximon

William C. Parke

Senior Investigators

Cornelius Bennhold Hiroshi Ito

\section{Graduate Students}

Reyna Kushner Pratt Mohamed Najmeddine Ali Rakei

Center for Nuclear Studies The George Washington University

Washington, DC 20052

July 1994

PREPARED FOR THE U.S. DEPARTMENT OF ENERGY

UNDER GRANT NUMBER

DE-FG05-86-ER40270 
PROGRESS

\section{INIRODUCTION}

This progress report summarizes the work of The George Washington University (GW) nuclear theory group during the period 1 July 1991 - 30 June 1994 under DOE Grant No. DE-FG05-86-ER40270. During this period, the faculty working under the grant were D.R. Lehman (P.I.), H. Haberzettl, L.C. Maximon, and W.C. Parke (co-PI's), and C. Bennhold and H. Ito (Senior Investigators). Professor Bennhold was added to the group during the Fall semester of 1992 after joining the Physics Department faculty in September 1992 as a new assistant professor of physics in theoretical nuclear physics. Hiroshi Ito, who had been a postdoctoral research scientist on the grant beginning 1 Julv 1991, this year became a senior investigator on the grant for the two-year period ending 31 August 1995 while he holds the position of visiting assistant professor of physics. Three graduate students, Reyna Kushner Pratt, Mohamed Najmeddine, and Ali Rakei, all supported by the grant, joined the effort as of 1 June 1992 through a supplemental addition of funds from DOE and tuition cost-sharing by GW. Ms. Kushner passed her general examinations this past academic year, but has been working with Professors Maximon and Bennhold to prepare herself for theoretical work in nuclear physics. Most likely, she will become the student of Professor Bennhold. Mr. Najmeddine and Mr. Rakei both passed their general examinations over a year ago and have chosen to work with Professors Lehman and Haberzettl, respectively. During the summer period, we usually support one or two graduate students for a three-month period to provide them with the opportunity to learn about theoretical nuclear physics, to do a small research project, and to become acquainted with our group. This summer (1994), we are supporting one student (Jijin Yan) who is working with Professors Bennhold and Haberzettl. Outside collaborators with the group for different segments of time during the period indicated were B.F. Gibson of Los Alamos National Laboratory, F. Gross of William and Mary/CEBAF, A. Eiro and A. Fonseca of the U: of Lisbon, J. Haidenbauer of Hannover University, S.S. Kamalov of Dubna, Y. Koike of Hosei University (Tokyo), A. Ramos from Barcelona, and L. Tiator from the University of Mainz, J. Tjon of the University of Utrecht, W. Sandhas of the U. of Bonn, and H. Weller of Duke University.

The format of the Progress Report is as follows:

1. Papers published or in press, submitted for publication, and in preparation;

2. Invited talks at conferences and meetings;

3. Invited talks at universities and laboratories;

4. Contributed papers or abstracts at conferences;

5. Visitors to the group;

6 . Research progress by topic. 
PROGRESS

\section{PAPERS PUBLISHED}

A.C. Fonseca and D.R. Lehman, "Exact Three-Body Calculation of Polarization Observables in ${ }^{1} \mathrm{H}(\overrightarrow{\mathrm{d}}, \gamma){ }^{3} \mathrm{He}$, Phys. Lett. B267,159(1991).

W.C. Parke, Y. Koike, D.R. Lehman, and L.C. Maximon, "Quality of the ThreeNucleon Bound-State Wave Function from a Two-Nucleon Separable Expansion Method", Few-Body Systems 11,89(1991).

H.R. Weller, J. Langenbrunner, R.M. Chasteler, E.L. Tomusiak, J. Asai, R.G. Seyler, and D.R. Lehman, "Angular Distribution Coefficients for $(\gamma, x)$ Reactions with Linearly Polarized Photons", Atomic Data and Nuclear Data Tables $\underline{50,29(1992) .}$

A.C. Fonseca and D.R. Lehman, "NN P.Wave Effects in Polarized Deuteron Capture on Hydrogen: An Exact Faddeev Calculation", Few-Body Systems, Suppl. 6,279(1992).

H. Ito, W.W. Buck, and F. Gross, "Electromagnetic Properties of the Pion as a Composite Nambu Goldstone Boson", Phys. Rev. C45, 1918(1992).

L.C. Maximon, "Evaluation of the Spherical Bessel Transform of a Whittaker Function: An Application of a Difference Equation Method", J. Math. Phys. 33,2005(1992).

D.P. Heddle and L.C. Maximon, "LASPE: A Subroutine for Generating Straggling Distributions for Positrons and Electrons", Computer Physics Communications 70, $77(1992)$.

H. Haberzettl, "Cluster-Dynamical Approach to N-Body Scattering", Phys. Rev. C4ㅌ6,687(1992).

V. Punjabi, C.F. Perdrisat, E. Cheung, J. Yonnet, M. Boivin, E. Tomasi-Gustafsson, R. Siebert, R. Frascaria, E. Warde, S. Belostotsky, O. Miklucho, V. Sulimov, R. Abegg, and D.R. Lehman, " $\mathrm{T}_{20}$ in the Inclusive Breakup of $4.5 \mathrm{GeV}$ Polarized ${ }^{6} \mathrm{Li}$ ", Phys. Rev. C46 6 ,984(1992).

W.C. Parke, "Methods in Few-Body Nuclear Physics", Proceedings of the Hampton University Graduate Studies at the Continuous Electron Beam Accelerator Facility, May 29 through June 16, 1991, published by HUGS at CEBAF, Nuclear Physics Group, Department of Physics, Hampton University, Hampton Virginia, 1992, pp. 137-196.

H. Ito, W.W. Buck, and F. Gross, "The Axial Anomaly and the Dynamical Breaking of Chiral Symmetry in the $\gamma^{*} \pi^{0} \rightarrow \gamma$ reaction", Phys. Lett. B287,23(1992).

H. Haberzettl, "Relativistic Cluster Dynamics of Nucleons and Mesons. I. Kinematics and Covariance", Phys. Rev. C447,1237(1993).

Th. Januschke, T.N. Frank, H. Haberzettl, and W. Sandhas, "Neutron-Deuteron Scattering Calculations with Realistic NN Interactions using the W-matrix Representation of the Two-Body Input", Phys. Rev. C47,1401(1993). 
H. Ito and F. Gross, "Gauge Invariance and Compton Scattering from Relativistic Composite Systems", Phys. Rev. C뇨,1948(1993).

A.C. Fonseca and D.R. Lehman, "Full Three-Body Calculation for $\overrightarrow{\mathrm{d}}+\mathrm{p} \rightarrow{ }^{3} \mathrm{He}+\gamma$ with a Realistic NN Interaction", Phys. Rev. C4ㅛㅛ,R503(1993).

A.C. Fonseca, B.F. Gibson, and D.R. Lehman, "Approximate Ways to Treat the Jucleon-Nucleon Tensor Force in the Four-Nucleon Bound State", Phys. Rev. C48,2528(1993).

H. Ito and F. Gross, "Isoscalar Meson Exchange Currents and the Deuteron Form Factors", Phys. Rev. Lett. $11,2555(1993)$.

K. Gebhardt, W. Jäger, C. Jeitner, M. Vitz, E. Finckh, T.N. Frank, Th. Januschke, H. Haberzettl, and W. Sandhas, "Experimental and Theoretical Investigation of the ${ }^{2} \mathrm{H}(\mathrm{n}, \mathrm{nnp})$ Reaction and of the Neutron-Neutron Scattering Length" ${ }^{n}$, Nucl. Phys. A561,232(1993).

Xiaodong Li, L.E. Wright, and C. Bennhold, "Exclusive Quasifree Pion Photoproduction in the $\Delta$-Region", Phys. Rev. C488,816(1993).

L.C. Maximon, "High Energy Approximations for the Total Born Cross Section for Pair Production in a Screened Coulomb Field", Phys. Rev. C499,428(1994).

H. Haberzettl, "Relativistic Cluster Dynamics of Nucleons and Mesons. II. Formalism and Examples", Phys. Rev. C499,2142(1994).

H.R. Weller, R.M. Chasteler, B.S. Marks, R.G. Seyler, and D.R. Lehman, "Angular Distribution Coefficients for $(\gamma, \mathrm{X})$ Reactions with Circularly Polarized Photons and Polarized Targets and a Correction to Previous Tables", Atomic Data and Nuclear Data Tables $\underline{52}$, in press (1994):

A. Ramos and C. Bennhold, "The Nonmesonic Decay of Polarized Hypernuclei", Nucl. Phys. A, in press (1994)

Xiaodong Li, L.E. Wright, and C. Bennhold, "Exclusive Quasifree Radiative Pion Capture from Nuclei in the $\Delta$-Region", Phys. Rev. C, in press (1994).

L. Tiator, C. Bennhold, and S.S. Kamalov, "The $\eta$ NN Coupling Constant in Eta Photoproduction", Nucl. Phys. A, in press (1994).

B.F. Gibson, I.R. Afnan, J.A. Carlson, and D.R. Lehman, "Importance of BaryonBaryon Coupling in Hypernuclei", Prog. Theor. Phys. 92, in press (1994). 
PROGRESS

\section{PAPERS SUBMITTED}

T. Mart, C. Bennhold, C. Hyde-Wright, "Constraints on Coupling Constants in Charged $\Sigma$ Photoproduction", submitted to Phys. Rev. D.

S.K. Matthews, W.J. Briscoe, C. Bennhold, B.L. Berman, R.W. Caress, K.S. Dhuga,S.N. Dragic, N.J. Nicholas, M.F. Taragin, S.J. Greene, D.B. Barlow, B.M.K. Tefkens, C. Pillai, J.W. Price, L.D. Isenhower, M.E. Sadler, I. Slaus,I. Supek, L. Tiator, S.S. Kamalov, "Elastic Scattering of Pions from ${ }^{3} \mathrm{H}$ and ${ }^{3} \mathrm{He}$ into the Backward Hemisphere", submitted to Phys. Rev. C.

H. Haberzettl, "Covariant Off-Shell Model for Dynamical Quark Confinement", submitted to Nucl. Phys. A.

H. Haberzettl and W.C Parke, "Three-Nucleon Forces, Relativity, and Cluster Dynamics", submitted to Few-Body Systems.

H. Ito, "Octet of Goldstone Bosons and the Anomalous Magnetic Moments of Constituent Quarks", submitted to Phys. Lett. B.

H. Ito, " $G_{E}^{S}(q), G_{M}^{S}(q)$ and $G_{E}^{n}(q)$ in the Relativistic Quark Model of Nucleon with Meson Clouds", submitted to Phys. Rev. Lett.

W. W. Buck, R. A. Williams and H. Ito, "Elastic Charge Form Factors of $K$ Mesons", submitted to Phys. Lett. B.

D.R. Lehman, H.R. Weller, and R.M. Whitton, "Production of E1 Radiation in the ${ }^{2} \mathrm{H}(\mathrm{d}, \gamma){ }^{4} \mathrm{He}$ Reaction at Very Low Energies", submitted to Few-Body Systems.

\section{PAPERS IN PREPARATION}

D.R. Lehman and B.F. Gibson, "Formalism Underlying the $A=4 \Lambda$ Hypernuclei $1^{+}$ Equations", to be submitted to Phys. Rev. C.

Xiaodong Li, L.E. Wright, C. Bennhold, L. Tiator, "Quasifree Eta Photoproduction on Nuclei", to be submitted to Phys. Rev. C.

S.S. Kamalov, L. Tiator, and C. Bennhold, "Pion Scattering and Pion Photoproduction on the Deuteron", to be submitted to Phys. Rev. C.

A. Ramos and C. Bennhold, "Vector Meson Contributions to the Nonmesonic Hypernuclear Decay", to be submitted to Nucl. Phys. A.

H. Ito and M. Musolf, "Strange Axial-Vector Form Factors of Nucleon within the Relativistic Quark Model", to be submitted to Phys. Lett. B. 
PROGRESS

Y. Koike, D.R. Lehman, L.C. Maximon, and W.C. Parke, "A Possible Treatment of Three-Body Bound States with Long Range Interactions", to be submitted to Phys. Rer. C.

Y. Koike; D.R. Lehman, L.C. Maximon, and W.C. Parke, "Accurate Triton Calculation with a Local Realistic N-N Interaction with a Separable Expansion Method", to be submitted to Phys. Rev. C.

\section{INVITED TALKS AT CONFERENCES AND MEETINGS}

L.C. Maximon, "Quality of the Three-Nucleon Bound-State Wave Function from a Two-Nucleon Separable Expansion Method", invited contribution and talk, presented at the 13th International Conference on Few-Body Problems in Physics, Adelaide, Australia, 5-11 January 1992; associated abstract published with co-authors J. Haidenbauer, Y. Koike, D.R. Lehman, and W.C. Parke in Proceedings of the XIIIth International Conference on Few-Body Problems in Physics (Flinders University, Adelaide, Australia, 5-11 January 1992), edited by I.R. Afnan and R.T. Cahill, (Institute for Atomic Studies, Report FIAS-R-216, 1992) p. 302.

D.R. Lehman, "Faddeev Calculation of Polarization Observables in ${ }^{1} \mathrm{H}(\overrightarrow{\mathrm{d}}, \gamma)^{3} \mathrm{He}$, invited contribution and talk, presented at the 13th International Conference on FewBody Problems in Physics, Adelaide, Australia, 5-11 January 1992; associated abstract published with A.C. Fonseca in Proceedings of the XIIIth International Conference on Few-Body Problems in Physics (Flinders University, Adelaide, Australia, 5-11 January 1992), edited by I.R. Afnan and R.T. Cahill, (Institute for Atomic Studies, Report FLAS-R-216, 1992), p. 244.

D.R. Lehman, "NN P-Wave Effects in Polarized Deuteron Capture on Hydrogen: An Exact Faddeev Calculation", invited contribution and talk, presented at the 13th International Conference on Few-Body Problems in Physics, Adelaide, Australia, 5-11 January 1992; associated abstract published with A.C. Fonseca in Proceedings of the . XIIIth International Conference on Few-Body Problems in Physics (Flinders University, Adelaide, Australia, 5-11 January 1992), edited by I.R. Afnan and R.T. Cahill, (Institute for Atomic Studies, Report FIAS-R-216, 1992), p. 246.

H. Haberzettl, "The Efimov Effect in Neutron-Deuteron Scattering", presented at the Spring Meeting of the American Physical Society, Washington, DC, 23 April 1992, B.A.P.S. 37,2007(1992).

D.R. Lehman, "Few-Body Physics - Electromagnetic Interactions: An Overview (1990-92)", presented at the Gordon Research Conference on Photonuclear Reactions, (Tilton School), Tilton, New Hampshire, 10-14 August 1992.

C. Bennhold, "Photonuclear Physics with $\eta$-Mesons", presented at the 6th Workshop on Perspectives in Nuclear Physics at Intermediate Energies, Trieste, Italy, 3-8 May 1993, Trieste, Italy. 
C. Bennhold, "Polarization Observables in Eta Photoproduction", presented at the 1993 CAP/NSERC Summer Institute in Theoretical Physics, Queen's University, Kingston, Canada, 25-30 July 1993.

D.R. Lehman, "Continuum Faddeev Calculations and Electromagnetic Interactions: Dynamics of the Radiative Capture Reaction ${ }^{1} \mathrm{H}\left(\mathrm{d}_{\mathrm{pol}}, \gamma\right)^{3} \mathrm{He}$, presented at the Gordon Research Conference on Dynamics of Simple Systems in Chemistry and Physics, (Proctor Academy), Andover, New Hampshire, 16-20 August 1993.

H. Haberzettl, "Cluster-Dynamical Treatment of Three-Nucleon Forces", presented at the 14th European Conference on Few-Body Problems in Physics; associated paper published with W.C. Parke, in Proceedings of the XIVth European Conference on FewBody Problems in Physics (Amsterdam, The Netherlands, 23-27 August 1993), edited by B.L.G. Bakker and R. van Dantzig, Few-Body Systems Suppl. 7,274(1994).

C. Bennhold, "Polarization Observables in Pion Photoproduction on Few-Nucleon Systems", presented at the Workshop on (e,e'x) Experiments at the Saskatchewan Linear Accelerator, Saskatoon, Canada, 12-13 September 1993.

H. Haberzettl, "Relativistic Cluster Dynamics", Invited Lectures, presented at the CEBAF-INT Workshop on Relativity in Two- and Few-Body Nuclear Systems, CEBAF, Newport News, VA, 17-25 September 1993.

C. Bennhold, "Polarization Observables in Pion Scattering on the Trinucleon", presented at the Workshop on Meson-Nucleon Interactions and Few-Body Systems, Dubna, Russia, 29-30 April 1994.

C. Bennhold, "The Nonmesonic Weak Decay of Hypernuclei", presented at the International Conference on Mesons and Nuclei at Intermediate Energies, Dubna, Russia, 3-7 May 1994.

C. Bennhold, "Elastic Pion Scattering on Polarized ${ }^{3} \mathrm{He}$ ", presented at the International Conference on Mesons and Nuclei at Intermediate Energies, Dubna, Russia, 3-7 May 1994.

D.R. Lehman, "Capture of Polarized Deuterons on Protons", invited contribution and talk, presented at the 14th International Conference on Few-Body Problems in Physics, Williamsburg, VA, 26-31 May 1994; associated paper published with A.C. Fonseca in Contributed Papers Book for the 14th International IUPAP Conference on Few Body Problems in Physics (College of William and Mary, Williamsburg, VA, 26-31 May 1994). edited by Franz Gross, p. 50; to be published in the conference proceedings.

H. Ito, "Octet of Goldstone Bosons and the Electroweak Form Factors of Nucleon", invited contribution and talk, presented at the 14th International Conference on FewBody Problems in Physics, Williamsburg, VA, 26-31 May 1994; associated paper published in Contributed Papers Book for the 14th International IUPAP Conference on Few Body Problems in Physics (College of William and Mary, Williamsburg, VA, 26-31 May 1994), edited by Franz Gross, p. 401; to be published in the conference proceedings. 


\section{PROGRESS}

H. Haberzettl, "Relativistic Origin of Three-Nucleon Forces", invited contribution and talk, presented at the 14th International Conference on Few-Body Problems in Physics, Williamsburg, VA, 26-31 May 1994; associated paper published with W.C. Parke in Contributed Papers for the 14th International IUPAP Conference on Few-Body Problems in Physics (College of William and Mary, Williamsburg, VA, 26-31 May 1994), edited by Franz Gross, p. 881; to be published in the conference proceedings.

H. Haberzettl, "Relativistic Cluster Dynamics of Nucleons and Mesons", presented at the International Conference on Mesons and Nuclei at Intermediate Energies, Dubna, Russia, 3-7 May 1994: associated paper will appear in the Proceedings of the International Conference on Nucleons and Mesons at Intermediate Energies (Dubna, Russia, 2-7 May 1994), edited by M. Khankhasayev.

\section{INVITED TALKS AT UNIVERSITIES AND LABORATORIES}

C. Bennhold, "Eta Photoproduction from Nucleons and Nuclei", at the Dept. of Physics, Rensselaer Polytechnic Institute, Troy, NY, April 5, 1993.

C. Bennhold, "Photonuclear Reactions Involving $\eta$ Mesons", Nuclear Bag-Lunch Seminar, Department of Physics, The George Washington University, 23 April 1993.

C. Bennhold, "Photonuclear Physics with $\eta$-Mesons", at the Dept. of Physics, University of Barcelona, Barcelona, Spain, May 26, 1993.

C. Bennhold, "Photonuclear Physics with $\eta$-Mesons", at the Dept. of Physics, University of Richmond, VA, Oct. 15, 1993.

H. Haberzettl, "Relativistic Cluster Dynamics of Nucleons and Mesons", Department of Physics, University of Maryland, College Park, MD, 4 November 1991.

H. Haberzettl, "Relativistic Cluster Dynamics of Nucleons and Mesons", Theory Division, T-5, Los Alamos National Laboratory, Los Alamos, NM, 28 January 1992.

H. Haberzettl, "Cluster-dynamical Approach to the Scattering of Mesons and Nucleons", Theory Division, Argonne National Laboratory, Argonne, IL, 16 April 1992.

H. Haberzettl, "A Relativistic Scattering Theory for Nucleons and Mesons", Nuclear Bag-Lunch Seminar, Department of Physics, The George Washington University, 23 October 1992.

H. Haberzettl, "A Covariant Relativistic Scattering Theory for Composite Particles", Physikalisches Institut für Theoretische Physik II, Ruhr-Universität Bochum, Bochum, Germany, 22 June 1993.

H. Haberzettl, "A Covariant Relativistic Scattering Theory for Nucleons and Mesons", Institut für Theoretische Kernphysik, Universität Bonn, Bonn, Germany, 6 July 1993. 


\section{PROGRESS}

H. Haberzettl, "Dynamical Quark Confinement", Nuclear Bag-Lunch Seminar, Department of Physics, The George Washington University, 25 March 1994.

H. Ito, "Electromagnetic Gauge Invariance in Relativistic Few-Body Problems", at the Department of Physics, Carnegie-Mellon University, Pittsburgh, PA, 18 October 1991.

H. Ito, "Electromagnetic Gauge Invariance in Few-Body Problems", at the Department of Physics, University of Maryland, College Park, MD, 30 October 1991.

H. Ito, "SU(3) Flavor Symmetry in the Anomalous Magnetic Moments of Light Quarks", Nuclear Bag-Lunch Seminar, Department of Physics, The George Washington Uiniversity, 2 April 1993.

H. Ito, "Radiative Transition of Vector Mesons", University of Maryland, College Park, MD, Nuclear Theory Seminar, 13 May 1993.

D.R. Lehman, "Few-Body Physics - Electromagnetic Interactions: An Overview (199092)", Nuclear Bag-Lunch Seminar, Department of Physics, The George Washington University, 18 September 1992.

D.R. Lehman, "Few-Body Physics - Electromagnetic Interactions: An Overview (199092)", Experimental Nuclear Physics Seminar, Department of Physics, University of Maryland, College Park, MD, 12 October 1992.

D.R. Lehman, "Few-Body Physics - Electromagnetic Interactions: An Overview", Seminaro do Mestrado, Departamento de Fisica, Universidade de Lisboa, Lisboa, Portugal, 3 June 1993.

D.R. Lehman, "Radiative Capture of Polarized Deuterons on Hydrogen", Triangle Lniversities Nuclear Laboratory Seminar, Department of Physics, Duke University, Durham, NC, 17 June 1993.

D.R. Lehman, "Continuum Faddeev Calculations for Electromagnetic Processes", Triangle Universities Nuclear Theory Colloquium at Duke University, Department of Physics, Durham, NC, 22 February 1994.

W.C. Parke, "Lithium-6 Photodisintegration", Nuclear Bag-Lunch Seminar, Department of Physics, The George Washington University, 11 December 1992.

W.C. Parke, "Alpha-deuteron Radiative Capture", Triangle Universities Nuclear Laboratory Seminar, Department of Physics, Duke University, Durham, NC, 27 May 1993.

W.C. Parke, "Origin of the Elements", Invited talk, The George Washington University Chemistry Department, 8 April 1994. 
PROGRESS

\section{CONTRIBUTED PAPERS AND ABSTRACTS AT CONFERENCES}

H. Ito, "Dynamical Theory of the Compton Scattering from Relativistic Composite Systems". Annual Fall 'Meeting of the Division of Nuclear Phrsics of the American Physical Society, Santa Fe, NM, 14-17 October 1992, B.A.P.S. 3i.1317(1992).

H. Ito, "Quark Model of $\pi$ - and $\rho$-mesons and the $\rho \pi \gamma$-Exchange Current in the Deuteron Form Factors.", Annual Fall Meeting of the Division of Nuclear Physics of the American Physical Society, Santa Fe, NM, 14-17 October 1992, B.A.P.S. $\underline{37}, 1318(1992)$.

K. Gebhardt, W. Jäger, E. Finckh, T.N. Frank, Th. Januschke. W. Sandhas, and H. Haberzett1. "Determination of the Neutron-neutron Scattering Length $a_{n n}$ ", in the Proceedings of the XIIIth International Conference on Few-Bodr Problems in Physics (Flinders University, Adelaide, Australia, 5-11 January 1992), edited by I.R. Afnan and R.T. Cahill, (Institute for Atomic Studies, Report FIAS-R-216, 1992) p. 152.

T. N. Frank, H. Haberzettl, and W. Sandhas, "Neutron-deuteron Break-up Collisions, Proceedings of the XIIIth International Conference on Few-Bodr Problems in Physics (Flinders University, Adelaide, Australia, 5-11 January 1992), edited by I.R. Afnan and R.T. Cahill, (Institute for Atomic Studies, Report FIAS-R-216, 1992) p. 320.

K. Gebhardt, W. Jäger, E. Finckh, T.N. Frank, Th. Januschke. W. Sandhas, and H. Haberzettl, "Determination of the Neutron-neutron Scattering Length $a_{n n}$ ", in the Proceedings of the Spring Meeting of the Nuclear Phvsics Section of the German Physical Society (Salzburg, Austria, 24-28 February 1992), Verhandl. DPG (VI) 27, p. 121.

T.N. Frank, H. Haberzettl, and W. Sandhas (presenter), "Three-nucleon Break-up Processes", in the Proceedings of the XIIIth European Conference on Few-Body Physics (Marciana Marina, Isola d'Elba, Italy, 9-14 September 1991), edited by C. Ciofi degli Atti, E. Pace, G. Salmè, and S. Simula, (Few-Body Systems, Suppl. 6, 1992) p. 270.

H. Ito, W. W. Buck, and F. Gross, "The Axial Anomaly and the Dynamical Breaking of Chiral Symmetry in the $\gamma^{*} \pi^{b} \rightarrow \gamma$ reaction", in the Proceedings of the 2nd International Workshop on "Relativistic Aspects of Nuclear Physics", edited by $T$. Kodama et al., World Scientific, Singapore (1992), page 291.

V.B. Belyaev (presenter), M. Decker, H. Haberzettl, L.J. Khaskilevitch, and W. Sandhas, "Muonic Molecules of Light Nuclei", in the Proceedings of the XIIIth European Conference on Few-Body Physics (Marciana Marina, Isola d'Elba, Italy, 9-14 September 1991), edited by C. Ciofi degli Atti, E. Pace, G. Salmè, and S. Simula, (FewBody Systems, $\underline{\text { Suppl. } 6}$, 1992) p. 332.

C. Bennhold, L. Tiator, and S.S. Kamalov, "Photonuclear Physics with Eta-Mesons" in the Proceedings of the 6th Workshop on Perspectives in Nuclear Physics at Intermediate Energies, Trieste, Italy, 3-8 May 1993, (in press). 
L. Tiator, S.S. Kamalov, and C. Bennhold, "Polarization Observables in Pion Photoproduction on Light Nuclei", in the Proceedings of the 6th Workshop on Perspectives in Duclear Physics at Intermediate Energies, Trieste, Italy, 3-8 May 1993, (in press).

A. Ramos, C. Bennhold, J. Nieves, and E. Oset, "Weak Decay of Hypernuclei", in the Proceedings of the 13th Particles and Nuclei International Conference (PANIC XIII), Perugia, Italy, 28 June - 2 July 1993, (in press).

A. Parreno, A. Ramos, C. Bennhold, and D. Halderson, "Short-Range Correlations in the Weak Decay of Hypernuclei", in the Proceedings of the International Workshop on Drnamical Features of Nuclei and Finite Fermi Systems, Sitges (Barcelona), Spain, 1317 September 1993, (in press).

C. Bennhold and A. Ramos, "The Nonmesonic Hypernuclear Decay in a Weak MesonExchange Model", in the Proceedings of the 5th Conference on the Intersections of Particle and Nuclear Physics, St. Petersburg, Florida, 31 May - 6 June 1994, (in press).

M. Espy, D. Dehnhard, D. Davis, C. Edwards, J. Langenbrunner, J.M. O'Donnell, M.Palarczyk, G. Burleson, S. Blanchard, W.R. Gibbs, B. Lail, B. Nelson, B. Park, Q. Zhao, O. Hausser, W. Cummings, P. Delheij, W. Lorenzen, R. Henderson, D.Thiessen, B.K. Jennings, E. Brash, M. Jones, B. Larson, B. Brinkmoeller, K. Maeda, C.L. Morris, S. Pentilla, D. Swenson, D. Tupa, C. Bennhold, L. Tiator, and S.S.Kamalov, "Elastic $\pi^{+}$Scattering on Polarized ${ }^{3} \mathrm{He}$ at $\Delta$ Resonance Energies", in the Proceedings of the International Conference on Mesons and Nuclei at Intermediate Energies, Dubna, Russia, 3-7 May 1994, (in press).

C. Bennhold and A. Ramos, "The Nonmesonic Weak Decay of Hypernuclei", in the Proceedings of the International Conference on Mesons and Nuclei at Intermediate Energies, Dubna, Russia, 3-7 May 1994, (in press).

L. Tiator, C. Bennhold, and S.S. Kamalov, "Born and Resonance Couplings in $\eta$ and $\eta$ ' Photoproduction", in the Proceedings of the International Conference on Mesons and Nuclei at Intermediate Energies, Dubna, Russia, 3-7 May 1994, (in press).

S.K. Matthews, W.J. Briscoe, C. Bennhold, B.L. Berman, R.W. Caress, K.S. Dhuga, S.N. Dragic, N.J. Nicholas, M.F. Taragin, S.J. Greene, D.B. Barlow, B.M.K. Nefkens, C. Pillai, J.W. Price, L.D. Isenhower, M.E. Sadler, I. Slaus, and I. Supek, "Elastic Scattering of Pions from ${ }^{3} \mathrm{H}$ and ${ }^{3} \mathrm{He}$ into the Backward Hemisphere", in the Proceedings of the 14th International Conference on Few-Body Problems in Physics, Williamsburg, VA, 26-31 May 1994, (in press).

T. Mart and C. Bennhold, "Suppression of Coupling Constants in $\Sigma$ Photoproduction", in the Proceedings of the International Conference on Hypernuclear and Strange Particle Physics, Vancouver, Canada, 4-8 July 1994, (in press).

C. Bennhold, L. Tiator, and S.S. Kamalov, "Strangeness Content of the Nucleon and the $\eta N N$ Coupling", in the Proceedings of the International Conference on Hvpernuclear and Strange Particle Physics, Vancouver, Canada, July 4-8, 1994 (in press) 
Y. Koike, D.R. Lehman, L.C. Maximon, and W.C. Parke, "A Possible Treatment of Three-Body Bound States with Long Range Interaction", poster, in Contributed Papers Book for the 14th International IUPAP Conference on Few Body Problems in Physics (Collëge of William and Mary, Williamsburg, VA, 26-31 May 1994), edited by Franz Gross, p. 800.

Y. Koike, D.R. Lehman, L.C. Maximon, and W.C. Parke, "Accurate Triton Calculation with a Local Realistic N-N Interaction with a Separable Expansion Method", invited contribution and talk (given by Y. Koike), in Contributed Papers Book for the 14th International IUPAP Conference on Few Body Problems in Physics (College of William and Mary, Williamsburg, VA, 26-31 May 1994), edited by Franz Gross, p. 804, to be published in the proceedings.

\section{VISITORS TO THE GW NUCLEAR THEORY GROUP}

Yasuro Koike

Department of Physics

Hosei University (Tokyo)

18 August 1991

Thomas D. Cohen

Department of Physics

University of Maryland

20 September 1991

Carl Werntz

Department of Physics

Catholic University

4 October 1991

George Rupp

Department of Physics

University of Maryland and

I.N.I.C. (University of Lisbon)

Lisbon, Portugal

1 November 1991

Wayne N. Polyzou

Department of Physics

Eniversity of Iowa

30-31 January 1992
Antonio C. Fonseca

I.N.I.C. (University of Lisbon)

Lisbon, Portugal

24-31 August 1991

19-25 April 1992

Manoj K. Banerjee

Department of Physics

University of Maryland

27 September 1991

Abraham Rinat

Weizmann Institute

Rehovoth, Israel

7 October 1991

Antonio Delfino

Department of Physics

University of Maryland and

Instituto de Física

Universidade Federal Fluminense

Rio de Janeiro, Brasil

22 November 1991

Carl M. Shakin

Department of Physics

Brooklyn College

13 March 1992 
PROGRESS

James J. Griffin

Department of Physics

University of Maryland

3 April 1992

Yasuro Koike

Department of Physics

Hosei University (Tokyo)

3-10 September 1992

T.-S. H. Lee

Physics Division

Argonne National Laboratory

13 November 1992

Antonio C. Fonseca

I.N.I.C. (University of Lisbon)

Lisbon, Portugal

Visited with D.R. Lehman at

TUNL (Duke University)

1 February 1993 to 16 March 1993

Sabit S. Kamalov

Joint Institute of Nuclear Research

Dubna, Russia

3-29 July 1993

Yasuro Koike

Physics Department

Hosei University

Chiyoda, Tokyo 102, Japan

12 July $1993-8$ April 1994

1 June 1994 - 4 June 1994

Stephen J. Wallace

Department of Physics

Eniversity of Maryland

College Park, MD 20742

22 October 1993

Elizabeth J. Beise

Department of Physics

University of Maryland

College Park, MD 20742

19 November 1993
R.A. Eramzhyan

Joint Institute for Nuclear Research

Dubna, Russia

24-25 September 1992

Mark Bolsterli

Physics Division

National Science Foundation

16 April 1993
Lothar Tiator

Institut für Kernphysik

Universität Mainz

D 55099 Mainz, Germany

14-21 July 1993

John A. Tjon

Institute for Theoretical Physics

University of Utrecht

3508 TA Utrecht, The Netherlands

8-9 October 1993

Johan J. deSwart

Institute for Theoretical Physics

University of Nijmegen

Nijmegen, The Netherlands

4-6 November 1993

John Michael Finn

Physics Department

College of William and Mary

Williamsburg, VA 23185

3 December 1993 
Xiaodong Li

Department of Physics

Ohio University

Athens, $\mathrm{OH} 45701$

4-9 December 1993

Bradley D. Keister

Department of Physics

Carnegie-Mellon University

Pittsburgh, PA 15213 and

Physics Division

National Science Foundation

4201 Wilson Boulevard

Arlington, VA 22230

4 February 1994

Michael J. Musolf

Department of Physics

Old Dominion University

Norfolk, VA 23529

and

Theory Group, Physics Division

CEBAF

12000 Jefferson Avenue

Newport News, VA 23606

4-5 March 1994

Daniel R. Phillips

School of Physical Science

Flinders University of South Australia GPO Box 2100

Adelaide 5001, Australia

15-20 May 1994

Iraj R. Afnan

School of Physical Science

Flinders University of South Australia Faculty of Science and Technology

GPO Box 2100

Adelaide 5001, Australia

24 May 1994

Ana Eiro

Centro de Fisica Nuclear

Univ de Lisboa

Av Gama Pinto 2

1699 Lisboa Codex, Portugal

1-8 June 1994
Zein-Eddine Meziani

Department of Physics

Barton Hall A323

Temple University

Philadelphia, PA 19123

14-15 January 1994

Rocco Schiavilla

Department of Physics

Old Dominion University

Norfolk, VA 23529

and

Theory Group, Physics Division

CEBAF

12000 Jefferson Avenue

Newport News. VA 23606

18-19 February 1994

Benjamin F. Gibson

T-5 MS-283

Los Alamos National Laboratory

Los Alamos, New Mexico 87545

20-21 April 1994
Werner Sandhas

Physikalisches Institut der
Universität Bonn

Endenicher Allee 11-13

533115 Bonn, Germany

22-24 May 1994

Sinsho Oryu

Department of Physics

Science University of Tokyo

Noda, Chiba 278, Japan

2 June 1994

Terry Mart

Institut für Kernphysik

Universität Mainz

D 55099 Mainz, Germany

6 June - 3 July 1994 
PROGRESS

\section{RESEARCH PROGRESS BY TOPIC}

In order to make it easy for the reader to see the specific research carried out and the progress made, the following report of progress is done by topic. Each item has a format layout of Topic, Investigators, Objective, Significance, and Description of Progress, followed at the end by the relevant references. As is clear from the topics listed, the emphasis of the GW nuclear theory group has been on the structure and electromagnetic interactions of few-body nuclei. Both low- and intermediate-energy electromagnetic disintegration of these nuclei is considered, including coherent photoproduction of $\pi$ mesons. When the excitation energy of the target nucleus is low, the aim has been to handle the continuum part of the theoretical work numerically with no approximations, that is, by means of full three- or four-body dynamics. When structure questions are the issue, numerically accurate calculations are always carried through, limited only by the underlying two-body or three-body interactions used as input. Implicit in our work is the question of how far one can go within the traditional nuclear physics framework, i.e., nucleons and mesons in a nonrelativistic setting. Our central goal is to carry through state-of-the-art few-body calculations that will serve as a means of determining at what point standard nuclear physics requires introduction of relativity and/or quark degrees of freedom in order to understand the phenomena in question. So far, the problems considered were mostly concerned with low- to mediumenergy regimes where little evidence was found that requires going beyond the traditional approach. This situation is very likely to change in the years to come as we become significantly involved in CEBAF-type intermediate-energy physics. In anticipation of these changes, efforts have been directed towards including relativity in a consistent manner into the dynamical framework of few-body physics. At present, many of the structural and mathematical questions associated with a covariant formulation of the relativistic many-body scattering problem are being clarified, thus setting the stage for realistic applications in the future.

A significant guiding factor in the work of the GW theory group is the current experimental effort and capabilities in the areas of interest. The work at low excitation energies is motivated by the beautiful radiative capture experiments with polarized beams being carried out at TUNL, Wisconsin, IUCF, and SIN. At intermediate energies, the capabilities of coincidence experiments at NIKHEF has been a source of inspiration for carrying out detailed three-body calculations. More recently, the existence of a source of polarized gammas at the LEGS facility (Brookhaven) has lead us to examine what can be learned about the D-state of ${ }^{4} \mathrm{He}$ by disintegration into two deuterons with linearly polarized gammas. At the same time, much of the impressive data that has already been obtained at the new higher-duty accelerators, and data which will be forthcoming at the new facilities like CEBAF, depend critically on a full understanding of the QED processes inherent to electron scattering, i.e., the ability to calculate and account accurately for these effects: One member of our group (LCM) contributes significantly in this area as is evident from his putting together of a manual for the CEBAF experimentalists on radiative corrections. Finally, all of us interact and collaborate with the GW nuclear experimentalists. In fact, the interactions between the GW nuclear theorists and experimentalists has been on the increase. This tradition has been further augmented by the addition of Cornelius Bennhold to our group. He has been working closely with both Professors Briscoe and Berman on the interpretation of their LAMPF pion scattering experiments. 
PROGRESS

TOPIC: Photo- and Electroproduction of $\eta$-Mesons on the Nucleon

INVESTIGATORS: C. Bennhold, L. Tiator (U. of Mainz, Germany), S.S. Kamalov (JINR, Dubna. Russia), and R. Pratt (Ph.D. Student)

OBJECTIVE: To develop an operator for $\eta$ electromagnetic production on the nucleon in the excitation region of the $S_{11}(1535)$ resonance and extract the $\eta N N$ coupling constant.

SIGNIFICANCE: In contrast to pions which excite the $\Delta$ (with isospin $T=3 / 2$ ) as well as $\mathrm{N}^{*}(\mathrm{~T}=1 / 2)$ resonances simultaneously, the $\eta$-meson, due to its isoscalar nature, can be employed to selectively probe $\mathrm{N}^{*}$ states. In the low-energy regime there is significant preference for intermediate excitation of the $S_{11}(1535)$ state in the $\eta \mathrm{N}$ system which has not yet been understood in quark models. A successful description of the resonance sector in the $\gamma \mathrm{N} \rightarrow \eta \mathrm{N}$ reaction should permit the extraction of the $\eta \mathrm{NN}$ coupling constant which via $\eta-\eta^{\prime}$ mixing is related to the $\mathrm{U}_{A}(1)$ anomaly of QCD and the axial flavor singlet coupling measured by recent EMC data.

DESCRIPTION OF PROGRESS: The initial phase of this project focused on the description of the resonance sector of the eta photoproduction process. However, very recent data taken in Bonn ${ }^{2}$ (ELSA) and Mainz ${ }^{3}$ (MAMI B) which are currently being analyzed require an eta production mechanism that goes beyond mere resonance production. We have therefore included ${ }^{4}$ background contributions such as the s- and $\mathrm{u}$-channel nucleon Born terms and vector meson $\mathrm{t}$-channel exchanges. The resonance sector was constrained via an isobar model that used independent hadronic and electromagnetic reactions to determine the couplings to the resonances. The major remaining uncertainty comes from the structure of the $\eta \mathrm{NN}$-vertex. In contrast to the $\pi \mathrm{N}$-interaction, little is known about the $\eta \mathrm{N}$-interaction and, consequently, about the $\eta \mathrm{NN}$-vertex. Since the eta mass is so much larger than the pion mass - leading to large SU(3) x SU(3) symmetry breaking - there is no compelling reason to select pseudovector (PV) rather than pseudoscalar (PS) coupling for the $\eta N N$-vertex. The uncertainty in the structure of the $\eta \mathrm{NN}$-vertex is equally large regarding the magnitude of the coupling constant. The SU(3) value of $g_{\eta}^{2} / 4 \pi$ is related to the $\pi N$ constant and is in the range of $0.8-1.7$, while typical values obtained in fits with the OBEP NN potential can lie anywhere between $3-7$.

Since in our model the resonance sector is well constrained by other related but independent reactions, we use the $(\gamma, \eta)$ data to extract information on the $\eta N N$-vertex. While the total $(\gamma, \eta)$ cross section on the proton can be well reproduced by either a small coupling constant with PS-coupling or a large value with PV-form, the angular distribution singles out the small constant. Furthermore, we have undertaken a systematic study ${ }^{5}$ of all single and double polarization observables of the $(\gamma, \eta)$ reaction and found that especially the recoil polarization is very sensitive to the PS-PV difference. On the other hand, the photon asymmetry $\Sigma$ is almost determined by the $D_{13}(1520)$ resonance which barely shows up in the differential cross section. These sensitivities can be understood by expressing the polarization observables in terms of the relevant multipoles; in most cases it is the dominant $E_{0}+$ that interferes with a smaller multipole and generates the observed sensitivity. We are currently studying a complete set of polarization observables in $\left(e, e^{\prime} \eta\right)$ where - in contrast to $(\gamma, \eta)$ with 15 
PROGRESS

polarization observables - one has 32 single, double and even triple polarization observables.

${ }^{1}$ C. Bennhold and H. Tanabe, Nucl. Phys. A $\underline{50}, 625(1991)$; Phys. Lett. B $\underline{43}, 13(1990)$; Lect. Notes in Phys. 365,190(1990).

${ }^{2}$ G. Anton, Proc. 6th Workshop on Persp. in Nucl. Phys. at Intermed. Energies, Trieste, Italy, May 3-8, 1993 (in press).

${ }^{3} \mathrm{~B}$. Krusche, as in Ref. 2.

${ }^{4}$ C. Bennhold, et. al., as in Ref. 2.

${ }^{5}$ L. Tiator, C. Bennhold, and S.S. Kamalov, Nucl. Phys. A (in press). 
PROGRESS

TOPIC: Exclusive Quasifree Pion Photoproduction and Radiative Pion Capture

INVESTIGATORS: Xiaodong Li (Ohio U.), L.E. Wright (Ohio U.), and C. Bennhold

OBJECTIVE: To provide a calculational framework for the reactions $(\gamma, \pi \mathrm{N})$ and $(\pi, \gamma N)$ in order to extract $\Delta$ properties modified by the nucleus.

SIGNIFICANCE: In the last decade, much has been deduced about the $\Delta$-nucleus interaction indirectly by studying pion-nucleus scattering and photoproduction. The new high-duty cycle accelerators allow for measuring direct $\Delta$-knockout in $(\gamma, \Delta)$ and $\left(e, e^{\prime} \Delta\right)$ reactions where the delta is detected as a $\pi \mathrm{N}$-system in coincidence. Providing a rigorous calculational framework for these processes (which has not been done until now) should at last permit the extraction of $\Delta$ properties - modified by the nuclear medium - directly. Furthermore, since pion radiative capture and pion photoproduction are related via time reversal invariance, the same calculational framework should describe both sets of experiments.

DESCRIPTION OF PROGRESS: The exclusive A $(\gamma, \pi \mathrm{N}) \mathrm{B}$ reaction offers an ideal laboratory for studying the $\Delta$ resonance in the nuclear medium. It allows for more direct access to the $\Delta$ than the reaction $\mathrm{A}(\gamma, \pi) \mathrm{B}$ since the final nucleon is no longer bound and the sensitivity to the nuclear structure of the target is thereby greatly reduced. In the past, information on the $\Delta$-nucleus interaction could only be extracted indirectly from reactions like pion elastic and inelastic scattering, and pion photoproduction on nuclei.

In quasifree pion photoproduction the only information required for the target is the single-particle bound wave function and the spectroscopic factor, which is only an overall factor in the cross section. Kinematically, the reaction provides a great deal of flexibility since the target can take up a wide range of momentum transfer and for finite nuclei, little energy. We propose a kinematic arrangement that can best expose the information from the production vertex by fixing the lengths of each momentum vector in the overall momentum conservation. Encouraged by the success of our previous DWIA approach in the analysis of the $A(\gamma, \pi) B$ reaction, our goal is to set up a nonlocal DWIA formalism for the $\mathrm{A}(\gamma, \pi \mathrm{N}) \mathrm{B}$ reaction using the same standard physics inputs. In this context, the term nonlocal refers to an exact treatment of all momentum dependencies in the elementary operator and the optical potentials. Even though previous calculations on $\mathrm{A}(\gamma, \pi) \mathrm{B}$ and inclusive quasifree $\mathrm{A}(\gamma, \pi) \mathrm{X}$ reactions were all performed in a nonlocal framework, no nonlocal computations have been performed for exclusive $\mathrm{A}(\gamma, \pi \mathrm{N}) \mathrm{B}$ processes. The theoretical results available up to now employ a factorized impulse approximation. Following the analogous development of $A(\gamma, \pi) B$ calculations we find it important to assess the significance of nonlocal effects. The additional nucleon in the final state adds new computational challenges because additional partial waves need to be summed over.

At the present stage ${ }^{3}$, we neglect possible medium modifications and only use the free production amplitudes to see if there are significant deviations of experiments from our nonlocal DWIA description. As far as the local DWIA calculation is concerned, the distortions from final state interactions always attenuate the PWIA cross sections. Although distortion plays an important role in getting the correct magnitudes, it does 
PROGRESS

little to change the shape of the curves. Nonlocal effects always enhance the local DWIA cross sections and in some cases they are quite significant. In general, quantitative comparison of theory and experiment requires a nonlocal calculation. We find that we can properly reproduce $\left(\gamma, \pi^{-} \mathrm{p}\right)$ data from Tomsk while we overpredict the recent BATES data by up to a factor of three. Using time-reversal invariance we have exiended our formalism ${ }^{5}$ to simultaneously study the exclusive quasifree radiative capture of pions. Here we find good agreement with recently measured $\left(\pi^{+}, \gamma \mathrm{p}\right)$ TPIUMF data on ${ }^{16} \mathrm{O}$ in the same kinematic region as that the BATES experiment, suggesting the possibility that the BATES data are flawed. A new experiment that will reexamine the BATES measurements has recently been approved at NIKHEF ${ }^{6}$. We also find that the photon asymmetry is a very good observable to complement the cross section measurements. It comes mainly from the $\Delta$ resonance, is free from normalization problems, is predicted to be large, and is relatively insensitive to the distortions and nonlocal effects. An experiment at LEGS (BNL) has already been approved to verify our findings ${ }^{7}$.

${ }^{1}$ L. Tiator and L. E. Wright, Phys. Rev. C $\underline{30}, 989(1984)$; C. Bennhold, L. Tiator and L.E. Wright, Can. J. Phys. $68,1270(1990)$.

${ }^{2}$ J.M. Laget, Nucl. Phys. A194,81(1972); I.V. Glavanakov; Sov. J. Nucl. Phys. $3 \underline{52}, 205(1990)$.

${ }^{3}$ Xiaodong Li, L.E. Wright and C. Bennhold, Phys. Rev. C48,816(1993).

${ }_{5}^{4}$ L.D. Pham et al., Phys. Rev. C46,621(1992).

${ }^{5}$ Xiaodong Li, L.E. Wright, C. Bennhold, Phys. Rev. C (in press).

${ }_{6}^{6} \mathrm{G}$. van der Steenhoven, private communication.

$' \mathrm{~h}$. Hicks, private communication. 
PROGRESS

TOPIC: The Nonmesonic Weak Decay of Hypernuclei

INVESTIGATORS: A. Ramos (U. of Barcelona, Spain), C. Bennhold, and A. Parreno (Ph.D. student at U. of Barcelona, Spain)

OBJECTIVE: To extract information on the weak hadronic vertices by analyzing the nonmesonic hypernuclear decay.

SIGNIFICANCE: The weak decay of the lambda and other byperons is of interest since it involves a weak interaction process without leptons. The mesonic decay of the free $\Lambda$ hyperon, $\Lambda \rightarrow N \pi$, dominates the leptonic one, $\Lambda \rightarrow p+e^{-}+\nu_{e}$, by more than a factor of 1000 due to the three-body final state in the latter decay mode. Hypernuclei in their ground states also decay via weak interaction mechanisms when they are stable against strong decay modes such as particle emission. However, the final nucleon produced in the mesonic decay of a $\Lambda$ has a very low momentum $(<100 \mathrm{MeV} / \mathrm{c})$ and is therefore Pauli blocked in the nuclear medium. Thus, the mesonic mode is strongly suppressed inside all but the lightest hypernuclei. It was recognized early that the dominant decay for heavier hypernuclei would be due to the two-body, nonmesonic mode $\Lambda N \rightarrow N N$, where the pion may now be viewed as being virtual and absorbed on a second nucleon bound in the nucleus. This process can also receive contributions from more massive mesons whose production threshold is too high for the free-space $\Lambda$ decay.

The nonmesonic decay mode $\Lambda N \rightarrow N N$ will complement the information available from the weak $\mathrm{NN}$-interaction, which is accessible experimentally through parity-violating $\mathrm{NN}$-scattering. The physics of the $\Lambda \mathrm{N} \rightarrow \mathrm{NN}$ decay may be richer since it involves weak strangeness-changing $(\Delta S=1)$ interactions. Furthermore, both the parity-conserving (PC) and parity-violating (PV) partial rates can be measured, whereas the strong force masks the PC interaction of the weak NN interaction.

DESCRIPTION OF PROGRESS: This study was motivated by new experimental results from Brookhaven and KEK in Japan. New measurements of total and partial hypernuclear decay rates for ${ }^{5} \mathrm{He}_{\mathrm{A}}$ and ${ }^{12} \mathrm{C}_{\mathrm{A}}$ with reduced experimental uncertainties have been obtained at Brookhaven. A recently completed experiment at $\mathrm{KEK}^{1}$ measured the asymmetry of the angular distribution of protons coming from the decay of polarized hypernuclei produced via the $\left(\pi^{+}, \mathrm{K}^{+}\right)$reaction. Most theoretical studies, reviewed recently in Ref. 2, have analyzed the nonmesonic decay mode in nuclear matter, assuming the lambda to be at rest and keeping only the relative $\Lambda \mathrm{N}$ s-wave contributions. Over the last several years, we have developed a formalism ${ }^{3}$ for relativistic calculations of the nonmesonic hypernuclear decay in a shell-model framework. Since we study polarization observables involving the ejected nucleons, we have chosen the Dirac phenomenology approach which has successfully predicted spin observables in elastic proton-nucleus scattering: In order to minimize the model dependency, the details of the nuclear structure input are treated as well as possible. In this spirit, we avoid the transformation into the relative $\Lambda \mathrm{N}$ two-body frame and can therefore easily incorporate nucleon orbitals with arbitrary $\mathrm{n}, \mathrm{l}$, and $\mathrm{j}$. Final-state distortions of the outgoing nucleons with the residual nuclear state were generated using a relativistic optical potential. The nucleon and lambda bound-state wave functions are solutions of the Dirac equation with scalar, vector and tensor potentials which were adjusted to reproduce binding energies and charge form factors. Appropriate 
PROGRESS

spectroscopic factors, corrected for center-of-mass (c.m.) motion, are employed to properly include the shell structure of the hypernucleus under study. Short-range correlations (SRC) are accounted for by using a $\Lambda N$ correlation function that is based on the Nijmegen YN-potential. Special attention has to be paid ${ }^{4}$ to the effect of SRC in a relativistic two-body matrix element, since including SRC straightforwardly leads to a suppression of the matrix elements that is larger by about a factor of two compared to what is obtained in standard nonrelativistic calculations, where the correlation function is introduced after a nonrelativistic reduction has been performed. It can be shown that a correlated potential of the nonrelativistic type can be obtained from the relativistic Feynman amplitude if pseudovector (PV) coupling is assumed for both vertices.

Previous calculations have mostly employed a one-pion exchange mechanism to describe the reaction $\Lambda N \rightarrow N N$ which has then commonly been evaluated in nuclear matter. We are exploring ${ }^{5}$ the role of heavier meson exchanges, $\eta, K, \rho, \omega$, and $\mathrm{K}^{*}$, thus constructing a complete weak one-boson exchange potential. The strong couplings are taken from either the Nijmegen or Juelich B interaction while the weak vertices are computed using a quark model and $\mathrm{SU}(6)_{w}$ symmetry $^{6}$. For example, the parityconserving weak vertices are computed by using a pole model that requires the weak meson $\rightarrow$ meson and baryon $\rightarrow$ baryon transition amplitudes as input. Applying $\mathrm{SU}(6)_{w}$ symmetry and enforcing the $\Delta \mathrm{I}=1 / 2$ rule yields the necessary meson $\rightarrow$ meson amplitudes in terms of the $\mathrm{K} \rightarrow \pi$ amplitude. Employing PCAC, this amplitude can be related to the physical $\mathrm{K} \rightarrow \pi \pi$ decay rate. Similarly, the baryon $\rightarrow$ baryon amplitudes can be related to the physical free $\Lambda$ and $\Sigma$ mesonic decay rates.

We find that without form factors and SRCs the vector mesons give a large contribution. However, including the short range behavior properly leads to a considerable suppression. In fact, the interferences between the different mesons conspire to give a decay rate that is only $20-30 \%$ larger than the pion exchange only. Our results for the total decay rates are in agreement with experimental data, while the measured ratio $\Gamma_{n} / \Gamma_{p}$ - with very large error bars - is underestimated by more than a factor of two. The calculated asymmetries, on the other hand, are in reasonable agreement with the measurements once the hypernuclear polarization obtained via the $\left(\pi^{+}, \mathrm{K}^{+}\right)$reaction has been corrected for hypernuclear structure and finite detector resolution effects.

${ }^{1}$ S. Ajimura et al., Phys. Lett. B282,293(1992).

${ }^{2} \mathrm{~J}$. Cohen, Prog. Part. Nucl. Phys. 25,139(1990).

${ }^{3}$ A. Ramos, C. Bennhold, E. van Meijgaard, and B.K. Jennings, Phys. Lett. B264,233(1991); C. Bennhold and A. Ramos, Phys. Rev. C45,3017(1992); A. Ramos, E. van Meijgaard, C. Bennhold, and B.K. Jennings, Nucl. Phys. A 544,703(1992); A. Ramos, C. Bennhold, E. van Meijgaard, and B.K. Jennings, Nucl. Phys. A $\underline{547}, 103 \mathrm{c}(1992)$.

4 A. Parreno, A. Ramos, C. Bennhold, D. Halderson, Proceedings of the International Workshop on Dynamical Features of Nuclei and Finite Fermi Systems, Sitges (Barcelona), Spain, Sept. 13-17, 1993 (in press).

A. Ramos and C. Bennhold, Nucl. Phys. A (in press); A. Ramos, C. Bennhold, J. Nieves, and E. Oset, Proceedings of the 13th Particles and Nuclei International Conference (PANIC XIII), Perugia, Italy, June 28 - July 2, 1993 (in press); C. Bennhold and A. Ramos, Proceedings of the 5th Conference on the Intersections of 
Particle and Nuclear Physics, St. Petersburg, Florida, May 31-June 6, 1994, (in press); C. Bennhold and A. Ramos, Proceedings of the International Conference on Mesons and Nuclei at Intermediate Energies, Dubna, Russia, May 3-7, 1994, (in press).

${ }^{\circ} \mathrm{L}$. de la Torre, Ph.D thesis, Univ. of Massachusetts, 1982. 
PROGRESS

TOPIC: Pion- and Photon-Induced Reactions on the Trinucleon

INVESTIGATORS: S.S. Kamalov (JINR, Dubna, Russia), C. Bennhold, and L. Tiator (U. of Mainz, Germany)

OBJECTIVE: To explore the limits of the multiple scattering formalism and the Impulse Approximation by describing all available data for pion scattering, single charge exchange pion photoproduction and eta production on the trinucleon in a unified coupled-channels framework.

SIGNIFICANCE: Pion- and photon-induced reactions on the trinucleon are an ideal testing ground to investigate the interaction of mesons and photons with nuclei and search for possible modifications of resonance properties in the nuclear medium. Nuclear structure uncertainties in the trinucleon wave function are under control since correlated three-body amplitudes can be obtained by solving the Faddeev equations with realistic nucleon-nucleon potentials. This is in contrast to reactions on p-shell or heavier nuclei where single particle wave functions are computed in the shell model while nuclear structure coefficients are constrained by beta-decay rates, electromagnetic form factors and other observables. Ambiguities remain, especially regarding the magnitude of the spin-flip matrix elements. The trinucleon is therefore well suited as a nuclear target to examine whether reactions like pion scattering, pion single charge exchange, pion-induced $\eta$ production as well as pion and eta photoproduction can all be described in one consistent framework, using only the elementary $\pi \mathrm{N} \rightarrow \pi \mathrm{N}, \pi \mathrm{N} \rightarrow \eta \mathrm{N}$, $\gamma \mathrm{N} \rightarrow \pi \mathrm{N}$ and $\gamma \mathrm{N} \rightarrow \eta \mathrm{N}$ amplitudes as input.

DESCRIPTION OF PROGRESS: In our coupled-channels formalism ${ }^{1}$ the pion elastic scattering data on ${ }^{3} \mathrm{He}$ and ${ }^{3} \mathrm{H}$ were well reproduced in almost the entire kinematical region considered. Only for pion kinetic energies above $180 \mathrm{MeV}$ and backward pion angles - a region with few experimental data - hints of an inadequate description appear. The asymmetries are large in contrast to the asymmetries measured on p-shell nuclei. Noteworthy is the change of $\operatorname{sign}^{2}$ in $A_{y}$ from +1 to -1 when one moves into the $\Delta$ resonance region. This effect - caused by pion multiple scattering - has recently been verified at LAMPF at $256 \mathrm{MeV}$. However, the preliminary data at $180 \mathrm{MeV}$ show large deviations from the theoretical predications ${ }^{3}$. Second-order optical potential effects may be responsible for these deviations. At $T_{\pi}=500 \mathrm{MeV}$ and in the forward direction, $A_{y}$ is entirely determined by the $D_{13}(1520)$ resonance contribution.

In contrast to pion elastic scattering, the pion single charge exchange calculations agree with the data only up to $200 \mathrm{MeV}$; at higher energies the theoretical description dramatically fails to explain the measurements by underestimating them up to two orders of magnitude. Since the $\pi \mathrm{N}$ amplitudes are presumably well known, this discrepancy may be an indication of two- and three-body processes that go beyond the impulse approximation. A similar phenomenon has been observed in pion photoproduction on ${ }^{3} \mathrm{He}$ at large momentum transfer ${ }^{4}$. Overall, we found - after properly including pion final-state interaction (FSI) with the important two-step process ${ }^{3} \mathrm{He}\left(\gamma, \pi^{\circ}\right)^{3} \mathrm{He}\left(\pi^{\circ}, \pi^{+}\right)^{3} \mathrm{H}-$ a very good description of the $\left(\gamma, \pi^{+}\right)$data over a wide range of photon energies and nuclear momentum transfers.

Finally, we have compared the process ${ }^{3} \mathrm{He}\left(\pi^{-}, \eta\right)^{3} \mathrm{H}$ with the few experimental data 


\section{PROGRESS}

and have made predictions for the as yet unmeasured $\eta$ photoproduction reactions on the trinucleon. For the former process we find that below $490 \mathrm{MeV}$ - the region below the free $\eta$ production threshold - our results for forward $\eta$ production significantly underestimate the data. We found good agreement at small momentum transfer and $T_{\pi}>560 \mathrm{MeV}$, but large deviations in the backward direction at large $\mathrm{Q}$. We believe that the same mechanism in all the incoherent processes is responsible for this puzzle that presents a clear indication of a breakdown of the impulse approximation.

${ }^{1}$ S.S. Kamalov, L. Tiator, and C. Bennhold, Phys. Rev. C447,941(1993).

${ }^{2}$ C. Bennhold et al., Nucl. Phys. A $\underline{540}, 621(1992)$.

${ }^{3} \mathrm{M}$. Espy et al., Proc. of the Intern. Conf. on Me'sons and Nuclei, Dubna, Russia, May 3-8, 1994 (in press).

${ }^{4}$ S. S. Kamalov, L. Tiator. and C. Bennhold, Few Body Systems 10,143(1991). 
PROGRESS

TOPIC: Cluster-Dynamical Approach to Nonrelativistic $N$-Body Scattering

INVESTIGATOR: H. Haberzettl

OBJECTIVE: To reformulate the nonrelativistic $N$-body scattering problem in terms of clusters instead of individual particles in order to provide a better starting point for a relativistic generalization which does not conserve the particle number.

SIGNIFICANCE: The nonrelativistic $N$-body problem is very well understood. ${ }^{1}$ It generalization to the relativistic many-body problem, however, is still in its infancy at present. One of the main reasons why the nonrelativistic problem in some sense is easier is the fact that the particle number is conserved in the nonrelativistic domain. In other words, it is possible, at least in principle, to trace each individual particle's evolution from the initial to the final state, through all possible intermediate reaction mechanisms. This is not the case for a truly relativistic scattering problem where the number of particles is not conserved. Another way of putting it is that a relativistic problem with full production and absorption degrees of freedom is always an infiniteparticle problem; as a consequence, the traditional $N$-body approach is bound to fail.

In view of this situation, it is interesting to see whether it is possible to reformulate the nonrelativistic scattering problem in such a way that it is blind to the fact that one deals with a fixed number of particles. A successful reformulation along these lines may then provide important clues as to how to approach the true relativistic problem.

DESCRIPTION OF PROGRESS: Instead of in terms of individual particles, a complete, consistent formulation of the nonrelativistic $N$-body scattering problem in terms of "clusters" was derived. ${ }^{2}$ A cluster is a subset of particles grouped together in a well-defined partition, with well-defined total momentum, total energy, total spin, etc. Within the cluster (which may or may not be able to form a bound state) all possible interactions are allowed. The cluster degrees of freedom, thus, comprise all possible (off-shell) scattering reactions among its constituents. Since it is well-known ${ }^{1}$ that it is possible to formulate the scattering problem entirely in terms of asymptotic two-cluster states (i.e., two initial and two final clusters), the relevant Hilbert space 36 of asymptotic states can be decomposed in terms two-cluster sectors $\mathfrak{H}_{\tau}=\mathfrak{H}_{\beta} \otimes \mathfrak{H}_{\alpha}$. The only irreducible transitions between asymptotic initial and final two-cluster sectors are then seen to be single-cluster exchange terms, with a diagrammatic structure similar to the time-ordered pieces of single-particle exchange Feynman diagrams. These "interactions", or driving terms, are described by vertices and propagators obtained from complete off-shell solutions of subsystem $T$ matrices via separable expansions. This $S$-matrix-type concept is realized by setting up the $N$-particle problem as the topmost step in a hierarchy of $n$-body scattering problems with increasing particle numbers $n=2,3, \ldots, N$ where the required input for the $n$-body subsystem problem is obtained from the complete solutions of all lower-level $k$-body problems (with $k<n$ ).

This hierarchical structure is well-known already from traditional Faddeev-type formulations of the $N$-body problem. ${ }^{1}$ Within the cluster approach it provides a natural ordering of scattering problems, which is seen to survive even in the relativistic generalization via the hierarchy of scattering problems distinguished by their conserved 
baryon number (see Refs. 3,4, and p. 34 in this report).

One of the most important features of the cluster approach, and of decisive importance for a relativistic generalization, is the fact that this formulation as a matter of course yields effective two-cluster integral equations in four variables, the usual threemomentum and an off-shell energy parameter. The latter comes about because in the cluster picture one must describe the intermediate propagation of two noninteracting subsystems sharing a given (fixed) energy, where one must integrate over all possible ways of sharing this energy. This is precisely what happens in a relativistic context when one integrates over the energy component of a four-momentum.

The cluster-dynamical formulation of the nonrelativistic $N$-body problem ${ }^{2}$ thus indeed provides important clues as to how to derive a relativistic generalization ${ }^{3,4}$. In fact, apart from the (nontrivial) kinematic aspects of such a generalization, the general structure of the ensuing relativistic formulation turns out to be exactly analogous to the present nonrelativistic results.

${ }^{1}$ S.K. Adhikari and K.L. Kowalski, "Dynamical Collision Theory and Its Applications" (Academic Press, Boston, 1991).

${ }^{2} \mathrm{H}$. Haberzettl, "Cluster-dynamical approach to $N$-body scattering", Phys. Rev. C466,687(1992).

${ }^{3} \mathrm{H}$. Haberzettl, "Relativistic cluster dynamics of nucleons and mesons. I: Kinematics and covariance", Phys. Rev. C47,1237(1993).

${ }^{4} \mathrm{H}$. Haberzettl, "Relativistic cluster dynamics of nucleons and mesons. II: Formalism and examples", Phys. Rev. C499,2142(1994). 
PROGRESS

TOPIC: Covariant Relativistic Cluster Dynamics of Nucleons and Mesons

INVESTIGATOR: H. Haberzettl

OBJECTIVE: To formulate a complete, consistent covariant description of relativistic scattering processes involving nucleons and mesons, including absorption and production of particles.

SIGNIFICANCE: Apart from the non-covariant approach of old-fashioned time-ordered perturbation theory, with its rather limited applicability, to date there exists no consistent dynamical formulation of the relativistic many-body scattering problem. It is evident, therefore, that such a formulation is of considerable significance from both theoretical and practical points of view.

DESCRIPTION OF PROGRESS: A time-ordered manifestly covariant relativistic scattering theory for arbitrarily large systems of nucleons and mesons was derived. ${ }^{1,2}$ The $S$-matrix-type approach is based on clusters rather than individual particles. It provides a recursive hierarchy of nonlinear scattering integral equations, each describing the dynamical evolution of two-cluster configurations at different levels of the manybody problem. The resulting effective two-body equations describe all possible absorption and production processes up to the maximum number of initially considered particles; they employ fully dressed cluster propagators and vertices, including all crossed meson contributions. Nonlinear couplings introduce effective contributions from infinitely many mesons.

Reference 1 provides a clarification of the kinematic aspects of the problem. It is shown there that the on-shell results of the formalism are Lorentz-covariant, without requiring anti-particle contributions. This was achieved by constructing off-shell $T$ matrices as invariants under an off-shell modification $\mathcal{H}$ of the Lorentz transformations $\mathcal{L}$. The transformation $\left(e^{\prime}, \overrightarrow{\mathbf{q}}^{\prime}\right)=\mathcal{M}(e, \overrightarrow{\mathbf{q}})$ of an arbitrary four-vector $(e, \overrightarrow{\mathbf{q}})$ describing a cluster with three-momentum $\vec{q}$ and off-shell energy $e$ is defined by relating the three-momenta $\overrightarrow{\mathbf{q}}^{\prime}$ and $\overrightarrow{\mathbf{q}}$ in two frames by Lorentz transformations involving only the respective on-shell energies $\omega^{\prime}$ and $\omega$, i.e., $\left(\omega^{\prime}, \overrightarrow{\mathbf{q}}^{\prime}\right)=\mathcal{L}(\omega, \overrightarrow{\mathbf{q}})$, where $\omega=\omega(m, \overrightarrow{\mathbf{q}})=\left(m^{2}+\overrightarrow{\mathrm{q}}^{2}\right)^{1 / 2}$, etc., with $m$ being the mass. The relation between off-shell energies $e^{\prime}$ and $e$ is then defined by

$$
e^{\prime}-\omega\left(m, \overrightarrow{\mathrm{q}}^{\prime}\right)=e-\omega(m, \overrightarrow{\mathrm{q}}),
$$

similar to Galilei transformations in Euclidean space. The modified transformations $\mathcal{M}$, therefore, leave the difference between on- and off-shell energies invariant, and reduce to $\mathcal{L}$ when going on-shell. It was realized in Ref. 2 that while $\mathcal{A}$ appears as a nonlinear transformation of four-vectors $(e, \vec{q})$, it can be understood as a covariant fourdimensional projection of a linear five-dimensional extension of the Lorentz transformations \&, i.e., Minkowski space is a hypersurface of an underlying fivedimensional manifold whose extra, fifth, dimension is interpreted as an off-shell energy component with transformation properties defined in (1), while the usual Minkowskispace energy component is mapped covariantly onto the mass shell. In essence, this new transformation may be thought of as interpolating between Galilei and Lorentz transformations, where full Lorentz-covariance is implemented only for on-shell matrix elements, with off-shell degrees of freedom being treated in a Galilei-like way. 
In view of the properties of the new transformations given above, as far as its spin is concerned a cluster behaves like an on-shell elementary particle of the same mass and. four-momentum. This allows one to treat the spin of a cluster explicitly in terms of known spinors, with known transformation properties; this is of considerable advantage in practical applications.

The details of the full implementation can be found in Ref. 2. It is shown that when including particle absorption and creation, the present approach leads to a recursive hierarchy of nonlinear scattering problems, where each hierarchical level is determined by the (conserved) number of baryons with the nonlinearity entailing contributions from infinitely many virtual mesons. The basic problem to be solved is pion-nucleon scattering; it is at this lowest level of the hierarchy that contact with field-theoretical approaches is made and that one needs to renormalize vertices in the usual way. At higher levels, renormalization will not be necessary. Furthermore, it is shown in Ref. 2 that the full exploitation of the nonlinearity inherent in the formalism leads to a fully crossing-symmetric solution of the pion-nucleon problem, which in turn may be used to obtain an equally fully crossing-symmetric formulation for the pion-deuteron problem.

Apart from the treatment of the spin, this relativistic formulation is formally equivalent to the non-relativistic $N$-body approach of Ref. 3 (see also p. 32 in this report). This equivalence may be of considerable importance from a practical point of view since it easily allows for a mixed treatment of situations in which some constituents must be treated relativistically while others may be incorporated nonrelativistically. (As an immediate application of such an approach, we discuss on pp. 36-42 in this report the relevant three-body forces for a three-nucleon system.)

The essential aspects of this formulation are understood and completed.1,2 There remain, however, a number of very interesting and potentially far-reaching theoretical and mathematical questions which will be continued to be investigated.

${ }^{1} \mathrm{H}$. Haberzettl, "Relativistic cluster dynamics of nucleons and mesons. I: Kinematics and covariance", Phys. Rev. C47,1237(1993).

${ }^{2} \mathrm{H}$. Haberzettl, "Relativistic cluster dynamics of nucleons and mesons. II: Formalism

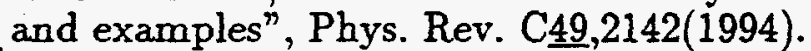

${ }^{3} \mathrm{H}$. Haberzettl, "Cluster-dynamical approach to $\mathrm{N}$-body scattering", Phys. Rev. C느,687(1992). 
PROGRESS

TOPIC: Triton Binding-Energy Calculation with a One-Pion-Exchange Three-Body Force

INVESTIGATORS: H. Haberzettl and W.C. Parke

OBJECTIVE: To solve the three-nucleon bound-state problem in a five-channel approximation with a novel type of three-body force to obtain an estimate of the size of such a contribution.

SIGNIFICANCE: Triton binding energies calculated with realistic nucleon-nucleon $(N N)$ interactions typically fall short of the experimental value of $8.48 \mathrm{MeV}$ by about 0.5-1 MeV. Attempts to resolve this discrepancy in terms of meson-exchange-based three-body forces provide encouraging but, at present, not entirely satisfactory results. The dynamically most detailed description of meson contributions in the three-nucleon system is provided by the coupled-channel approach pioneered by the Hannover group. Using the Paris potential, they find that employing explicit $\Delta$ channels raises the binding energy by $0.4 \mathrm{MeV}$ to $7.85 \mathrm{MeV}$, which still is about $0.6 \mathrm{MeV}$ short of the experimental value. The present novel type of three-body force has never been employed in any bound-state calculation.

DESCRIPTION OF PROGRESS: The cluster-dynamical treatment of meson-nucleon systems described in Ref. 1 also provides a consistent description of three-body forces in a three-nucleon system. It is found that there exists a one-pion-exchange three-body force which. to our knowledge, has never been taken into account in any calculation. In the usual coupled-channel approaches, such contributions either cannot be generated at all. or are discarded based on erroneous double-counting arguments. (A detailed analysis of these questions is given in Refs. 2,3 ; see also pp. 38-40 in this report.)

In order to get an estimate of the importance of this mechanism, we performed a fivechannel triton binding-energy calculation using only the new three-body force in addition to the purely nucleonic contributions. The latter were described by the Paris potential in a separable PEST expansion. We find that the purely nucleonic value of the binding energy is increased by about $0.6 \mathrm{MeV}^{4}$ As mentioned above, this value closely corresponds to the amount by which the binding energy obtained in coupledchannel calculations with explicit $\Delta$ degrees of freedom differs from experiment. We do not claim that the present result is the definitive solution of the triton puzzle. For a complete answer, one should perform a comprehensive calculation within the clusterdynamical framework outlined in Ref. 1. The binding-energy increase reported here may then be altered by the presence of other, competing mechanisms. However, the present findings show that the one-pion-exchange three-body force is an important mechanism which must be taken into account in a realistic calculation.

The results of this investigation are reported in Ref. 4; the underlying dynamical mechanisms are discussed Refs. 2,3 (see also pp. 38-40 in this report). A complete, consistent numerical investigation within the cluster approach is underway ( $\mathrm{Ph} . \mathrm{D}$. thesis project of A. Rakei; see pp. 41-42 in this report).

${ }^{1} \mathrm{H}$. Haberzettl, "Relativistic cluster dynamics of nucleons and mesons. II: Formalism and examples", Phys. Rev. C쓰,2142(1994). 


\section{PROGRESS}

${ }^{2} \mathrm{H}$. Haberzettl and W.C. Parke, "Relativistic origin of three-nucleon forces", in Contributed Papers, 14th International IUPAP Conference of Few-Body Problems in Phrsics (Williamsburg, VA, 26-31 May 1994), edited by Franz Gross (College of Ifilliam \& Mary/CEBAF, 1994), p. 881; and to be published in Proceedings of the 14th International IUPAP Conference of Few-Body Problems in Phvsics (Tilliamsburg, VA, 26-31 May 1994).

${ }^{3}$ H. Haberzettl and W.C. Parke, "Three-nucleon forces, relativity, and cluster dynamics three-nucleon forces", submitted for publication.

${ }^{4}$ H. Haberzettl and W.C. Parke, "Cluster-dynamical treatment of three-nucleon forces", in Proceedings of the XIVth European Conference of Few-Body Problems in Physics (Amsterdam, The Netherlands, 23-27 August 1993), edited by B.L.G. Bakker and R. van Dantzig, Few-Body Systems Suppl. 7,274(1994). 
PROGRESS

TOPIC: Three-Nucleon Forces, Relativity, and Cluster Dynamics

INIESTIGATORS: H. Haberzettl and W.C. Parke

OBJECTIVE: To provide a consistent description of the three-nucleon bound-state problem, including three-nucleon forces and relativistic meson-exchange effects.

SIGNIFICANCE: Up to now, despite decades of efforts, the theoretical value of the triton binding energy still remains one of the most elusive quantities with values calculated with realistic nucleon-nucleon $(N N)$ interactions typically falling short of the experimental value of $8.48 \mathrm{MeV}$ by about 0.5-1 MeV. Attempts to resolve this discrepancy in terms of meson-exchange-based three-body forces provide encouraging but. at present, not entirely satisfactory results. It is not entirely clear whether the origin of this shortcoming is to be found in an inadequate modeling of the reaction dynamics, in relativistic effects, or perhaps in both. In this situation, it is important to reexamine the three-nucleon bound-state problem from a point of view which allows for a consistent comparison of nucleons-only results, contributions from intermediate $\Delta$ degrees of freedom, and relativistic meson-exchange effects.

DESCRIPTION OF PROGRESS: The cluster-dynamical treatment of meson-nucleon systems described in Ref. 1 provides a particularly well-suited framework for investigating the effects of non-nucleonic contributions to the three-nucleon bound-state problem. Two aspects, in particular, are of importance here. First, the cluster approach is a manifestly covariant formalism with a straightforward nonrelativistic limit vielding precisely the usual Faddeev-type three-nucleon equations employed in nucleonsonly calculations. ${ }^{2-4}$ In view of the graphical structure of cluster dynamics ${ }^{1}$, the identification of relativistic effects can be done at a topological level and thus does not depend on arguments resting on, e.g., power-series expansions in $v / c$, which are known to be somewhat ambiguous. Second, since it is the very essence of that approach to describe subsystem reaction mechanisms in terms of the respective free off-shell $T$ matrices (and not in terms of potentials), by construction the double-counting of subsystem reaction mechanisms is not possible since such $T$ matrices are considered given, and their internal contributions do not appear at the explicit level.

In the simplest possible scenario going beyond the nucleons-only formulation, the threenucleon problem is described in terms of three single nucleons and one additional pion. The nucleons can interact via pairwise $N N$ forces, giving rise to an off-shell nucleonnucleon $T$ matrix, and the pion can be absorbed by a nucleon which either remains a nucleon or changes into a $\Delta$. The relevant nucleons-only asymptotic two-cluster sector of the Hilbert space comprises an interacting two-nucleon system and a single spectator nucleon. When projected onto this nucleonic $(2+1)$ structure of the asymptotic Hilbert space, the driving term $V$ for the three-nucleon bound-state problem is seen to contain four distinct contributions,

$$
V=V_{N}+V_{\Delta}+V_{\pi}+V_{\mathrm{NL}},
$$

where the nucleons-only term, $V_{N}$, describes the single-nucleon exchange transition between the initial and final $(2+1)$ Hilbert-space sectors. $V_{\Delta}$ comprises intermediate 
explicit $\Delta$ contributions very similar to what is obtained in the coupled-channel description of the Hannover model ${ }^{5}$. The remaining two terms, $V_{\pi}$ and $V_{\mathrm{NL}}$, comprise contributions which, to our knowledge, have never been taken into account in threenucleon calculations. The last term, $V_{\mathrm{NL}}$, contains a nonlinear contribution where a full three-nucleon $T$ matrix for transitions $(2+1) \rightarrow(2+1)$ is dressed by a pion and is presumably very small. The other term, $V_{\pi}$, describes the two time-ordered exchanges of a pion between the two-nucleon subsystem and the spectator nucleon. This term was already shown to contribute about $0.6 \mathrm{MeV}$ in a five-channel test calculation (see Ref. 4. and pp. $36-37$ in this report) and therefore definitely is not small.

This contribution has been criticized as double-counting pion-exchange contributions which would cancel against relativistic corrections of the nucleons-only term according to the investigations of Yang and Glöckle $(Y G)$ and Coon and Friar $(C F)^{6}$. Our in restigations show that such criticism is without foundation. In the lowest order of pion exchanges, $V_{\pi}$ is seen to comprise three pion exchanges, an initial and a final pion exchange between the same two nucleons, say between nucleons 1 and 2 , and one intermediate exchange between one of these two nucleons, say 2 , and the third nucleon 3 . The sequence of pion exchanges thus is $P_{12} P_{23} P_{12}$, where the indices label the nucleons between which exchanges take place. At least two of these exchanges are time-ordered such that they overlap, e.g., $P_{12}\left[P_{23} P_{12}\right]$, where the brackets indicate the orerlap. YG and $\mathrm{CF}$ showed that such overlaps $\left[P_{23} P_{12}\right]$ cancel exactly against relativistic corrections of the corresponding iterates $V_{23} V_{12}$ of the nucleon-nucleon potentials $V_{i j}$ which in view of the topological structure of the corresponding diagrams is an obviously correct result since the underlying Feynman diagrams do not contain loop integrations. In diagrams containing loop integrations, however, this cancellation is no longer true. In other words, the nonrelativistic limit of an entire relativistic loop diagram is not necessarily the same as a loop diagram constructed from nonrelativistic pieces, and this is precisely what happens here. In this respect, since it does not contain any non-nucleonic baryon contributions, the one-pion exchange three-nucleon potential $V$ may be called a true relativistic contribution. Its effect is that of an enhancement of the dominant one-pion exchange two-nucleon force in the medium of three nucleons.

Our investigations also show that the cluster picture does not give rise to the so-called dispersive contributions of the Hannover model $^{5}$. Nevertheless, similar effects, conceivably providing similar numerical contributions, are seen to emerge from $\Delta$ propagation in the presence of a fully interacting two-nucleon system. Again, such contributions follow from the nonrelativistic reductions of relativistic loop diagrams. They do not come from the explicit introduction of (energy-dependent) $\Delta$ loops at the three-nucleon level, which normally are subsumed already in the nucleon-nucleon $T$ matrix.

The present findings are detailed in Refs. 2,3. A complete, consistent numerical investigation within the cluster approach is underway ( $\mathrm{Ph} . \mathrm{D}$. thesis project of $\mathrm{A}$. Rakei; see pp. $41-42$ in this report).

${ }^{1} \mathrm{H}$. Haberzettl, "Relativistic cluster dynamics of nucleons and mesons. II: Formalism and examples", Phys. Rev. C499,2142(1994).

${ }^{2} \mathrm{H}$. Haberzettl and W.C. Parke, "Relativistic origin of three-nucleon forces", in Contributed Papers, 14th International IUPAP Conference of Few-Body Problems in 


\section{PROGRESS}

Physics (Williamsburg, VA, 26-31 May 1994), edited by Franz Gross (College of William \& Mary/CEBAF, 1994), p. 881; and to be published in Proceedings of the 14th International IUPAP Conference of Few-Bodv Problems in Physics (Williamsburg, VA, 26-31 May 1994).

${ }^{3} \mathrm{H}$. Haberzettl and W.C. Parke, "Three-nucleon forces, relativity, and cluster dynamics three-nucleon forces", submitted for publication.

${ }^{4} \mathrm{H}$. Haberzettl and W.C. Parke, "Cluster-dynamical treatment of three-nucleon forces", in Proceedings of the XIVth European Conference of Few-Body Problems in Physics (Amsterdam, The Netherlands, 23-27 August 1993), edited by B.L.G. Bakker and R. ran Dantzig, Few-Body Systems Suppl. 7,274(1994).

${ }^{5}$ Ch. Hajduk, P.U. Sauer, and W. Strueve, Nucl. Phys. A405,581(1983); Ch. Hajduk, P.U. Sauer, and S.N. Yang, ibid. A405,605(1983); M.T. Peña, H. Henning, and P.U. Sauer, Phys. Rev. C42,855(1990).

${ }^{6}$ S.N. Yang and W. Glöckle, Phys. Rev. C $\underline{33}, 1774(1986)$; S.A. Coon and J.L. Friar, Phys. Rev. C $\underline{34}, 1060(1986)$. 
TOPIC: Triton Binding-Energy Calculation with Three-Body Forces and Relativistic Effects

INVESTIGATORS: A. Rakei (Ph.D. Student), H. Haberzettl (Director), and W.C. Parke (Co-Director)

OBJECTIVE: To solve the three-nucleon bound-state problem with the three-body forces found in the cluster-dynamical treatment. ${ }^{1-3}$

SIGNIFICANCE: Triton binding energies calculated with realistic nucleon-nucleon $(N N)$ interactions typically fall short of the experimental value of $8.48 \mathrm{MeV}$ by about 0.5-1 MeV. Attempts to resolve this discrepancy in terms of meson-exchange-based three-body forces provide encouraging but, at present, not entirely satisfactory results. The dynamically most detailed description of meson contributions in the three-nucleon system is provided by the coupled-channel approach pioneered by the Hannover group. ${ }^{4}$ Using the Paris potential, they find that employing explicit $\Delta$ channels raises the binding energy by $0.4 \mathrm{MeV}$ to $7.85 \mathrm{MeV}$, which still is about $0.6 \mathrm{MeV}$ short of the experimental value. The results ${ }^{5}$ found for the one-pion exchange three-body force discussed on pp. 36-40 in this report suggest that the cluster-dynamical treatment may provide a more consistent framework for the calculation of three-body force effects than the usual coupled-channel approaches.

DESCRIPTION OF PROGRESS: The detailed outline of the project is given in Ref. 3 (see also pp. 36-40 in this report). The underlying nucleon-nucleon force to be taken is the Paris potential in a separable PEST expansion ${ }^{6}$. The code for the solution of the nucleons-only problem exists ${ }^{7}$ and will be amended for the inclusion of the three-body forces. The numerical code for describing the $\Delta$ as a dressed resonance according to the prescription of Ref. 1 is essentially completed. At present, the partial-wave analysis of the various contributing diagrams is being undertaken.

This work will be continued.

${ }^{1} \mathrm{H}$. Haberzettl, "Relativistic cluster dynamics of nucleons and mesons. II: Formalism and examples", Phys. Rev. C쎠,2142(1994).

${ }^{2} \mathrm{H}$. Haberzettl and W.C. Parke, "Relativistic origin of three-nucleon forces", in Contributed Papers, 14th International IUPAP Conference of Few-Body Problems in Physics (Williamsburg, VA, 26-31 May 1994), edited by Franz Gross (College of William \& Mary/CEBAF, 1994), p. 881; and to be published in Proceedings of the 14th International IUPAP Conference of Few-Body Problems in Physics (Williamsburg, VA, 26-31 May 1994).

${ }^{3} \mathrm{H}$. Haberzettl and W.C. Parke, "Three-nucleon forces, relativity, and cluster dynamics three-nucleon forces", submitted for publication:

${ }^{4} \mathrm{Ch}$. Hajduk, P.U. Sauer, and W. Strueve, Nucl. Phys. A405, 581 (1983); Ch. Hajduk, P.U. Sauer, and S.N. Yang, ibid. $\underline{\mathrm{A} 405}, 605$ (1983); M.T. Peña, H. Henning, and P.U. Sauer, Phys. Rev. C42, 855 (1990).

${ }^{5} \mathrm{H}$. Haberzettl and W.C. Parke, "Cluster-dynamical treatment of three-nucleon forces", in Proceedings of the XIVth European Conference of Few-Body Problems in Physics (Amsterdam, The Netherlands, 23-27 August 1993), edited by B.L.G. Bakker and R. van Dantzig [Few-Body Systems Suppl. I, 1994], p. 274. 


\section{PROGRESS}

${ }^{6} \mathrm{~J}$. Haidenbauer and W. Plessas, Phys. Rev. C30, 1822 (1984); ibid. 32, 1424 (1985); J. Haidenbauer, Y. Koike, and W. Plessas, ibid. $\underline{33}, 439$ (1986); J. Haidenbauer and Y. Koike, ibid. $\underline{34}, 1187$ (1986).

'W.C. Parke, Y. Koike, D.R. Lehman, and L.C. Maximon, Few-Body Systems 11.89(1991). 
PROGRESS

TOPIC: Calculation of Pion-Nucleon Scattering within the Relativistic ClusterDynamical Approach

\author{
I.ITESTIGATOR: H. Haberzettl
}

OBJECTIVE: To solve the pion-nucleon problem within the cluster-dynamical framework of the relativistic $N$-body problem and to represent the resulting pion-nucleon amplitudes such that they may serve as input in other problems with explicit mesonic degrees of freedom. One result is to be a self-consistently calculated off-shell $\pi N-N$ form factor.

SIGNIFICANCE: As is described on pp. 34-35 in this progress report, pion-nucleon scattering is the basic problem to be solved for all applications of the relativistic clusterdrnamical (RCD) approach ${ }^{1}$; it is at the same level of importance as the two-nucleon problem for the nonrelativistic $N$-body problem. Without its solution, applications of the RCD approach to higher-order processes, like nucleon-nucleon and pion-deuteron scattering, cannot be done consistently.

DESCRIPTION OF PROGRESS: The nonlinearity inherent in the RCD approach ${ }^{1}$ is one of its most essential aspects. At present, we are engaged in a numerical study of these nonlinearities for several simplified versions of the pion-nucleon problem. One important result for these simple models is that the convergence of the iterated nonlinearities depends crucially on which of several possible functions one chooses to iterate. The finding that only dimensionless combinations of functions can be expected to have sufficiently benign convergence properties to be of practical usefulness can presumably be expected also to be true for more complicated realistic cases. The simple models will successively be made more complex and realistic. This careful approach is warranted since there exists very little experience for the solution of nonlinear integral equations.

The partial-wave analysis of all contributing diagrams is almost completed. The effect and magnitude of single-loop diagrams is being studied for some simplified cases.

This work will be continued.

${ }^{1} \mathrm{H}$. Haberzettl, "Relativistic cluster dynamics of nucleons and mesons. II: Formalism

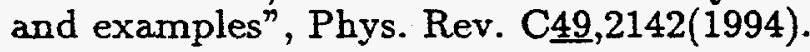


PROGRESS

TOPIC: Covariant Model for Dynamical Quark Confinement

INTESTIGATOR: H. Haberzettl

OBJECTIVE: To derive a model for the confinement of quarks based on quark propagators which do not admit singularities for real energies.

SIGNIFICANCE: Although quantum chromodynamics (QCD) is generally held to be the fundamental theory of the strong interaction, to date there exists no direct derivation of the dynamical mechanism of quark confinement. A consistent description of confined quarks in a dynamical model such that it may be utilized for the calculations of mesonic or baryonic scattering processes is desirable and may be of significant help for modeling and understanding the dynamical mechanisms of mesonbaryon reactions and, in particular, of electromagnetic processes to be measured at, e.g., CEBAF.

DESCRIPTION OF PROGRESS: A model for the quark propagator is considered where one describes the dressed propagator via a gluon-loop expansion with bare quarkgluon vertices with a massless bare quark. The quark inside the gluon loop is taken to be dressed. In other words, one has an integral equation for the quark propagator $t$ given by

$$
t=t_{b}+t_{b} \Sigma[t] t
$$

where $t_{b}$ is the bare propagator and the self-energy loop $\Sigma[t]$ is a functional of $t$. The structure of this equation is similar to a nonlinear Dyson-Schwinger equation in the socalled rainbow approximation. ${ }^{1}$ The difference is that we consider this equation in terms of time-ordered propagating quarks and gluons according to the manifestly corariant time-ordered cluster-dynamical scattering formulation of Ref. 2. As already discussed pp. 34-35 in this report, this approach provides a direct formulation of offshell degrees of freedom in terms of an energy variable having Euclidean properties without requiring one to work in an explicit. Euclidean metric (which usually is the preferred metric for QCD investigations ${ }^{1}$ ).

Assuming the usual Dirac structure for the dressed quark propagator $t$, one finds that the dynamical (i.e., energy-dependent) quark mass $m$ is determined by a highly nonlinear homogeneous integral equation which symbolically can be written as

$$
m=\Sigma[m]
$$

where $\Sigma[m]$ is $\Sigma[t]$ but now taken as a functional of $m$. Without any calculation, simply from invariance requirements, one finds that $m$, in the rest frame of the quark, has the form

$$
m(E)=\frac{E}{2}+\sqrt{\left(\frac{E}{2}\right)^{2}+m_{0}^{2}(E)}
$$

where $m_{0}^{2}(E)$ is an unknown function. The function $m(E)$ is such that it vanishes for 
PROGRESS

large negative energies and rises to infinity monotonically for $E$ going to $+\infty$. Since $m(E)>E$ for all finite $E$, this clearly shows that such an $m(E)$ is confining, i.e., the corresponding propagator does not have a pole for real energies (which would require that $m(E)$ possesses a fixed point $E_{0}=m\left(E_{0}\right)$ ).

Treating the gluon in the self-energy integral $\Sigma[m]$ of $(1)$ like a bare photon, one finds two contributions, one arising from transverse and one from longitudinal and scalar gluons. For the latter contribution, the self-energy integral is seen to converge, without any need for regularization. The transverse piece, however, diverges except in the softgluon limit. We have chosen, therefore, to neglect this piece and have taken into account only longitudinal and scalar gluon contributions; this corresponds to considering only purely Coulombic gluons. This treatment has the added advantage that this provides a simple mechanism of gluon confinement since longitudinal and scalar gluons are not observable. (Alternatively, one may renormalize the mass by subtracting the divergent piece from both sides of (1).)

A numerical solution of (1) then indeed yields a dynamical quark mass which for negative and low positive energies may be described by (2) with a constant $m_{0}$, with the value of that constant providing the mass scale. For all energies, the monotonically rising function $m(E)>E$; this $m(E)$ thus is indeed confining. In view of the fact that (1) may be viewed as a (nonlinear) eigenvalue equation, this nontrivial solution corresponds to a fixed quark-gluon coupling constant eigenvalue of $g^{2} / 4 \pi=4.712$. This value was determine numerically; in actual fact, by considering the soft-gluon limit of the integral equation, one can show that the exact result is

$$
\frac{g^{2}}{4 \pi}=\frac{3 \pi}{2}
$$

The value of this coupling constant is not unreasonable for the low-energy limit of quark-gluon coupling. It is a true constant; to obtain a running coupling constant, one would have to include dressing mechanisms for the vertex.

The dynamical mass $m(E)$ obtained in the present time-ordered description is not the same as the mass $m_{F}\left(E^{2}\right)$ of a Feynman-type formulation. The two are related by

$$
r\left(E^{2}\right)[E-m(E)][E+m(-E)]=E^{2}-m_{F}^{2}\left(E^{2}\right),
$$

where

$$
r\left(E^{2}\right)=\frac{2 m(-E) m(E)}{E[m(-E)-m(E)]+m^{2}(-E)+m^{2}(E)}
$$

This expression for $m_{F}$ is symmetric in $E$ and $-E$ and thus $m_{F}$ can be viewed as a function of $E^{2}$, as must be the case for a mass in a Feynman-type formulation.

This difference between $m$ and $m_{F}$ actually can be shown ${ }^{3}$ to be responsible for the fact that for a Lorentz-covariant formulation of dynamical confinement there exist no spinor solutions for the Dirac equation, which presents problems for the corresponding fieldtheoretic foundation. In the present time-ordered approach, by contrast, Dirac spinors still exist. This suggests that the latter may perhaps be better suited for such 
investigations.

A manuscript with these findings was submitted for publication. ${ }^{3}$ Further inrestigations concerning the dressed structure of the quark-gluon vertex and of the resulting running coupling constant are in progress.

${ }^{1}$ For a review of these and related questions, and further references, see C.D. Roberts and A.G. Williams, in, "Progress in Particle and Nuclear Physics", edited by A. Faessler (Pergamon Press, Oxford, 1994).

${ }^{2} \mathrm{H}$. Haberzettl, "Relativistic cluster dynamics of nucleons and mesons. II: Formalism ard examples", Phys. Rev. C499,2142(1994).

${ }^{3} \mathrm{H}$. Haberzettl, "Covariant off-shell model for dynamical quark confinement", submitted for publication. 
PROGRESS

TOPIC: Experimental and Theoretical Investigation of the ${ }^{2} H(n, n n p)$ Reaction and of the Veutron-Neutron Scattering Length

INVESTIGATORS: K. Gebhardt, W. Jäger, C. Jeitner, M. Vitz, E. Finckh (all Universität Erlangen, Germany), T.N. Frank, Th. Januschke, W. Sandhas (all Universität Bonn, Germany), and H. Haberzettl.

OBJECTIVE: To extract the neutron-neutron scattering length from the final-state interaction data of the kinematically complete Erlangen neutrondeuteron breakup experiment.

SIGNIFICANCE: Assuming charge symmetry, one would expect the scattering lengths for proton-proton (pp) and neutron-neutron (nn) scattering to be identical. Experimentally, however, the (Coulomb-corrected) value for the pp scattering length is found to be ${ }^{1}$

$$
a_{p p}=-17.3 \pm 0.3 \mathrm{fm},
$$

while the nn values scatter between ${ }^{2}$

and

$$
a_{n n}=-16.4 \pm 1.2 \mathrm{fm}
$$

$$
\mathrm{a}_{\mathrm{nn}}=-18.8 \pm 1.0 \mathrm{fm} .
$$

Clearly, the current situation with respect to charge-symmetry breaking is inconclusive. Therefore, a reliable determination of the nn scattering length is important - not only with respect to the question of charge-symmetry breaking, but also since $a_{\text {mn }}$ is a very crucial parameter for realistic nucleon-nucleon interactions used in few-body calculations. Its accurate value, therefore, is of significance for all theoretical investigations in few-nucleon systems.

DESCRIPTION OF PROGRESS: In order to determine the nn scattering length from nd $\rightarrow$ nnp breakup data, one needs to investigate the situation in which the two final neutrons leave the interaction volume with a vanishing relative momentum. This finalstate interaction (FSI) of the two neutrons is dominated by the two-body ${ }^{1} \mathrm{~S}_{0}$ partial wave. By performing a full three-body breakup Faddeev calculation and fitting the measured FSI peak with theoretical results due to various input values of $a_{n n}$ for the ${ }^{1} S_{0}$ subsystem channel, one obtains a best fit which thus determines the scattering length. In principle, this procedure is straightforward. In practice, however, it is a nontrivial task in view of the enormous numerical requirements.

In the Erlangen nd breakup experiment, one measures the kinematic variables of the two final neutrons. This is very difficult to do and requires great care in analyzing the experimental raw data. Ideally, the measured values should sit on a curve in the plane spanned by the two neutron energies which is defined as the kinematic locus of neutron energies satisfying three-body energy conservation. In practice, however, due to the finite resolutions of the neutron detectors, the experimental data scatter quite a bit around this ideal curve. In order to extract cross sections, which are usually given as functions of the arc length along the kinematic curve, one therefore needs to project the measured values onto appropriate positions on the kinematic locus. 
Previously, this projection was done by performing a Monte Carlo simulation of the experiment. It was found, however, that this procedure was inadequate to provide the desired accuracy. We therefore performed a numerical simulation based on complete solutions of the Faddeev-type AGS equations for the breakup problem; as input we used the separable $W$-matrix representation ${ }^{3}$ of the Paris potential. Since each cross section is characterized by the energy of the incident neutron, the scattering angles $\theta_{1}$ and $\theta_{2}$ of the two final neutrons and the difference $\Delta \phi$ of their azimuthal angles, an adequate description of the finite detector resolutions requires variations of all four kinematic variables. We found that we had to calculate up to 375 different kinematic configurations to analyze one experimental cross section, i.e., we used 3 different energies and five different angles for each of the three angle variables (i.e., $37 \overline{5}=3 \times 5 \times 5 \times 5)$. Details and results for the latter for rearrangement and breakup neutron-deuteron scattering calculations with the Paris potential are given in Ref. 4 .

In order to extract the nn scattering length from the data, we performed several sets of 375 calculations for an experimental situation dominated by the final-state interaction of the two neutrons, with each set employing different input values for the nn scattering length $a_{n n}$. Subjecting then the theoretical results to the same procedure usually used for the experimental data and performing a best fit for the FSI peak of the cross section, we find a value of

$$
a_{\mathrm{mn}}=-17.0 \pm 1.0 \mathrm{fm}
$$

for the nn scattering length, which is compatible with the pp scattering length.

Preliminary results of our analysis were reported at conferences at Adelaide and Salzburg ${ }^{5}$; our final results are published in Ref. 6.

${ }^{1}$ See R. Machleidt, Adv. Nucl. Phys. 19,189(1989), and references therein.

${ }^{2}$ G.F. Téramond and B. Gabioud, Phys. Rev. C $36,691(1987) ;$ N. Koori et al., Book of Contributions to the 11th International IUPAP Conference on Few Body Systems in Particle and Nuclear Physics (Tokyo and Sendai, Japan, August 24-30, 1986), edited by T. Sasakawa et al., Suppl. to Research Report of the Laboratory of Nuclear Science, Vol 19, p. 406 (Tohoku Univ., 1986); I. Slaus, Few Body Systems, Suppl. 1,160(1987); K. Bodek et al. Few Body Systems 8,23(1990), and references therein.

${ }^{3}$ E.A. Bartnik, H. Haberzettl, and W. Sandhas, Phys. Rev. C34,1520(1986); E.A. Bartnik, H. Haberzettl, Th. Januschke, U. Kerwath, and W. Sandhas, Phys. Rev. Cㅌ6,1678(1987); T.N. Frank, H. Haberzettl, Th. Januschke, U. Kerwath, and W. Sandhas, Phys. Rev. C38,1112(1988).

${ }^{4}$ Th. Januschke, T.N. Frank, W. Sandhas, and H. Haberzettl, "Neutron-deuteron scattering calculations with realistic $N N$ interactions using the W-matrix representation of the two-body input", Phys. Rev. C417,1401(1993); T.N. Frank, Ph.D. Thesis (Universität Bonn, 1992).

${ }^{5}$ K. Gebhardt, W. Jäger, E. Finckh, T.N. Frank, Th. Januschke, W. Sandhas, and H. Haberzettl, in Proceedings of the XIIIth International Conference on Few-Body Problems in Physics (Flinders University, Adelaide, Australia, 5-11 January 1992), edited by I.R. Afnan and R.T. Cahill, p. 152; in Proceedings of the Spring Meeting of the Nuclear Physics Section of the German Physical Society (Salzburg, Austria, 24-28 
February 1992), Verhandl. DPG (VI) 27, p. 121.

${ }^{6} \mathrm{~K}$. Gebhardt, W. Jäger, C. Jeitner, M. Vitz, E. Finckh, T.N. Frank, Th. Januschke, IV. Sandhas, and H. Haberzettl, "Experimental and theoretical investigation of the ${ }^{2} \mathrm{H}(\mathrm{n}, \mathrm{nnp})$ reaction and of the neutron-neutron scattering lengtb", Nucl. Phys. $1561.232(1993)$. 
PROGRESS

TOPIC: Neutron-Deuteron Scattering Calculations with Realistic $N N$ Interactions Using the $W$-Matrix Representation of the Two-Body Input

INTESTIGATORS: Th. Januschke, T.N. Frank, W. Sandhas (all Universität Bonn, Germany), and H. Haberzettl.

OBJECTIVE: To calculate observables for elastic neutron-deuteron scattering, and to demonstrate the usefulness of the separable $W$-matrix representation ${ }^{1}$ of the two-nucleon input for three-nucleon scattering calculations.

SIGNIFICANCE: Three-nucleon scattering calculations for realistic potentials are among the most demanding numerical tasks of few-body physics. In calculations for semirealistic potentials, it was shown that this computational efforts can be considerably reduced, with only minor sacrifices in accuracy, if one employs the separable $W$-matrix representation to simplify the required two-nucleon input. The present investigation shows that this is also true for realistic interactions like the Paris potential. This is of particular relevance for investigations in which one must calculate a large number of different configurations.

DESCRIPTION OF PROGRESS: Calculating a complete set of observables for elastic neutron-deuteron scattering for an optimized $W$-matrix representation of the Paris potential, it was shown ${ }^{2}$, by comparison with extant direct-integration results ${ }^{3}$, that the results of the simplified $W$-matrix approach in the majority of cases coincide with direct-integration results within the respective numerical errors. For a few observables, larger differences are found which, in all such cases, can be traced back to a not quite optimal $W$-matrix representation of the two-nucleon p-wave input.

A summary of our results is published in Ref. 2.

${ }^{1}$ E.A. Bartnik; H. Haberzettl, and W. Sandhas, Phys. Rev. C3441520(1986); E.A. Bartnik, H. Haberzettl, Th. Januschke, U. Kerwath, and W. Sandhas, Phys. Rev. C 36, 1678(1987); T.N. Frank, H. Haberzettl, Th. Januschke, U. Kerwath, and W. Sandhas, Phys. Rev. C38,1112(1988).

${ }^{2}$ Th. Januschke, T.N. Frank, W. Sandhas, and H. Haberzettl, "Neutron-deuteron scattering calculations with realistic $N N$ interactions using the $W$-matrix representation of the two-body input", Phys. Rev. C47,1401(1993).

${ }^{3} \mathrm{H}$. Witała, T. Cornelius, and W. Glöckle, Few-Body Systems $\underline{3}, 123(1988) ; \mathrm{H}$. Witała, W. Glöckle, and T. Cornelius, Nucl. Phys. A491,157(1989). 
PROGRESS

TOPIC: D-State Structure of ${ }^{4} \mathrm{He}$

INVESTIGATORS: H. Ito, H. Haberzettl, D.R. Lehman, and B. F. Gibson (LANL).

OBJECTIVE: To solve the four-nucleon bound-state equations by Faddeev methods with realistic two-nucleon interactions in order to investigate the $D$ state properties of ${ }^{4} \mathrm{He}$.

SIGNIFICANCE: Solution of the four-nucleon bound-state problem is intrinsically difficult. So far, it has been solved with the tensor force present by Faddeev methods only for the separable rank-1 interactions of Yamaguchi, ${ }^{1}$ whereas, with realistic twonucleon interactions, it has been calculated by variational methods, ${ }^{2}$ variational Monte Carlo techniques, ${ }^{3}$ Green's function Monte Carlo methods, ${ }^{4}$ and by the ATMS (Amalgamation of Two-body correlations into Multiple Scattering processes). ${ }^{5}$ Whereas the Faddeev solutions should be numerically reliable to investigate the D-state properties of the ${ }^{4} \mathrm{He}$ nucleus, e.g., the ${ }^{4} \mathrm{He} \rightarrow \mathrm{d}+\mathrm{d}$ asymptotic normalization constants, the underlying interactions have a significant deficit in their representation of the twonucleon tensor force. On the other hand, the solutions that have been obtained with realistic two-nucleon interactions tend not to be numerically well determined in the asymptotic region ${ }^{6}$ where one needs convergence to extract the $\mathrm{D}$-state parameters like the asymptotic normalization constants and the Distorted Wave Born Approximation parameter, $D_{2}$. Thus, wave functions obtained for the ${ }^{4} \mathrm{He}$ ground state by Faddeev methods with realistic two-nucleon interactions should permit us to predict reliable values for the asymptotic normalization constants and the DWBA parameter $\mathrm{D}_{2}$. As can be seen in the review by Lehman, ${ }^{7}$ the situation with regard to the D-state properties of ${ }^{4} \mathrm{He}$ is very unsettled both theoretically and experimentally, so the proposed theoretical work should help to clarify the situation. Moreover, the availability of such wave functions will permit us to calculate momentum distributions for ${ }^{4} \mathrm{He} \rightarrow \mathrm{n}+{ }^{3} \mathrm{He}, \rightarrow \mathrm{p}+{ }^{3} \mathrm{H}$, and $\rightarrow \mathrm{d}+\mathrm{d}$, all relevant to coincidence electron scattering experiments already carried out at MIT and NIKHEF, and planned for CEBAF.

DESCRIPTION OF PROGRESS: In solving four-body, bound-state problems, it is more transparent to begin with Schrodinger's equation and to solve the integral equations for the spectator functions defined below. This is our general direction ${ }^{8}$. It is quite different from the conventional $t$-matrix approach of the original Yakubovsky equation ${ }^{9}$ and subsequent extensions ${ }^{10,11}$, where an approximation of the Bateman or Hilbert-Schmidt expansion type is used in the kernel of the equations. Once the realistic NN interaction is expressed in the separable form, our integral equations for the spectator functions are exactly soluble by using today's super-computer facilities. Furthermore, this exact treatment of the kernel becomes essential when calculating the asymptotic normalization constants: since the asymptotic separation takes place below the threshold of the on-shell-subcluster system, the normalization constant is expressed in terms of the four-body bound state wave function at an imaginary momentum. This requires the analytical continuation of the wave function, which can be implemented exactly only by using the exact form of the kernel. This point is obscure in the frontier work ${ }^{12}$ of this topic. In addition, the use of separable potentials greatly simplifies this procedure: the Faddeev components of the $(3+1)$ and $(2+2)$ configurations, $\psi(12,3 ; 4)$ and $\psi(12 ; 34)$ respectively, can be given in the factorizable form composed of the spectator function $Q(p, q)$ or $R(t, s)$ and the form factor of the separable potential, $g(k)$, 
PROGRESS

multiplied by the four-body Green's function $\left(H_{0}+B\right)^{-1}$,

and

$$
\psi(12,3 ; 4)=g(k) Q(p, q) /\left(H_{0}+B\right)
$$

$$
\psi(12 ; 34)=g(k) R(t, s) /\left(H_{0}+B\right) .
$$

Here, the functional form is explicitly known for $g(k)$ and $\left(H_{0}+B\right)$, so that the analytical continuation for the variable $q$ in $Q(p, q)$ (also for $s$ in $R(t, s)$ can be simply implemented by using the integral equation. The accurate numerical procedure is quite feasible by computer as has been applied for the similar problem in three-body systems ${ }^{13}$. In contrast, we would have had to deal with a more involved form of the analytical continuation if we followed the direct Faddeev approach ${ }^{14}$ using the local form of $\mathrm{NN}$ interactions. We have divided the present program into several levels of derelopment:

[Level-1] Solve the 4-boson problem including a few partial waves $(l=0,1,2)$ with rank-one separable interaction to check partial-wave convergence.

[Level-2] Solve the 4-fermion problem with spin-dependent central forces for $1=0$ : (i) derive the integral equations with the spin degrees of freedom;

(ii) solve the equations in (i) with spin-dependent interactions $V\left({ }^{1} S_{0}\right)$ and $V\left({ }^{3} S_{1}\right)$

(iii) take $V\left({ }^{1} S_{0}\right)=V\left({ }^{3} S_{1}\right)$ limit in (i) and (ii) to check correctness of code (iv) repeat (i)-(iii) with $l=0,1,2$ to check convergence with partial waves.

[Level-3] Normalize the wave function and calculate the $S$-wave asymptotic normalization constants of the $(3+1)$ and $(2+2)$ separations. The former applies to the ${ }^{4} \mathrm{He} \rightarrow{ }^{3} \mathrm{He}+n$ process.

[Level-4] Solve the four-fermion problem including the tensor and central forces:

(i) derive the integral equations with these forces present;

(ii) solve the equations from (i) and obtain the normalized wave function;

(iii) obtain the D-state probability and the asymptotic S- and D-wave normalization constants.

[Level-5] Repeat Level-4 with Paris NN interaction through use of separableexpansion methods as given by Refs. 15-17, for example.

We have completed the work through level-3. In particular, we find a linear correlation between the $S$-wave asymptotic normalization of the ${ }^{4} \mathrm{He} \rightarrow{ }^{3} \mathrm{He}+n$ separation $\left(C_{l=0}\right)$ and the difference of the three- and four-body binding energies; $\quad C_{l=0} \propto B_{4}-B_{3}$, the same conclusion as found in the three-body problem ${ }^{13}$. A paper is in preparation that describes these results. We are now about to move to the central issue: investigation of the tensor force in ${ }^{4} \mathrm{He}$ and calculation of the $\mathrm{S}$ - and D-wave asymptotic normalization constants. We propose the completion of levels 4 and 5 within the next two years.

${ }^{1}$ A.C. Fonseca, Phys. Rev. C40,1390(1989); Nucl. Phys. A $\underline{508,281 c(1990) .}$

${ }^{2}$ J.L. Ballot, Phys. Lett. 127 $\bar{B}, 399(1983) ;$ P. Goldhammer, Phys. Rev. C29, 1444(1984);

P.C. Chiang, M.R. Meder, and J.E. Purcell, Phys. Lett. 235B,225(1990).

${ }^{3}$ R. Schiavilla, V.R. Pandharipande, and R.B. Wiringa, Nucl. Phys. A449,219(1986).

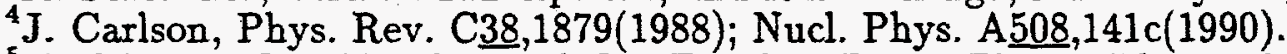

${ }^{5}$ H. Morita, Y. Akaishi, and H. Tanaka, Prog. Theor. Phys. 79,1279L(1988); J.A.

Tostevin, in Proc. Tsukuba Int. Workshop on Deuteron Involved Reactions and 
PROGRESS

Polarization Phenomena, Tsukuba, Japan (World Scientific, Singapore, 1985), p.241; J. Phys. G11,L211(1985).

${ }^{6} \mathrm{~J}$. Carlson and V.R. Pandharipande, private communication.

'D.R. Lehman, in Les Editions de Physique, Colloque de Physique, Colloque C6. Supplement 22, 51, C6-47(1990).

${ }^{8}$ B.F. Gibson and D.R. Lehman, Phys. Rev. C14,685(1976).

${ }^{9}$ O.A. Yakubovsky, Yad. Fiz. $\underline{5}, 1312(1967)$ [Sov. J. Nucl. Phys. $\left.\underline{5}, 937(1967)\right]$.

${ }^{10}$ J.A. Tjon, Phys. Lett. B $\underline{56}, 217(1975)$.

${ }^{11}$ A.C. Fonseca, Proceedings of te 8th Autumn school on Few-Body Physics at Lisboa, Portugal, edited by L.S. Ferreira, A.C. Fonseca, and L. Streit (Springer-Verlag, 1987) p. 161, and references therein.

${ }_{12}$ A.G. Bartshnikov, L.D. Blokhintsev, and I.M. Narodetsky, Nucl. Phys. A272,327(1976).

${ }^{13}$ B.F. Gibson and D.R. Lehman, Phys. Rev. C29,1017(1984).

${ }^{14} \mathrm{H}$. Kamada and W. Glöckle, Phys. Rev. Lett. 71,971(1993).

${ }^{15}$ W.C. Parke, Y. Koike, D.R. Lehman, and L.C. Maximon, Few-Body Systems $11,89(1991)$.

${ }^{16}$ D.J. Ernst, C. M Shakin, R.M. Thaler, Phys. Rev C8,46(1973); ibid, Cg, 1780(1974); J. Haidenbauer and W. Plessas, Phys. Rev. C $30,1822(1984)$; J. Haidenbauer and Y. Koike, Phys. Rev. C34,1187(1986).

${ }^{17}$ Y. Koike, Phys. Rev. C42,R2286(1990); private communication. 
PROGRESS

TOPIC: Gauge Invariance and Compton Scattering from Relativistic Few-Body Systems

INTESTIGATOR: Hiroshi Ito, Franz Gross (CEBAF and College of William \& Mary)

OBJECTIVE: To develop a theoretical basis for the gauge invariance of inelastic and/or inclusive electron scattering from relativistic composite systems and to calculate numerically the amplitude of Compton scattering with tractable models for few-body systems.

SIGNIFICANCE: Inclusive electron scattering from a relativistic composite system is of great interest in association with today's high-energy, high-efficiency experimental facilities such as the Continuous Electron Beam Accelerator Facility (CEBAF). Interesting scaling phenomena are observed in this process, for example, $x$-scaling for the parton model in hadron physics and $y$-scaling ${ }^{1,2}$ in nuclear physics. The response tensor of inclusive electron scattering can be expressed as an integral over the expectation value of the current commutator

$$
W^{\mu \nu}=\int d^{4} x e^{i q x}<g s\left|\left[J^{\mu}(x), J^{\nu}(0)\right]\right| g s>,
$$

where $|g s\rangle$ is the ground-state wave function of the composite system. The response tensor, $W^{\mu \nu}$, is related to the imaginary part of the virtual Compton amplitude: $W^{\mu \nu} \propto$ $I_{m} T^{\mu \nu}$, and this amplitude must satisfy the gauge invariance; $q_{\mu} T^{\mu \nu}=0$. This requirement can be satisfied only with a dynamically consistent treatment of (a) the bound-state wave function, (b) rescattering in the intermediate continuum state, and (c) the electromagnetic two-body current operators. This problem has been extensively investigated in the framework of nonrelativistic dynamics ${ }^{3}$, but very few ${ }^{4}$ of the relativistic studies have ever been explicitly developed. Within the Bethe-Salpeter formalism (or its reduced form in three-dimensions), we develop a dynamical theory of gauge invariant Compton scattering. An explicit solution to the problem will be obtained by using covariant separable interactions, and will shed light on the relativistic dynamics in the inclusive electron scattering from composite systems. The physics of $y$ scaling will be studied within a consistent framework.

DESCRIPTION OF PROGRESS: The theoretical formulation of Compton scattering has been completed for the relativistic two-body systems, and published ${ }^{5}$, with the following outcomes:

(1) In both electrodisintegration and Compton scattering processes, if nuclear forces are nonlocal the conventional amplitude including the Impulse(IMP) and Final State Interaction (or continuum rescattering) processes is not gauge invariant.

(2) The nonlocal two-body potential can be expressed as $V\left(x_{1}^{\prime}, x_{2}^{\prime} ; x_{1}, x_{2}\right)$ in the coordinate representation, where $x_{1}^{\prime}$ and $x_{2}^{\prime}\left(x_{1}\right.$ and $\left.x_{2}\right)$ are the coordinates of the 1 st and 2-nd particle at the final (initial) state. Within the nonlocal length defined by $d=\left|x_{1}^{\prime}-x_{1}\right| \sim\left|x_{2}^{\prime}-x_{2}\right|$, photons may interact with the charged constituents participating in the NN interaction, and this induces an additional current, namely a two-body interaction current. Including this contribution, the total amplitude becomes gauge invariant. 
(3) We have derived the explicit form of the interaction currents from four different models: (a) one-pion-exchange potential; (b) two-pion-exchange potential; (c) covariant separable potential ${ }^{6}$; and (d) $\rho \pi \gamma$ - and $\omega \sigma \gamma$-conversion processes ${ }^{7}$. These current operators satisfy the two-body Ward-Takahashi identity ${ }^{8,9}$.

The low-energy limit of the Compton amplitude, for example the Thomson-limit, should be derivable within any dynamical framework. The importance of the negative energy state should be clarified within the relativistic dynamics of Compton scattering. The electromagnetic polarizability of composite systems may depend sensitively on the rescattering in the intermediate continuum state. In order to investigate these issues, a numerical calculation is in progress for Compton scattering from a relativistic two-body system, where the explicit evaluation of the rescattering and interaction current processes is to be completed with the use of covariant separable interactions. As a proposed project for the next three-year period, we expect to extend the present framework to relativistic three-body systems, where the dynamics is described by the relativistic form of the Faddeev equations.

${ }^{1}$ G.B. West, Phys. Rep. 18C, 264(1975).

${ }^{2}$ W. M. Alberico, A. Molinari, T.W. Donnelly, E.L. Kronenberg, and J.W. van Orden, Phys. Rev. C 38, 1801(1988).

${ }^{3}$ J.L. Friar and S. Fallieros, Phys. Rev. C34,2029(1986); Phys. Rev. C42,2246(1990); J. L. Friar, Ann. Phys. (N.Y.) 95,170(1975); A.C. Hayes, J.L. Friar, and D.D. Strottman, Phys. Rev. C41,1727(1990).

${ }_{5}^{4}$ S.D. Drell and T.D. Lee, Phys. Rev. D5,1738(1972).

${ }^{5} \mathrm{H}$. Ito and F. Gross, Phys. Rev. C48, 1948(1993); CEBAF preprint, \#CEBAF-TH-9310.

${ }^{6} \mathrm{H}$. Ito, W.W. Buck, and F. Gross, Phys. Rev. C43,2483(1991).

${ }^{7} \mathrm{H}$. Ito and F. Gross, Phys. Rev. Lett. $\underline{11}, 2555(1993)$; CEBAF preprint, \#CEBAF-TH93-06.

${ }^{8}$ F. Gross and D.O. Riska, Phys. Rev. C $36,1928(1987)$.

${ }^{9}$ K. Ohta, Phys. Rev. C느, 1335(1989); C1ㅣ,1213(1990). 
PROGRESS

TOPIC: Electroweak Form Factors of Nucleon and Baryon Resonances

INVESTIGATORS: Hiroshi Ito and Michael J. Musolf (CEBAF and Old Dominion University)

OBJECTIVE: To calculate the electromagnetic and strange form factors of the nucleon and baryon resonances based on the relativistic quark model including the meson cloud corrections.

SIGNIFICANCE: Intermediate-energy neutral current processes involve two classes of physics ${ }^{1}$ : (1) the structure of underlying electroweak gauge theory and (2) hadronic matrix elements of the quark currents. Thus, if we consider the Standard Model of the electroweak interaction to be well-tested, we are able to explore new aspects of hadron structure by using this new probe. In particular, since the strangeness content is nonnegligible in the nucleon ${ }^{2}$ and the neutral current is sensitive to that, one would expect to measure non-vanishing values of the nucleon matrix elements for the strange vector $\left(\bar{s}^{\mu} s\right)$ and axial-vector $\left(\bar{s} \gamma^{\mu} \gamma^{5} s\right)$ currents. The SAMPLE experiment ${ }^{3}$ is underway at MIT/Bates to measure the strange magnetic form factor of the nucleon, $G_{M}^{S}\left(Q^{2}\right)$ at $Q^{2}=0.1(\mathrm{GeV} / \mathrm{c})^{2}$, and the result is expected to give a constraint on the strange magnetic moment of the nucleon, $\mu_{s}=G_{M}^{S}(0)$. The so-called " $G$ " " experiment ${ }^{4}$ is approved at CEBAF to seek the $Q^{2}$-dependence of $G_{M}^{S}\left(Q^{2}\right)$ for $0.1 \leq Q^{2} \leq 0.5(\mathrm{GeV} / \mathrm{c})^{2}$. The parity-violating part of electron scattering ${ }^{5}$ from ${ }^{4} \mathrm{He}$ is expected to determine the strange electric form factor of the nucleon, $G_{E}^{S}\left(Q^{2}\right)$, and the LSND experiment ${ }^{6}$ at LAMPF will measure the form factor of the strange axial-vector current, $G_{A}^{S}\left(Q^{2}\right)$. Whereas these experimental proposals aim to measure the form factors at $Q^{2} \neq 0$, most of the theoretical predictions ${ }^{7}-11$ are made only for $Q^{2}=0$, and are based on effective hadronic degrees of freedom. Meanwhile, the approximate dipole behavior observed in the electromagnetic form factors, for example $G_{E}^{p}\left(Q^{2}\right)$ and $G_{M}^{p}\left(Q^{2}\right)$, can be well reproduced within the relativistic constituent quark model of light-cone dynamics ${ }^{12}$, for momentum transfers $Q^{2} \leq 3(\mathrm{GeV} / \mathrm{c})^{2}$. Thus, by using this successful quark model wave function, we are able to predict the strange form factors of the nucleon as well as the other electroweak form factors of baryon resonances, beyond the effective hadronic theory for the first time. The property of light constituent quarks is the essential issue with regard to the nature of their electroweak couplings ${ }^{3}$ and the meson cloud corrections associated with chiral symmetry breaking in quantum chromodynamics (QCD).

DESCRIPTION OF PROGRESS: The energy scale of the chiral symmetry breaking $\left(\Lambda \chi_{S} \sim 1 \mathrm{GeV}\right)$ is not widely separated from the nonperturbative domain of QCD $\left(\Lambda_{Q C D} \sim 250 \mathrm{MeV}\right)$. Accordingly, one may assume the coexistence of Goldstone bosons $(\pi, K, \eta)$ along with the constituent quarks. The chiral quark model ${ }^{14}$ is based on this idea, where the meson clouds induce the electroweak form factors of the constituent quarks ${ }^{15}$. Of special interest is that clouds of $K$-mesons induce the mixing of strangeness in the $u$ (and d) quark through the $u(d) \rightarrow s+K \rightarrow u(d)$ process. Basically, this program will be completed through two steps: (I) calculation of the quark electroweak form factors from the meson loop diagrams and (II) calculation of the matrix elements for the nucleon and baryon resonances. The current progress and accomplishments are 
PROGRESS

(1) By evaluating the $K$-meson loop diagrams, we have obtained the quark form factors of Dirac- and Pauli- type couplings to the strange vector current, $f_{1}^{S}\left(Q^{2}\right)$ and $f_{2}^{S}\left(Q^{2}\right)$ respectively, given by

$$
\left\langle u\left|\bar{s} \gamma^{\mu} s\right| u\right\rangle=f_{1}^{S}\left(Q^{2}\right) \gamma^{\mu}+\mathrm{i} \frac{\sigma^{\mu \nu} q_{\nu}}{2 M} f_{2}^{S}\left(Q^{2}\right),
$$

where $f_{2}^{S}(0)$ is the strange anomalous moment of $u$-quark, $f_{1}^{S}(0)=0$, and $M$ is the constituent quark mass. These form factors are the same for the $d$-quark.

(2) A similar calculation has been repeated to obtain the quark form factors for the electromagnetic current, $f_{1}\left(Q^{2}\right)$ and $f_{2}\left(Q^{2}\right)$, where $f_{1}(0)=1$ and $f_{2}(0)$ is the anomalous magnetic moment of the constituent quark. The calculation includes the evaluation of many loop diagrams induced by the entire octet $(\pi, K, \eta)$, and each of these contributions is analyzed separately.

(3) With the quark form factors obtained in (1) and (2), we have calculated the electric and magnetic form factors of the nucleon, $G_{E}^{p(n)}\left(Q^{2}\right)$ and $G_{M}^{p(n)}\left(Q^{2}\right)$, and the strange electric- and magnetic- form factors, $G_{E}^{s}\left(Q^{2}\right)$ and $G_{M}^{s}\left(Q^{2}\right)$.

We found that the quark anomalous magnetic moment, $f_{2}(0)$, makes a very significant contribution to the neutron electric form factor, $G_{E}^{n}\left(Q^{2}\right)$, and to the mean square charge radius. The latter observable vanishes in the nonrelativistic quark model. The strange magnetic moment of the nucleon, $G_{M}^{s}(0)$, would vanish if the strange anomalous moment, $f_{2}^{S}(0)$, were absent. The present approach predicts $G_{M}^{s}(0)=-0.110(-0.146)$ with $\Lambda=0.8 \mathrm{GeV}(1.2 \mathrm{GeV})$ for the cut-off mass of meson loops, and the mean square strangeness radius is found to be $\left\langle r_{s}^{2}\right\rangle=-1.89 \times 10^{-2} \mathrm{fm}^{2}\left(-2.31 \times 10^{-2}\right)$. The result for the neutron charge radius is $\left\langle r_{n}^{2}\right\rangle=-0.143 \mathrm{fm}^{2}(-0.082)$, while the experimental value ${ }^{16}$ is $\left\langle r_{n}^{2}\right\rangle=-0.117 \pm 0.002 \mathrm{fm}^{2}$. A paper has been submitted for journal publication ${ }^{17}$, and another including the $Q^{2}$-dependence of the form factors is in preparation. One oral presentation ${ }^{18}$ has been made at an international conference.

By collaborating with Mike Musolf, I expect to complete the following projects within the next three-year period:

(1) Calculation of the nucleon form factor for the strange axial-vector current, $\bar{s} \gamma^{\mu} \gamma^{5} s$.

(2) Calculation of the $\mathrm{N} \rightarrow \Delta$ transition form factor induced by the axial vector current. An experimental proposal ${ }^{19}$ is approved to measure this form factor through the $e+p \rightarrow e^{\prime}+\Delta^{++}+\pi^{-}$reaction at CEBAF.

(3) Investigation of the $\omega-\phi$ mixing within the present framework.

${ }^{1}$ M.J. Musolf, T.W. Donnelly, J. Dubach, S.J. Pollock, S. Kowalski, and E. J. Beise, CEBAF preprint \#TH-93-11.

${ }^{2}$ T.P. Chen and R. Dashen, Phys. Rev. Lett. 26,594(1971); J.F. Donoghue and C.R. Nappi Phys. Lett. 168B,105(1986); J. Gasser, H. Leutwyler and M.E. Sainio, Phys. Lett. 253B, 252(1991).

${ }^{3}$ MIT/Bates proposal 89-06, R. McKeown and D. H. Beck, contact people.

${ }^{4}$ CEBAF proposal PR-91-017, D.H. Beck, spokesperson.

${ }^{5}$ CEBAF proposal PR-91-004, E.J. Beise, spokesperson; CEBAF proposal PR-91-010, J.M Finn and P.A. Souder, sporkespersons.

${ }^{6}$ LSND collaboration, LAMPF proposal \#1173, Los Alamos National Laboratory report 
LA-UR-89-3764 (1989), W.C. Louis, contact person.

'R.L. Jaffe, Phys. Lett. 229B,275(1989).

${ }^{8}$ B.R. Holstein, in Proceedings of the Workshop on Parity Violation in Electron Scattering, E.J. Beise and R.D. McKeown, Eds., World Scientific (1990).

${ }^{9}$ M.J. Musolf and M. Burkardt, CEBAF Theory Preprint \#TH-93-01.

${ }^{10}$ T.D. Cohen, H. Forkel and M. Nilsen, Phys. Lett. 316B,1(1993).

${ }^{11}$ W. Koepf, E.M. Henley and S.J. Pollock, Phys. Lett. 288,11(1992).

${ }^{12}$ P.L. Chung and F. Coester, Phys. Rev. D44,229(1991); L. A. Kondratyuk and M.Terent'ev, Yad. Fiz. 31.1087(1980); I. G. Aznauryan, A. S. Bagdasaryan and N.L.Ter-Isaakyan, Yad.Fiz. $\underline{36}, 1278(1982)$.

${ }^{13} \mathrm{~S}$. Weinberg, Phys. Rev. Lett. 67,3473(1991); ibid., 65,1181(1990).

${ }_{14}^{14} \mathrm{~A}$. Manohar and H. Georgi, Nucl. Phys. B234,189(1984).

${ }^{15}$ D.B. Kaplan and A. Manohar, Nucl. Phys. B310,527(1988).

${ }^{16} \mathrm{~L}$. Koester, W.Nistler and W.W.Waschkowski, Phys. Rev. Lett. 36,1021(1976); G.Höhler et. al., Nucl. Phys. B114,505(1976).

${ }^{17} \mathrm{H}$. Ito, submitted to Phys. Lett. B.

${ }^{18} \mathrm{H}$. Ito, to be published in Proceedings of the 14th International IUPAP Conference on Few-Body Problems in Physics (Williamsburg, VA, May 1994).

${ }^{19}$ CEBAF proposal PR-94-005, L. Elouadrhiri, D.P. Heddle, R. Hicks and $\mathrm{Zh}$. Li, spokespersons; L. Elouadrhiri et. al., University Massachusetts preprint. 
PROGRESS

TOPIC: Exact Three-Body Calculation of Polarization Observables in ${ }^{1} \mathrm{H}(\overrightarrow{\mathrm{d}}, \gamma)^{3} \mathrm{He}$

INESTIGATORS: A.C. Fonseca (U. of Lisbon) and D.R. Lehman

OBJECTIVE: The ultimate scope of this program is to generate theoretical predictions for the key observables in $\vec{d}+p(n) \vec{\leftarrow} \gamma+{ }^{3} \mathrm{He}\left({ }^{3} \mathrm{H}\right)$ from 'realistic' nucleon-nucleon interactions like the Paris, Bonn, and Argonne potentials. Specifically, exact Faddeev calculations (boundstate and continuum) are being performed including the E1 and E2 operators and all components of the ground-state wave function connected through these operators to the allowable continuum states.

SIGNIFICANCE: Attainment of the above objective will permit us to elucidate the mechanism of the E2 rescattering effect and to examine D-state effects in the capture process through the data available on the polarization observables.

DESCRIPTION OF PROGRESS: During the period of this progress report, a large amount of progress was made on this project while Professor Lehman was on sabbatical leare at Duke University (1January 1993 to 30 June 1993). For a six-week period in February and March of 1993, Dr. Fonseca was also present at Duke University, so total focus on this work during that time was possible. At the time of writing our progress report for 1992, we indicated that a code had been developed to handle arbitrary-rank separable interactions. for the above radiative capture reactions. We spent a fair amount of time in the Fall of 1992 checking the code with the EST expansions of the Paris interaction ${ }^{1}$. We confirmed during the spring of 1993 our earlier tentative conclusions that the convergence of the tensor analyzing powers (TAPs) with the rank of the separable expansion is very rapid. Then, in spring 1993, we began our production runs on the Florida State University CRAY for the TAPs over the full energy range of the available experimental data. The outcomes of this work have been described in a short article that has been published in Physical Review $\mathrm{C}$ as a Rapid Communication ${ }^{2}$. Wie are currently drafting a longer paper with more details of our work that will be submitted to Few-Body Systems.

In summary, the purpose of the most recent work on this project was to obtain results for a full Faddeev calculation of the radiative capture of polarized deuterons on protons over the whole energy range of available data for one representative "realistic" NN interaction ( $\mathrm{Paris}$ in this case) ${ }^{3}$. The Paris interaction is handled by the EST expansion method in order to reduce the two-variable integral equations to a single variable. The electromagnetic operator is limited to E1, but this is not a serious limitation in that the measurements fall in the domain where the E1 operator dominates for the TAPs. Since we have already explained and justified the power of the EST expansion method in our earlier proposal and progress report write-ups, it won't be repeated here. We only indicate that the NN interaction is present in both the initial continuum state and the final three-nucleon bound state in the ${ }^{1} \mathrm{~S}_{0},{ }^{3} \mathrm{~S}_{1}-{ }^{3} \mathrm{D}_{1},{ }^{1} \mathrm{P}_{1},{ }^{3} \mathrm{P}_{0},{ }^{3} \mathrm{P}_{1}$, and ${ }^{3} \mathrm{P}_{2}$ partial waves. The ground-state wave function has all possible L-S configurations that can be generated from these NN partial waves and the nucleon-deuteron initial state has all possible orbital angular momentum values consistent with total angular momentum and 
PROGRESS

parity of $J=\frac{1}{2}^{-}$and $\frac{3}{2}^{-}$, where the latter are the only states that can connect to the ground state through the E1 operator.

What becomes clear from our overview of the radiative capture data over this fairly wide energy range (deuteron energies from 10 to $95 \mathrm{MeV}$ ) is that precision data, both statistically and systematically, will be needed for discrimination of theory from experiment. This conclusion is reached by looking at the results ${ }^{4-6}$ for $E_{d}=10,19.8$ and $9.5 \mathrm{MeV}$ with the high precision data points ${ }^{7}$ at 29.2 and $45.3 \mathrm{MeV}$. Furthermore, when we compare our earlier simple rank-1 separable interaction results with the Paris interaction results, it appears that it may be difficult to distinguish between different $\mathrm{NT}$ interactions. This raises the whole issue as to whether there really is sensitivity to the different $N N$ interactions in the TAPs. Maybe, since they are ratios of amplitudes, such differences can be suppressed.

In a global sense, we can say that up to $\mathrm{E}_{d} \sim 30 \mathrm{MeV}$, the TAPs data can be well explained with a theory that involves only E1 radiation, NN partial waves through $\mathrm{P}$ waves, and the NN interaction obtained from the Paris potential. However, above $\sim 30$ $\mathrm{MeV}$, it appears that higher multipoles (at least $\mathrm{E} 2$ ) must be added to the theory. Moreover, we found that the NN P-waves have different significance in different energy regions. Though the Paris interaction does a reasonable job of describing the TAPs orer the full energy range, the simple rank-1 separable models do reasonably well also. However, we have to keep in mind that the differential cross section and the vector analyzing power generally are not reproduced by the present E1 theory. At this stage, we take this latter disagreement to originate from the limitation to E1 radiation as opposed to a shortcoming of the Paris interaction. Clearly, these latter issues indicate the importance of extending the present calculations to include at least the E2 multipole as originally planned, and possibly the M1 multipole as well.

Much progress has been made this spring 1994 towards carrying out calculations with the Bonn $A$ and Bonn $B$ interactions. Preliminary results indicate that there are some differences between the Paris and Bonn results on the level of $10 \%$ in the tensor analyzing powers.

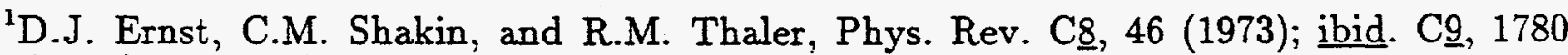
(1974).

2 A.C. Fonseca and D.R. Lehman, Phys. Rev. C48, R503(1993).

${ }^{3}$ M. Lacombe, B. Loiseau, J.M. Richard, R. Winh Mau, J. Côté, P. Pirès, R. DeTourreil, Phys. Rev. C21, 861 (1980).

${ }^{4}$ F. Goeckner, W.K. Pitts, and L.D. Knutson, Phys. Rev. C45, R2536 (1992).

${ }^{5}$ M.C. Vetterli, et al., Phys. Rev. Lett. 54, 1129 (1985).

${ }^{6} W^{-}$.K. Pitts, et al., Phys. Rev. C37, 1 (1988).

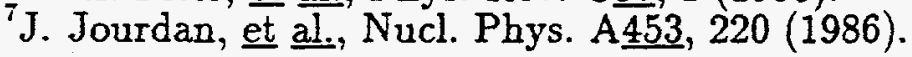


TOPIC: The Quadrupole Moment and the Ratio of the D-wave to S-rave asymptotic normalization constants of ${ }^{6} \mathrm{Li}$

INVESTIGATORS: J.P. Woloschek (former Ph.D. Student) and D.R. Lehman

OBJECTIVE: To explain the quadrupole moment of ${ }^{6} \mathrm{Li}$ within the framework of three-body models.

SIGNIFICANCE: The quadrupole moment of ${ }^{6} \mathrm{Li}$ is one of its most difficult properties to predict. Its small negative value seems to imply, at least within the context of effective two-body $(\alpha-\mathrm{d})$ models, a subtle interplay between the S- and D-wave components of the ${ }^{6} \mathrm{Li}$ wave function. Successful prediction of this observable should lead to a deeper understanding of the $\mathrm{D}$-wave component of the ${ }^{6} \mathrm{Li}$ wave function.

DESCRIPTION OF PROGRESS: One aspect of our previous work ${ }^{1}$ on the elastic electromagnetic form factors of ${ }^{6} \mathrm{Li}$ was to extract the quadrupole moment from the slope of the quadrupole form factor as the electron momentum transfer goes to zero. Unfortunately, this turned out to be a numerically difficult problem due to the fact that the expressions for the form factors are five-fold integrals. Nevertheless, it appeared that the three-body models predict a positive quadrupole moment of $\approx 0.5 \mathrm{e}-\mathrm{fm}^{2}$ in contrast to the experimental value of $-0.0644 \pm 0.0007 \mathrm{e}-\mathrm{fm}^{2}$. To get a better grasp of this result, the alpha-deuteron-component contribution to the quadrupole moment was extracted from the three-body model. When only the alpha-deuteron contribution is present, the ${ }^{6} \mathrm{Li}$ quadrupole moment essentially originates from two terms: 1.) The intrinsic quadrupole moment of the deuteron reduced by the fraction of alpha-deuteron component in the three-body wave function; 2.) An interference contribution that originates from the possibility of either s-wave or $d$-wave relative motion between the alpha-particle and deuteron. In the three-body models, both these terms are positive! However, it has been argued recently, ${ }^{2,3}$ on the basis of alpha-deuteron cluster models, that the interference contribution must be negative. For this alpha-deuteron-projected term to be negative in the three-body models, the s-wave and $\mathrm{d}$-wave effective alphadeuteron wave functions must have opposite signs, especially at large distances. This is not the case; in particular, the s-wave and $d$-wave alpha-deuteron asymptotic normalization constants are of the same sign. As a consequence, it emphasizes the importance of having a reliable experimental determination of the d-wave alphadeuteron asymptotic norm, relative to the s-wave, to check this prediction and indicate the plausibility of the alpha-deuteron cluster-model explanations of the ${ }^{6} \mathrm{Li}$ quadrupole moment. ${ }^{5}$ Nevertheless, one must keep in mind that the three-body wave functions also have a contribution coming from the alpha-(correlated np pair in the continuum) projection that makes up $35-40 \%$ of the norm.

As a consequence of the last point in the previous paragraph, we first carried out a standard calculation of the ${ }^{6} \mathrm{Li}$ quadrupole moment, i.e., directly from the quadrupole operator expectation value with the original three-body ground-state wave functions. The aim was to confirm the results extracted from the quadrupole form factor extrapolations. We found that the previous results are confirmed, i.e., the quadrupole moment does turn out to be positive with magnitude $\approx 0.4 \mathrm{e}-\mathrm{fm}^{2}$. Next, we improved the representation of the ${ }^{3} \mathrm{~S}_{1}-{ }^{3} \mathrm{D}_{1}$ np interaction used in generating the three-body ground-state wave function. The $\mathrm{np}$ interaction underlying the original three-body ${ }^{6} \mathrm{Li}$ 
wave function is that of Yamaguchi-Yamaguchi ${ }^{4}$ which is known to give a poor representation of the mixing parameter $\epsilon_{1}$ and the wrong sign for the barred D-wave phase shift. Our improvement was to allow for an arbitrary-rank separable interaction so that an interaction that gives a reasonable representation of $\epsilon_{1}$ can be used. Interestingly, even with an excellent representation of the np interaction, the EST expansion of the Paris potential, ${ }^{6}$ the quadrupole moment value is predicted to be positive with a magnitude of approximately $\approx 0.53 \mathrm{e}-\mathrm{fm}^{2}$. (Similar results have been announced recently by Shellingerhout, et al., in three-body calculations ${ }^{7}$.) In addition, the s-wave and d-wave alpha-deuteron asymptotic normalization constants retain the same sign though their values change somewhat compared to the original models. Thus, the inadequacy of existing three-body models in explaining the quadrupole moment of ${ }^{6} \mathrm{Li}$ cannot be attributed to the simplicity of the original np interaction. On further reflection and backed up by our new calculations, this should not be surprising. Our new calculations with the Paris interaction indicate that the bulk of the quadrupole-moment value is already given by the alpha-deuteron-projected piece, i.e., approximately $80 \%$. Thus, since the main component of the effective interaction between the alpha and deuteron comes from the alpha-nucleon interaction folded with the deuteron wave function, it immediately becomes clear that the np interaction enters at a secondary level.

Based on the above conclusions, we next looked at the question of inelasticity effects in the alpha-nucleon interaction. On the basis of a set of separable, but coupled-channel, alpha-nucleon interactions originally developed by Miyagawa, et al. ${ }^{8}$ we extended the three-body model to handle these more sophisticated interactions. The new alphanucleon interactions couple to a single hybrid deuteron-triton channel with a threshold at $18.4 \mathrm{MeV}$. With such an approach, the alpha-nucleon phase shifts and inelasticities are reproduced up to nucleon energies of approximately $60 \mathrm{MeV}$, whereas our original alpha-nucleon interactions reproduced the phase shifts up to $20 \mathrm{MeV}$ and ignored the inelasticities since the alpha-particle was considered to be elementary. Moreover, in the new interactions, some of the partial-wave couplings are intriguing from the viewpoint of the quadrupole moment and the angular momenta involved. For example, the alphanucleon ${ }^{2} \mathrm{~S}_{1 / 2}$ partial. wave couples to the deuteron-triton ${ }^{4} \mathrm{D}_{1 / 2}$ partial wave. In addition, we have now included the ${ }^{2} \mathrm{D}_{3 / 2},{ }^{2} \mathrm{D}_{5 / 2},{ }^{2} \mathrm{~F}_{5 / 2}$, and ${ }^{2} \mathrm{~F}_{7 / 2}$ partial waves of the alpha-nucleon interaction besides the original ${ }^{2} \mathrm{~S}_{1 / 2},{ }^{2} \mathrm{P}_{1 / 2}$, and ${ }^{2} \mathrm{P}_{3 / 2}$ partial waves. Such an approach recognizes the fact that the alpha-particle is not an elementary particle. We find that when the underlying NN interaction is that of the rank-1 YamaguchjYamaguchi case, the D- to S-wave asymptotic-normalization ratio is already negative with only the S- and P-wave alpha-nucleon interactions present. Addition of the ${ }^{2} D_{3 / 2}$ partial wave of the alpha-nucleon interaction to this framework leads to the most negative value of the asymptotic-norm ratio. As the other alpha-nucleon partial waves are added, the asymptotic-norm becomes less negative. However, upon changing the N. interaction to the rank-6 EST expansion of the Paris potential, the asymptoticnorm ratio becomes positive for all alpha-nucleon partial-wave cases considered above. Eren when the asymptotic-norm ratio is negative, the alpha-deuteron contribution to the ${ }^{6} \mathrm{Li}$ quadrupole moment is positive.

The above model gave hope, but is limited in the sense that once the alpha-nucleon 
system makes the transition to the "deuteron-triton" channel, there are no interactions between the 'spectator' nucleon and either the deuteron or triton. As a result, threebody-force effects are not generated. The so-called 'dispersive' effect of the channel coupling is present, but no three-body-force component appears. As a consequence. most recently, we constructed a rank-1, coupled-channel interaction where the alphanucleon system can make a transition to a pseudo-alpha-particle state that we call alpha* with the same threshold as indicated above. Our hope was that such a model would not only have the 'dispersive' effect of the channel coupling, but that the interaction would not preclude generation of three-body-force contributions. While the investigation of the presence or absence of three-body-force contributions was underway, results for the asymptotic normalization ratio and the full ${ }^{6} \mathrm{Li}$ quadrupole moment calculation were obtained. The results for the asymptotic norm ratio are similar to the -deuteron-triton" channel-coupling model, while the values for the alpha-deuteron component of the ${ }^{6} \mathrm{Li}$ quadrupole moment move to smaller values. The values for the full calculation of the ${ }^{6} \mathrm{Li}$ quadrupole moment are significantly reduced compared to the model where no channel-coupling is present, but they are not negative. Meanwhile, an analysis of the issue of three-body-force contributions in coupled-channel problems where the interaction is rank-1 has demonstrated that the equations can be written in a form that appears to eliminate any such contributions. If this conclusion is correct, it appears that full three-body-force-effect contributions require at least rank-2 in the channel coupling.

The results of these three different levels of calculation to understand the ${ }^{6} \mathrm{Li}$ quadrupole moment will be described in a series of papers to be submitted for publication.

A. Eskandarian, D.R. Lehman, and W.C. Parke, Phys. Rev. C38,2341(1988).

A.C. Merchant and N. Rowley, Phys. Lett. 150B,35(1985).

${ }^{3}$ T. Mertelmeir and H.M. Hofmann, Nucl. Phys. A $\underline{459}, 387(1986)$.

Y. Yamaguchi and Y. Yamaguchi, Phys. Rev. 95,1635(1954).

${ }^{3}$ R. Crespo, A.M. Eiro, and F.D. Santos, Phys. Rev. C 399,305 (1989).

'M. Lacombe, B. Loiseau, J.M. Richard, R. Winh Mau, J. Côté, P. Pirès, R. DeTourreil, Phys. Rev. C21,861(1980). J. Haidenbauer and W. Plessas, Phys. Rev. C30, 1822 (1984); ibid., C32,1424(1985); J. Haidenbauer, Y. Koike, and W. Plessas, Phys. Rev. C $33,439(1986)$.

'N.W. Shellingerhout, L.P. Kok, S.A. Coon, and R.M. Adam, Phys. Rev. C48 $2714(1993)$.

${ }^{E}$ K. Miyagawa, Y. Koike, T. Ueda, T. Sawada, and S. Takagi, Prog. Theor. Phys. 74, 1264(1985).

'D.R. Lehman, to be submitted for publication. 
PROGRESS

TOPIC: Angular Distribution Coefficients for $(\gamma, \mathrm{X})$ Reactions with Circularly Polarized Photons and Polarized Targets

INIESTIGATORS: H.R. Weller and R.M. Chasteler (Duke Lniversity), B.S. Marks (N.C. State), R.G. Seyler (Ohio State), and D.R. Lehman

OBJECTIVE: To present in convenient form the formalism for the case of linearly or circularly polarized photons on polarized targets.

SIGNIFICANCE: Presentation of the formalism for incident linearly or circularly polarized photons on polarized targets allows one to generate expressions for cross sections and analyzing powers in terms of reduced matrix elements. Such equations are extremely useful in planning experiments with an aim towards extracting specific physically observable effects.

DESCRIPTION OF PROGRESS: Our earlier work in this area dealt with the angular momentum formalism of linearly polarized photons in $(\gamma, \mathrm{X})$ reactions on unpolarized targets ${ }^{1}$. The main intention of the present work is to investigate the general formalism of Welton ${ }^{2}$ for the case of linearly or circularly polarized photons on polarized targets, to verify his results in detail, put them into a convenient form, and illustrate their use by an example. One of the strongly motivating factors behind this work is the recent interest in the Drell-Hearn-Gerasimov (DHG) sum rule ${ }^{3}$. Direct experimental test of the DHG sum rule will require measurements employing circularly polarized photons and polarized proton targets. In re-deriving Welton's expressions, we discovered an error in a phase factor, which affects the Tables of Ref. 1. This correction to Welton's equations has been carefully verified and is explained in our paper to appear in Atomic Data and Nuclear Data Tables.

${ }^{1}$ H.R. Weller, J. Langenbrunner, R.M. Chasteler, E.L. Tomusiak. J. Asai, R.G. Seyler, and D.R. Lehman, Atomic Data and Nuclear Data Tables 50,29 (1992).

${ }^{2}$ T.A. Welton in Fast Neutron Physics, edited by J.B. Marion and J.L. Fowler (Interscience, New York, 1963), Vol. II, p. 1317.

$3 \mathrm{~J}$. Soffer and O. Teryaev, Phys. Rev. Letts. 70,3373 (1993).

${ }^{4}$ H.R. Weller, R.M. Chasteler, B.S. Marks, R.G. Seyler, and D.R. Lehman, Atomic Data and Nuclear Data Tables $\underline{52}$, in press (1994). 
PROGRESS

TOPIC: Long-range behavior of the effective nucleon-deuteron interaction for the three-nucleon system

INTESTIGATORS: D.R. Lehman, L.C. Maximon, Walid Younes (former undergraduate physics major at GW, now graduate student at Rutgers U.), and B.F. Gibson (Los Alamos National Laboratory)

OBJECTIVE: The aim of this work is to derive rigorously the asymptotic behavior of the effective interaction between a nucleon and deuteron within the bound three-nucleon system.

SIGNIFICANCE: One goal in attempts to understand the low-energy properties of the three-nucleon system is to determine whether these properties can be understood within the framework of two-body models with effective interactions. Until recently, such twobody models have met with no success. However, Tomio, Delfino, and Adhikari, ${ }^{1,2}$ recently published an analysis of the low-energy properties of the three-nucleon system based on an ad-hoc effective two-body interaction that has an attractive $e^{-\mu \mathrm{r}} / \mathrm{r}^{2}$ longrange tail which is essential for the success of the description ( $r$ is the separation distance between the center-of-mass of the deuteron and the neutron). In particular, their effective, three-parameter, potential is found to predict correctly the value of the doublet $p-d$ scattering length obtained from exact three-body calculations and to reproduce the correlation between the doublet scattering length and the three-nucleon binding energy found in exact three-body calculations for both the n-d and p-d systems, after having been fit to the experimental values for the ${ }^{3} \mathrm{H}$ and ${ }^{3} \mathrm{He}$ binding energies and the $n-d$ doublet scattering length. In an attempt to justify the long-range behavior of the $n$-d effective interaction used by Tomio et al. ${ }^{1,2}$, Delfino, Frederico, and Tomio. hare derived the asymptotic behavior starting from a zero-range representation of the three-nucleon bound-state wave function. The essence of their approach is to project the bound-state wave function with a nucleon-deuteron state (freely moving relative to each other) and then substitute this resulting two-body wave function into the two-body Schrödinger equation to derive an effective potential that would lead to the assumed form of the effective $n-d$ wave function within ${ }^{3} \mathrm{H}$. They find by this approach that the effective $n-d$ potential at large separation distances behaves as $-e^{-\lambda r} / \mathrm{r}^{3 / 2}$. Though their result differs from that of Tomio et al ${ }^{1,2}$, they argue that it is similar in that both contain the qualitative feature of depending on a range associated with the size of the deuteron (through $\mu$ and $\lambda$ ). They also argue that their result must be valid for any short-range interaction since it is based on minimal assumptions about the three-nucleon wave function, i.e., a zero-range form. However, it is particularly this assumption about the form of the three-nucleon wave function which casts doubt on the reliability of their conclusions. It is well known that in zero-range approximation, the three-nucleon system has infinite binding energy. ${ }^{4}$ Historically, it was this fact that led the early workers on the two-nucleon interaction to the conclusion that the two-nucleon interaction has a short, but finite, range. ${ }^{5}$ Therefore, it is important to attempt another approach towards deriving the asymptotic behavior of the effective $n$-d potential in the three-nucleon system.

It should be noted, however, that Tomio, Frederico, and Delfino ${ }^{6}$ do extend their 


\section{PROGRESS}

approach to a more general assumed form for the three-nucleon bound-state wave function. They extend their zero-range model to include a parameter that, in some sense, accounts for the finite-range of the two-nucleon interaction. This parameter is set by requiring reproduction of the triton binding energy and the doublet $n-d$ scattering length with the deuteron binding energy set at its experimental value. This time, the problem of finding the effective $n-d$ potential must be approached numerically. They argue that their numerical results justify the phenomenological potential of Tomio. Delfino, and Adhikari. ${ }^{1,2}$

DESCRIPTION OF PROGRESS: The approach taken in the current work is to begin from the three-body Schrödinger equation for the bound-state wave function without making specific assumptions about the two-nucleon interactions (possible three-nucleon interactions are neglected). The equation is projected with a bra state composed of a nucleon plus deuteron moving freely relative to each other (no detailed form for the deuteron wave function is assumed). The aim is to obtain an equation for this effective $n$-d wave function within ${ }^{3} \mathrm{H}$, i.e., for the overlap amplitude of the $n-d$ state and the three-nucleon bound state. Except for the two terms containing the potentials in the coordinates that involve the 'external' nucleon and one of the nucleons in the deuteron, this falls out directly. In the latter 'permuted-potential' terms, a complete set of nucleon-correlated-pair states is inserted to affect the sought after equation. (The correlated-pair terms are the deuteron and an $n-p$ scattering state.) It is then immediately apparent that we must deal with a coupled set of effective two-body equations, one corresponding to the effective nucleon-deuteron wave function and the other to the effective nucleon--n-p--continuum wave function. By iterative substitution, the effective nucleon--n-p--continuum wave function can be eliminated from the equation for the effective $n$ - $d$ wave function at the expense of having an infinite series of terms containing successively increasing powers of the two-nucleon interaction. In the effective $n-d$ ware function equation, the leading term of the effective interaction is local (assuming the two-nucleon interactions are local), while the succeeding terms are nonlocal. These latter terms involve, in the simplest case, a Green's function with the tro-nucleon interaction on either side. Initially, we completed a thorough study of the local part of the effective interaction.

Our purpose in investigating the local part of the $n-d$ effective potential was to determine its long-range behavior. The local part of the effective potential is constructed from a matrix element of the 'permuted-potential' terms between deuteron wave functions, sometimes called a 'folding potential'. We started with specific potentials that were analytically tractable (s-waves only): 1. Zero-range; 2 . Squarewell; and 3. Hulthén. We found that the square-well and Hulthén potentials lead to an asymptotic behavior of - (constant) $e^{-4 \gamma \mathrm{r}} / \mathrm{r}^{2}$, where the binding energy of the deuteron defines $\gamma$, i.e., $\mathrm{B}_{\mathrm{d}}=\gamma^{2} / \mathrm{M}$. Moreover, the 'constant' multiplying the asymptotic form is given in terms of the potential parameters. Based on these analyses, it was then decided to assume an arbitrary short-range two-nucleon interaction, e.g., with Yukawa behavior at large separation distances, to see if the result can be made potential independent. The answer is that it can. The final expression has the above form with the constant being determined by a particular integral over the potential. The general result has been checked with the specific cases listed above and the previously obtained results have been reproduced. Interestingly, when we start with the 
assumption of a nonlocal two-nucleon interaction given by the standard Yamaguchi ${ }^{7} \mathrm{~s}$ ware form, we find an asymptotic behavior of - (constant) $e^{-(2 \gamma+\alpha) r} / r$, where $B_{t}-B_{d}=3 \alpha^{2} / 4$, in general agreement with the conclusions of Fonseca, Redish, and Shanley concerning a Yukawa form. ${ }^{8}$ At least at the level of the local part of the $n-d$ effective potential, there appears to be a difference in the asymptotic behavior depending on the nature, local or nonlocal, of the underlying two-nucleon interaction.

What has been learned from this investigation so far? First, and most importantly, we found for the zero-range interaction that the local-part of the effective interaction is identically zero. Since it is expected, from our initial study so far, that the nonlocal contribution to the effective potential falls off differently and possibly more rapidly asimptotically than the local piece, and since the authors of Ref. 3 neglected the nonlocal contribution, this demonstrates that the ansatz used by these authors is invalid. Secondly, if our tentative conclusion about the asymptotic behavior of the nonlocal piece is correct, our result explicitly substantiates the ad hoc assumption of Tomio et al. ${ }^{1}{ }^{12}$ as to the asymptotic behavior of the effective $n$-d potential. Moreover, the precise parameter to be used in the exponential is obtained. It differs in numerical value significantly (approximately a factor of 4 larger) from that found by the fits in Refs. 1 and 2. The magnitude of the potential in the asymptotic region is explicitly given by the multiplicative constant. Thirdly, our asymptotic result and the exact expression for the potential can be used in combination to find at what separation distance the asymptotic behavior begins to dominate. This has been done numerically.

In addition to the above, we have been investigating, so far without a definitive conclusion, the question of the Efimov limit in the above framework. The Efimov limit is the situation where $\gamma$ approaches zero and $r$ is large but less than $1 / \gamma$. In this limit, we would expect to find that the interaction approaches a $1 / \mathrm{r}^{2}$ behavior -- essential for the explanation of the Efimov effect. ${ }^{9}$ Our work on this question and the nature of the succeeding nonlocal corrections to the local part of the effective $n-d$ interaction was minimal this year owing to higher priorities. However, by methods reminiscent of the Feshbach projection operators in reaction theory ${ }^{10}$, in spring 1993 it was found possible to rewrite the infinite series on the right-hand side of the equation for the effective nucleon-deuteron wave-function in a more compact form. This new form looks potentially more suitable to analysis for the Efimov effect questions.

${ }^{1}$ L. Tomio, A. Delfino, and S.K. Adhikari, Phys. Rev. C쪼, 441 (1987).

${ }^{2}$ S.K. Adhikari, A. Delfino, and L. Tomio, in Proceedings of the International Workshop on Few-Body Approaches to Nuclear Reactions in Tandem and Cyclotron Energy Regions, edited by S. Oryu and T. Sawada (World Scientific, Singapore, (1987), p52.

3 A. Delfino, T. Frederico, and L. Tomio, Phys. Rev. C $\underline{8}, 11$ (1988).

${ }^{4}$ L.H. Thomas, Phys. Rev. 47, 903 (1935).

${ }^{5}$ H.A. Bethe and R.F. Bacher, Rev. Mod. Phys. 8,82 (1936).

${ }^{6} \mathrm{~L}$. Tomio, T. Frederico, and A. Delfino, Phys. Rev. C411, 876 (1990).

${ }_{8}^{7} Y$. Yamaguchi, Phys. Rev. 95, 1628 (1954).

${ }^{8}$ A.C. Fonseca, E.F. Redish, and P.E. Shanley, Nucl. Phys. A $\underline{320}, 273$ (1979).

${ }^{2} \mathrm{~V}$. Efimov, Nucl. Phys. A362, 45 (1981).

${ }^{10}$ H. Feshbach, Ann. Phys. (N.Y.) 19, 287 (1962). 
PROGRESS

IOPIC: Production of E1 Radiation in the ${ }^{2} \mathrm{H}(\mathrm{d}, \gamma){ }^{4} \mathrm{He}$ Reaction at Very Low Energies INVESTIGATORS: D.R. Lehman, H.R. Weller, and R.M. Whitton

OBJECTIVE: To show that the mechanism of charge polarization of the deuterons in the reaction ${ }^{2} \mathrm{H}(\mathrm{d}, \gamma)^{4} \mathrm{He}$ cannot give rise to $\mathrm{E} 1$ radiation owing to the identity of the target and projectile.

SIGNIFICANCE: Recent experimental results ${ }^{1}$ suggest that over $50 \%$ of the cross section in the ${ }^{2} \mathrm{H}(\mathrm{d}, \gamma)^{4} \mathrm{He}$ reaction below $\mathrm{E}_{d}=80 \mathrm{keV}$ arises from $\mathrm{P}$-wave capture. Analysis of the data implies that this strength arises from El radiation. In this work, we consider possible origins of this $\mathrm{E} 1$ radiation and show that its origin must arise from the spin-dependent isoscalar part of the E1 operator.

DESCRIPTION OF PROGRESS: Our work begins by assuming that the ${ }^{2} \mathrm{H}(\mathrm{d}, \gamma){ }^{4} \mathrm{He}$ reaction contains a large P-wave capture component which leads to E1 radiation at $E_{d}=80 \mathrm{keV}$. This is at first sight a surprise, since isovector $E_{1}$ transitions are known to be forbidden in self-conjugate nuclei if $\Delta T=0$. Since the $d+d$ channel has $T=0$, as does the ${ }^{4} \mathrm{He}$ ground state, the El radiation in this reaction should be isoscalar. The question then arises as to possible origins of $\mathrm{E} 1$ radiation in the ${ }^{2} \mathrm{H}(\mathrm{d}, \gamma){ }^{4} \mathrm{He}$ reaction. Since the entrance channel is composed of identical bosons, we must have $L+S$ even so that the $1^{-}$state (which is needed to generate E1 radiation to the $0^{+}$ground state of ${ }^{4} \mathrm{He}$ ) must have $\mathrm{L}=1$ and $S=1$. If the ground state of ${ }^{4} \mathrm{He}$ is purely $\mathrm{L}=0, \mathrm{~S}=0$, the $\mathrm{E} 1$ transition must arise from the spin-dependent isoscalar part of the E1 operator. However, another possible mechanism exists: Charge polarization of the deuterons. This would correspond to a specific mechanism of isospin mixing due to the Coulomb force. It arises because of the fact that the charge of the deuterons does not reside at their respective centers-of-mass, but rather on the proton within each deuteron. Thus, one deuteron in the Coulomb field of the other deuteron has a preferential orientation oring to the induced electric-dipole moment. Since the resulting state must have a mixed parity, symmetry requires that it have a mixed isospin. The two fused deuterons could thereby form a state of ${ }^{4} \mathrm{He}$ which would have a $\mathrm{T}=1$ component, and isovector El radiation could evolve.

In this work, we show that the mechanism of deuteron charge polarization cannot produce $\mathrm{E} 1$ radiation in the ${ }^{2} \mathrm{H}(\mathrm{d}, \gamma)^{4} \mathrm{He}$ reaction. This is so because the polarizationpotential matrix element vanishes identically for the ${ }^{4} \mathrm{He}$ reaction owing to the identity of the projectile and target nuclei. This is not the case for ${ }^{2} \mathrm{H}(\alpha, \gamma){ }^{8} \mathrm{Li}$, because the initial state contains unlike particles. Therefore, so long as isospin is conserved, any EIradiation produced in this reaction must be due to the spin-dependent part of the E1 operator. A manuscript describing this work has been submitted to Few-Body Systems ${ }^{2}$.

${ }^{1}$ L.H. Kramer, et al., Phys. Lett. B304, 208 (1993).

${ }^{2}$ D.R. Lehman, H.R. Weller, and R.M. Whitton, "Production of E1 Radiation in the ${ }^{2} \mathrm{H}(\mathrm{d}, \gamma){ }^{4} \mathrm{He}$ Reaction at Very Low Energies, submitted to Few-Body Systems. 


\section{PROGRESS}

TOPIC: Approximate Ways to Treat the Nucleon-Nucleon Tensor Force in the FourNucleon Bound State

INTESTIGATORS: A.C. Fonseca (U. of Lisbon), B.F. Gibson (LANL), and D.R. Lehman

OBJECTIVE: To show how to include as much of the nucleon-nucleon tensor force as possible in the dominant S-state component of the four-nucleon wave function for the purpose of optimizing the starting point in the iterative solution of the full four-body problem.

SIGNIFICANCE: Until the initial work of Fonseca $^{1}$ and the more recent work of Kamada and Glöckle ${ }^{2}$, the two-nucleon tensor force in the four-nucleon system had always been treated approximately ${ }^{3-6}$, leading to four-nucleon bound-state equations in which all relevant orbital angular momenta are zero. As a consequence, the resulting four-nucleon wave function is reduced to the $\varphi=0, \mathcal{L}=0$ components with symmetric and mixed-symmetric spatial configurations. At the time, the question was "how close to the exact four-nucleon binding energy can these approximate methods lead us?" The purpose of this work is to answer that question for several approximation methods tested in the four-nucleon bound-state problem as means to understand how the twonucleon tensor force propagates through the underlying $(2)+(2)$ and $(3)+1$ subsystem amplitudes to yield a final four-nucleon binding energy.

DESCRIPTION OF PROGRESS: This work has been completed and a manuscript has been published in Physical Review C. $^{7}$ Since the various approximation methods discussed in the manuscript are rather tedious to delineate, suffice it to say that of the six approximations considered for handling the nucleon-nucleon tensor force in the fourbody problem, two turn out to be of good quality and essentially equivalent. Of these two. the so-called $t_{00} / t_{00}$ approximation becomes the recommended one owing to its simplicity. The $t_{00}$ approach was first initiated by John $\mathrm{Tjon}^{4}$ in the three-body problem and amounts to use of the full two-body $t$-matrix truncated to the $l=l^{\prime}=0$ element in the three-body equations. The $t_{00} / t_{00}$ approximation in the four-body problem has the same starting point and is applied in both the $(2)+(2)$ and $(3)+1$ subamplitudes of the four-body equations. The aim would be first to generate the fourbody wave function and binding energy in the $t_{00} / t_{00}$ approximation and then use that ware function and binding energy as the starting point for solving the full four-body equations without approximation. The latter approach gives an almost optimal starting point in comparison to starting with an arbitrary initial wave function and a guess at the starting binding energy.

${ }^{1}$ A.C. Fonseca, Phys. Rev. C440, 1390 (1989).

${ }^{2}$ H. Kamada and W. Glöckle, Nucl. Phys. A548, 205 (1992); N.R. Shellingerhout, J.J. Schut, and L.P. Kok, Phys. Rev. C4ㅌ, 1192 (1992).

${ }^{3}$ B.F. Gibson and D.R. Lehman, Phys. Rev. C14, 685 (1976); ibid., C15, 2257E (1977); ibid., C18, 1042 (1978).

${ }^{4}$ J.A. Tjon, Phys. Rev. Lett. 40, 1239 (1978).

${ }^{5}$ S. Sofianos, H. Fiedeldey, and H. Haberzettl, Phys. Rev. C222, 1772 (1980).

${ }^{6}$ A.C. Fonseca, Few-Body Systems 1,69 (1986).

'A.C. Fonseca, B.F. Gibson, and D.R. Lehman, Phys. Rev. C4ㄹ, 2528(1993). 
PROGRESS

TOPIC: Neutron Halo Few-Body Nuclei

INTESTIGATORS: Mohamed Najmeddine (Ph.D. Student solely supported by grant) and D.R. Lehman (Director)

OBJECTIVE: To apply few-body methods to those neutron-halo nuclei that naturally cluster into three- or four-body systems in order to study their structure, and weak and electromagnetic interaction properties.

SIGNIFICANCE: It is now known ${ }^{1}$ that ${ }^{6} \mathrm{He}$ and ${ }^{11} \mathrm{Li}$ exbibit large neutron halos or dilute neutron skins (long neutron tails extending well outside the nucleus), especially

${ }^{11} \mathrm{Li}$. Experiments that make it possible to measure interaction cross sections and/or reaction cross sections at a range of energies for light radioactive nuclei provide data on the nuclear sizes of these neutron-rich nuclei. The above two nuclei exhibit halo-type structure with two loosely bound valence neutrons and only one bound state. They are interesting as three-body systems in that none of the two-body systems within the three-body framework are themselves bound. Therefore, the opportunity exists to study true three-body features associated with the neutron-halo structure.

DESCRIPTION OF PROGRESS: Mr. Najmeddine accompanied Professor Lehman during his sabbatical leave at Duke University. During this period, Mr. Najmeddine spent about $50 \%$ of his time learning about techniques and methods in few-body physics and about $50 \%$ studying the literature concerning neutron-halo nuclei. The purpose of this effort was for Mr. Najmeddine to define a research problem for his Ph.D. dissertation. He is now investigating and working on two problems concerning neutronhalo nuclei: 1) Development of a three-body model of ${ }^{11} \mathrm{Li}$ using the latest experimental information on the $\mathrm{n}^{9}{ }^{9} \mathrm{Li}$ system to develop a good phenomenological interaction for this two-body subsystem. 2) Initiating a complete (full three-body dynamics for both the bound and continuum states) three-body calculation of ${ }^{6} \mathrm{He} \beta$ decay into the $\alpha$-deuteron continuum. The aim for the first calculation is to use the ware function obtained to examine the neutron momentum distributions within these nuclei compared to experimental data and to calculate their neutron radii. In the second item, it is believed ${ }^{2}$ that this beta decay process should be sensitive to the neutron-halo structure of ${ }^{6} \mathrm{He}$ because the Gamow-Teller transition will emphasize overlap between the deuteron in the $\alpha$-deuteron continuum and the di-neutron configuration in ${ }^{6} \mathrm{He}$. However, since the measured values for the branching-ratio momentum distribution are very small, it will be essential to handle the few-particle dynamics of the reaction fully before information about the neutron-halo structure of ${ }^{6} \mathrm{He}$ can be extracted. Existing calculations ${ }^{2}$ do not treat the $\alpha$-deuteron continuum as an exact eigenfunction of the three-body Hamiltonian and this might be the source of their over-estimate of the data by an order of magnitude.

${ }^{1}$ M.V. Zhukov, B.V. Danilin, D.V. Fedorov, J.M. Bang, I.J. Thompson, and J.S. Vaagen, "Bound State Properties of Borromean Halo Nuclei: ${ }^{6} \mathrm{He}$ and ${ }^{11} \mathrm{Li}^{\prime}$, to be published in Physics Reports, Nordita Preprint-92/90 N, and references therein.

${ }^{2}$ M.V. Zhukov, B.V. Danilin, L.V. Grigorenko, and N.B. Shul'gina, Phys. Rev. C $47,2937(1993) ;$ A. Csoto and D. Baye, Phys. Rev. C $499,813(1994)$. 
PROGRESS

TOPIC: Evaluation of the Spherical Bessel Transform of a Whittaker Function; An Application of a Difference Equation Method

\author{
ITESTIGATOR: L.C. Maximon
}

OBJECTIVES: To obtain a simple analytic expression for the spherical Bessel transform of the bound state Coulomb wave function for the $\ell$-th partial wave, expressed as a Whittaker function. To derive a method for treating a wide class of integrals of similar form, based only on the existence of differential-difference equations for the functions involved, and the difference and differential equations satisfied by the integral.

SIGNIFICANCE: Integral transforms (Bessel, Hankel, Fourier, etc.) of functions which are the solution of wave equations (in general, second order differential equations) occur in almost any analysis of quantum mechanical problems. The method developed provides both analytic expressions for such transforms and recurrence relations which lend themselves readily to evaluation by computers. Since it relies essentially only on the differential and difference equations satisfied by the functions involved, it is applicable to a wide class of problems. This work was undertaken in support of the experimental effort at TUNL, conducted by E. Ludwig of UNC and R. Das at Duke. They used a low energy (7-10 MeV) deuteron beam on a heavy target, investigating the pickup reactions $\mathrm{d}+\mathrm{n} \rightarrow{ }^{3} \mathrm{H}, \mathrm{d}+\mathrm{p} \rightarrow{ }^{3} \mathrm{He}$, and $\mathrm{d}+\mathrm{d} \rightarrow{ }^{4} \mathrm{He}$ to obtain cross sections and analyzing powers, providing an experimental test of model wave functions. The method dereloped for obtaining integral transforms was applied in particular to the spherical Bessel transform of the bound state Coulomb wave function for the $\ell$-th partial wave, expressed as a Whittaker function. This result was needed for the analysis of the experiments, providing an analytic expression for the effective $n-d$ and $p-d$ wave functions in momentum space, including the Coulomb interaction, for small momenta. This was required, in particular, for $\ell=0$ and $\ell=2$.

DESCRIPTION OF PROGRESS: This project has been completed; the work was published in the Journal of Mathematical Physics $\underline{33}, 2005$ (1992). 
PROGRESS

TOPIC: Computer Program for Landau Straggling

INTESTIGATORS: L.C. Maximon and D.P. Heddle (Christopher Newport College

OBJECTIVE: To write and make available for use by the community engaged in high and medium energy electron scattering experiments, a fast and accurate code for the computation of Landau straggling. This code will incorporate the best expressions available for the electron-electron interaction (Møller scattering) within the basic formulation given originally by Landau. The documentation accompanying this code will include a detailed consideration of the approximations involved in this formulation with references to relevant articles, together with a discussion of possible modifications for an improved version.

SIGNIFICANCE: The accuracy of the nuclear information obtained from high energy electron scattering measurements carried out with the new generation of electron accelerators depends on and is often limited by the theoretical uncertainties in the radiative corrections which must be applied to the experimental data. If useful nuclear information is to be obtained from high energy coincidence measurements, then the radiative corrections must be known theoretically at the one percent level. Landau straggling is one of these radiative corrections; this project is one element in the overall program of providing state of the art expressions for all of the radiative corrections.

DESCRIPTION OF PROGRESS: This project was completed; the work was published in Computer Physics Communications $70,77(1992)$. 
PROGRESS

TOPIC: User's Guide to Radiative Corrections

INTESTIGATOR: Leonard C. Maximon

OBJECTIVE: To present, in an easily accessible form, a review of the state of the art expressions for the various radiative corrections which must be applied to high-energy electron scattering data.

SIG.NIFICANCE: The ability of experimenters to extract accurate information on nuclear and nucleon structure from measurements using high energy continuous-wave electron accelerators depends critically on the possibility of making the necessary radiative corrections to the measured electron scattering data. It is essential that the analysis of data include the most accurate radiative corrections currently available and that there is an understanding of the limitations placed on our knowledge of nuclear structure because of uncertainties in the radiative corrections.

DESCRIPTION OF PROGRESS: The final form of the current version of the User's Guide has been determined. It is expected that this project will be completed in calendar year 1995. It will consist of sections on each of the radiative corrections applied to electron scattering measurements, viz., Landau straggling, thick-target (external) bremsstrahlung, and the Schwinger radiative correction. It will review the state of the art expressions for each of these corrections, including, in each case, a list of the references considered relevant to providing the user with the source of the expressions as well as material deemed useful pedagogically. The sections on Landau straggling and thick-target bremsstrahlung are essentially complete. The section on the Schwinger radiative correction is currently being reworked to simplify the derivation of the expressions which appear in the literature and make the derivation accessible to the non-specialist. A review of the guide by experimentalists at CEBAF has been planned and will be undertaken before submitting the final form. 
PROGRESS

TOPIC: High-Energy Approximations for the Total Born Cross Section for Pair Production in a Screened Coulomb Field

\section{INVESTIGATOR: Leonard C. Maximon}

OBJECTIVE: To derive a high-energy limit of the momentum distribution associated with the total Born-approximation cross section for pair production that is free of the large cancellations inherent in the exact distribution.

SIGNIFICANCE: One of the fundamental cross sections in photonuclear research is the total cross section for electron-positron pairs produced in the nuclear field. The currently available momentum distribution is unsuitable for integration at high energies because of large cancellation of individual terms in the distribution. The high energy limit of this distribution is free of such cancellation and is useful for numerical evaluation of the total cross section including the effects of atomic screening.

DESCRIPTION OF PROGRESS: This project has been completed; the work was published in Phys.Rev.C $\underline{49}, 428(1994)$. 
PROGRESS

TOPIC: $\alpha+\mathrm{d} \rightarrow{ }^{6} \mathrm{Li}+\gamma$ Scattering Cross Section and Polarization Observables at Low Energies

NTESTIGATORS: W.C. Parke and Ana Eiro (U. of Lisbon)

OBJECTIVE: To calculate the low-energy cross section and polarization observables for the reaction $\alpha+d \rightarrow{ }^{6} \mathrm{Li}+\gamma$ using consistent three-body dynamics in both the initial and final states.

SIG.NIFICANCE: Understanding the radiative capture of alpha particles on deuterons (or deuterons on alpha particles) has importance in astrophysics, nuclear physics, and nuclear technology. The capture reaction ${ }^{2} \mathrm{H}(\alpha, \gamma)^{6} \mathrm{Li}$ at low incident energies has been a focus of interest to both astrophysicists and nuclear physicists. ${ }^{1}$ The value of the cross section below center-of-mass energies of about $2 \mathrm{MeV}$ is important in determining the abundance of ${ }^{6} \mathrm{Li}$ from the standard big-bang model and from cosmic ray generation. Most recently, it has been emphasized by Rolfs and Barnes in their review of astrophysically important radiative capture reactions that the reaction mechanism remains uncertain. ${ }^{2}$ With polarized deuterons, or for the inverse photodisintegration process, polarized photons, the reaction may uncover information about the internal structure of the ${ }^{6} \mathrm{Li}$ nucleus. The reaction is also important in a controlled-fusion plasma. The analysis of the produced gammas can be used to determine the plasma temperature. ${ }^{3}$

Measurements at center-of-mass energies from 1 to $8 \mathrm{MeV}$ have been made, showing a cross section between 4 and 100 nanobarns which seems to be dominated by an E2 direct capture mechanism. ${ }^{4}$ Another series of measurements closer to the reaction threshold has been proposed. ${ }^{5}$

Besides the cross-section measurement, there has been recent interest in using radiative capture of polarized deuterons on the alpha particle $e^{5,6}$ to get a handle on the D-state component in ${ }^{6} \mathrm{Li}$, in particular, on the ratio of the $\mathrm{D}$ to $\mathrm{S}$ asymptotic normalization constants for ${ }^{6} \mathrm{Li} \rightarrow \alpha+\mathrm{d}$, as well as the difficult-to-predict electric quadrupole moment. Experiments at Duke (TUNL) studying the reaction ${ }^{4} \mathrm{He}(\mathbf{d}, \gamma){ }^{6} \mathrm{Li}$ are pending. ${ }^{5}$

As yet, there is no fundamental and fully consistent calculation of the radiative capture reaction, although several effective two-body calculations have been published. ${ }^{6}$ A three-body (alpha-particle, neutron, and proton) calculation for both the incoming and outgoing nuclear wave functions, using the same two-body interactions in the threebody scattering state and in the bound state, would satisfy self-consistency. This approach has already been successful ${ }^{7}$ in the case of the calculation of the two-body photodisintegration of ${ }^{3} \mathrm{H}$ and ${ }^{3} \mathrm{He}$, where effective two-body models have not worked. Moreover, using a three-body wave function in the initial state would automatically include the ${ }^{6} \mathrm{Li}\left(3^{+}\right)$resonance state in the $\alpha-\mathrm{d}$ continuum. This state enhances the reaction cross section by almost two orders of magnitude near threshold (at about 700 $\mathrm{keV}$ in the center-of-mass). Such an approach should provide the sound footing necessary to delineate the reaction mechanism of this radiative capture reaction.

DESCRIPTION OF PROGRESS: We have completed the derivation of the full threebody radiative capture amplitude, with the bound-state and incoming scattering states 


\section{PROGRESS}

both determined br the same three-body Hamiltonian. Coding is underway. We will be able to compare with the contributions of initial-state rescattering, within a fully consistent effective two-body framework (no effective two-body calculations to date satisfy such consistency), and with the full three-body calculation to see the effects of three-body dynamics. Our effective two-body Born term results already show that the contribution to the reaction cross section can be sensitive to the quadrupole moment of the deuteron, particularly at center-of-mass energies (near $30 \mathrm{MeV}$ ) which probe the radial node in the $\alpha-\mathrm{d}$ wave function (this node coming from the underlying Pauli exclusion principle for the nucleons in the two clusters). We have also calculated the tensor analyzing power $T_{20}$. As expected, this polarization observable is controlled by the D-state components in the $\alpha-\mathrm{d}$ system, with the deuteron quadrupole term being the dominant contributor near $90^{\circ}$, even at center-of-mass energies of $4 \mathrm{MeV}$. Our calculation will give a clean way to test the contribution of continuum NN states in the intermediate rescattering process.

${ }^{1}$ D.N, Schramm and R.V. Wagoner, Annu. Rev. Nucl. Sci. 27,37(1977); R.G.H. Robertson et. al.. Phys. Rev. Lett. 47,1867(1981); K. Langanke, C. Rolfs, Z. Phys. A $\underline{325}, 193(1986)$.

${ }^{2}$ C. Rolfs and C.A. Barnes, Annu. Rev. Nucl. Part. Sci. 40,45(1990).

${ }^{3} \mathrm{E}$. Cecil, private communication.

${ }_{5}^{4}$ Q.K.K. Liu, H. Kanada, and Y.C. Tang, Phys. Rev. C $\underline{33}, 1561(1986)$.

${ }^{5} \mathrm{H}$. Weller, private communication.

${ }^{6} \mathrm{~K}$. Langanke, Nucl. Phys. A 457,351(1986); K. Langanke and C. Rolfs, Z. Phys. A 325,193(1986); R. Crespo, A.M. Eiro, and F.D. Santos, Phys. Rev. C39,305(1989); R. Crespo, A.M. Eiro, and J.A. Tostevin, Phys. Rev. C42,1646(1990).

${ }^{7}$ B.F. Gibson and D.R. Lehman, Phys. Rev. C11,29(1975); ibid 13.477(1976); A.C. Fonseca and D.R. Lehman, Phys. Lett. B267,159(1991), A.C. Fonseca and D.R. Lehman, Phys. Rev. C쑈,R503(1993). 
PROGRESS

TOPIC: Accurate Triton Wave Function by a New Separable Expansion Method

INVESTIGATORS: Y. Koike (Hosei University, Tokyo), D.R. Lehman, L.C. Maximon and W.C. Parke

OBJECTIVE: To test the new expansion method of Koike for realistic three-body systems.

SIGNIFICANCE: Efficient separable expansion methods will allow more sophisticated and accurate three- and four-body calculations for bound state and scattering processes.

DESCRIPTION OF PROGRESS: We have demonstrated that a clear convergent series for the three-body observables is obtained with a new separable expansion method where several techniques are unified. In the method, we utilize both the separable expansions of EST and of Adhikari-Sloan, ,, 2 splitting the original potential into two potentials with different ranges. The Adhikari-Sloan method is re-formulated in a simple manner to show the possibility of a separable expansion more clearly. The method has been applied to the AV14 potential as an example. The binding energy and s-. p-, and d-wave probabilities for the triton have been calculated, as shown below, compared with the Los Alamos local-potential calculation. ${ }^{3}$ This work shows that a separable expansion is both a reliable and a very efficient method among all possible methods of "rigorous" three-body calculations.

$\begin{array}{lclll}\text { rank } & \text { B.E. }(\mathrm{MeV}) & \mathrm{P}\left(\mathrm{S}^{\prime}\right) & \mathrm{P}(\mathrm{P}) & \mathrm{P}(\mathrm{D}) \\ & & & & \\ 1-1 & 7.635 & 1.349 & 0.0465 & 8.084 \\ 3-4 & 7.440 & 1.292 & 0.0747 & 8.857 \\ 4-7 & 7.437 & 1.357 & 0.0729 & 8.862 \\ 6-9 & 7.441 & 1.363 & 0.0741 & 8.859 \\ 6-12 & 7.443 & 1.362 & 0.0746 & 8.856 \\ 8-16 & 7.442 & 1.364 & 0.0746 & 8.856 \\ 10-20 & 7.442 & 1.364 & 0.0746 & 8.855 \\ \text { Los Alamos } & 7.441 & 1.36 & 0.08 & 8.86\end{array}$

${ }^{1} J$. Haidenbauer and W. Plessas, Phys. Rev. C27,63(1983), J. Haidenbauer and Y. Koike, Phys. Rev. C34,1187(1986).

${ }^{2}$ W.C. Parke, Y. Koike, D.R. Lehman and W.C. Maximon, Few-Body Systems $311,89(1991)$.

${ }^{3}$ C.R. Chen, G.L. Payne, J.L. Friar and B.F. Gibson, Phys. Rev. C31,2266(1985). 
PROGRESS

TOPIC: A Possible Treatment of Three-Body Bound States with Long-Range Interactions

INTESTIGATORS: Y. Koike (Hosei University, Tokyo), D.R. Lehman, L.C. Maximon and W.C. Parke

OBJECTIVE: To formulate an effective technique for handling long-range interactions in bound states, including Coulomb effects in the three-nucleon system.

SIGNIFICANCE: The inclusion of long-range forces in bound and scattering problems can be mathematically difficult due to the possibility that the long-range part of the interaction may not decrease fast enough to allow convergence of expressions for certain observables. Various methods have been tried in the past. ${ }^{1,2.3}$ A new scheme for handling infinite-range interactions in the bound state is proposed.

DESCRIPTION OF PROGRESS: Coupled integral equations have been derived for the bound-state three-body problem with long-range two-body interactions like the Coulomb interaction. We take advantage of the fact that the ware function is localized in the bound state to define physical amplitudes. The two-body Lippmann-Schwinger equation and the three-body Faddeev-Schrödinger equations are converted into equations for these amplitudes for the bound-state case, which are well defined even for long-range interactions. Introducing base functions which preserve the physicallycorrect threshold and asymptotic behavior, we can derive one-dimensional coupled integral equations which have the same structure as the standard three-body bound state equation, with higher rank separable interactions. We will apply these techniques to ${ }^{3} \mathrm{He}$ and ${ }^{6} \mathrm{Li}$.

${ }^{1}$ E.O. Alt, W. Sandhas, and H. Ziegelmann, Phys. Rev. C17,1981(1978).

${ }^{2}$ D.R. Lehman, A. Eskandarian, B.F. Gibson, and L.C. Maximon, Phys. Rev. C $229,1450(1984)$.

${ }^{3}$ A.C. Fonseca and M.T. Peña, Phys. Rev. A $\underline{38,4967(1988) .}$ 


\section{PROPOSED WORK BY TOPIC}

Following this separator page are the descriptions of proposed work by topic. Each item has a layout similar to the progress reports, i.e., Topic, Investigators, Objective, Significance, and Description of Proposed Work. In addition to the work that is described, work will continue on several projects that were described in the progress section and for which we felt it was not necessary to elaborate any further. Most of these investigations are expected to be completed within the next eighteen months. Those projects that are not further described in this section, but constitute proposed (continued) work are the following:

TOPIC:

D-State Structure of ${ }^{4} \mathrm{He}$

INVESTIGATORS: H. Ito, H. Haberzettl, D.R. Lehman, and B. F. Gibson (LANL). Progress Report p. 51

TOPIC:

Electroweak Form Factors of Nucleon and Baryon Resonances

INVESTIGATORS: Hiroshi Ito and Michael J. Musolf (CEBAF and Old Dominion University)

Progress Report p. 56

TOPIC: $\quad$ Exact Three-Body Calculation of Polarization Observables in ${ }^{1} \mathrm{H}(\overrightarrow{\mathrm{d}}, \gamma)^{3} \mathrm{He}$

INVESTIGATORS: A.C. Fonseca (U. of Lisbon) and D.R. Lehman

Progress Report p. 59

TOPIC:

The Quadrupole Moment and the Ratio of the $D$-wave to S-wave asymptotic normalization constants of ${ }^{6} \mathrm{Li}$

INVESTIGATORS: J.P. Woloschek (former Ph.D. Student) and D.R. Lehman

Progress Report p. 61

TOPIC:

Long-range behavior of the effective nucleon-deuteron interaction for the three-nucleon system

INTESTIGATORS: D.R. Lehman, L.C. Maximon, W. Younes (former undergraduate physics major at GWU, now graduate student at Rutgers U.), and B.F. Gibson (Los Alamos National Laboratory)

Progress Report p. 65

TOPIC:

Neutron Halo Few-Body Nuclei

INVESTIGATORS: Mohamed Najmeddine (Ph.D. Student solely supported by grant) and D.R. Lehman (Director)

Progress Report p. 70

TOPIC:

Accurate Triton Wave Function by a New Separable Expansion Method

INVESTIGATORS: Y. Koike (Hosei University, Tokyo), D.R. Lehman, L.C. Maximon and W.C. Parke

Progress Report p. 77 
TOPIC:

A Possible Treatment of Three-Body Bound States with LongRange Interactions

INVESTIGATORS: Y. Koike (Hosei University, Tokyo), D.R. Lehman, L.C. Maximon and W.C. Parke

Progress Report p. 78 
PROPOSED

TOPIC: Photon- and Pion-Induced Eta Production on the Nucleon

INVESTIGATORS: R. Pratt (Ph.D. Student), C. Bennhold, and L. Tiator (U. of Mainz, Germany)

OBJECTIVE: To construct a covariant and unitary coupled-channels model that can describe $\eta$ production with photons and pions consistently.

SIGNIFICANCE: In contrast to pions, which excite the $\Delta$ (with isospin $I=3 / 2$ ) and $N^{*}(I=1 / 2)$ resonances simultaneously, the $\eta$-meson, due to its isoscalar nature, can be employed to selectively probe $\mathrm{N}^{*}$ states. In the low energy regime, there is significant preference for intermediate excitation of the $S_{11}(1535)$ state in the $\eta \mathrm{N}$ system which has not yet been understood in quark models. $\mathrm{A}$ successful description of the resonance sector in the $\gamma \mathrm{N} \rightarrow \eta \mathrm{N}$ reaction should permit the extraction of the $\eta \mathrm{NN}$ coupling constant, which via $\eta-\eta^{\prime}$ mixing is related to the $\mathrm{U}_{A}(1)$ anomaly of $\mathrm{QCD}$ and the axial flavor singlet coupling measured by recent EMC data. A coupled channels framework that simultaneously describes the $\pi \mathrm{N}$ and $\eta \mathrm{N}$ channels will allow us to extract the controversial $\eta \mathrm{N}$ scattering length, whose magnitude may determine whether eta-mesic bound states can exist.

DESCRIPTION OF PROPOSED WORK: The proposed work is a continuation of an existing project. ${ }^{1}$ Previous studies on this subject performed a number of approximations in the development of an amplitude for the $(\gamma, \eta)$ process. Ref. 2 constructed a non-relativistic isobar model whose resonance couplings were fitted to pion scattering and photoproduction. The Born terms were added separately ${ }^{3}$, which violates unitarity. In other models, ${ }^{4}$ the $(\gamma, \eta)$ data were used for a direct fit to resonance parameters; thus, the relationship to the pion channels was never established.

The proposed work aims at correcting these flaws by constructing a fully covariant and unitary coupled channels framework that can simultaneously describe the hadronic processes $\pi \mathrm{N} \rightarrow \pi \mathrm{N}, \pi \mathrm{N} \rightarrow \eta \mathrm{N}$, and $\eta \mathrm{N} \rightarrow \eta \mathrm{N}$ as well as the electromagnetic production of pions and etas, $\gamma \mathrm{N} \rightarrow \pi \mathrm{N}$ and $\tilde{\gamma} \mathrm{N} \rightarrow \eta \mathrm{N}$. Our starting point will be the model dereloped in Ref. 5 for $\pi \mathrm{N} \rightarrow \pi \mathrm{N}$ and $\gamma \mathrm{N} \rightarrow \pi \mathrm{N}$. In this model, $\pi \mathrm{N}$ scattering up to $\mathrm{T}_{\pi}$ $=600 \mathrm{MeV}$ is described by a manifestly covariant wave equation, in which the pion is restricted to its mass shell. The kernel of the equation includes the nucleon, $\mathrm{P}_{11}(1440)$, $\mathrm{D}_{13}(1520)$, and $\Delta_{33}(1232)$ poles, along with contact $\sigma$ - and $\rho$-like exchange terms. Chiral symmetry is maintained at threshold. The resonance contributions are fully unitarized by the equation, with their widths determined by the dynamics of the model. The resonance parameters are then adjusted to reproduce the $\pi \mathrm{N}$ phase shifts. As a first step, we will increase the energy range of the model by including the $S_{11}$ (1535) state - the most important resonance to which the $\eta \mathrm{N}$-system couples at low energies. Furthermore, we will develop a coupled channels framework that will involve all possible $\pi \mathrm{N}$ and $\eta \mathrm{N}$ channels. This is important since the $\mathrm{S}_{11} \pi \mathrm{N}$ phase shift actually reveals a cusp effect at the $\pi \mathrm{N} \rightarrow \eta \mathrm{N}$ threshold. Refitting the resonance parameters to the $\pi N$ phase shifts will enable us to predict, rather than fit, the $\pi^{-} p \rightarrow \eta n$ reaction.

In a second step, this hadronic model will be used to describe final state interactions in the $(\gamma, \pi)$ and $(\gamma, \eta)$ processes. Using the $(\gamma, \pi)$ multipoles to constrain the $\gamma \mathrm{NN}^{*}$ vertices, we will again be able to predict the $\gamma \mathrm{N} \rightarrow \eta \mathrm{N}$ reaction. On the other hand, 
using the forthcoming, high-quality Mainz data, we can check for consistency by using the $(\gamma, \eta)$ data to extract resonance parameters and then to compare these with the results obtained from the fits to the $(\gamma, \pi)$ data. Ultimately, we plan to extend this model to the $S_{11}(1650)$ and $P_{11}(1710)$ region and to $\eta$ electroproduction.

${ }^{1}$ See Progress Report, project "Photo- and Electroproduction...", p. 23.

${ }^{2}$ C. Bennhold and H. Tanabe, Nucl. Phys. A $\underline{50}, 625(1991)$; Phys. Lett. B243,13(1990); Lect. Notes in Phys. 365,190 (1990).

${ }^{3} \mathrm{~L}$. Tiator, C. Bennhold, S.S. Kamalov, Nucl. Phys. A, in press, (1994).

${ }_{5}^{4} \mathrm{M}$. Benmerrouche and N.C. Mukhophadyay, Phys. Rev. Lett. 67,1070(1991).

${ }^{5} F$. Gross and Y. Surya, Phys. Rev. C447,703(1993). 
PROPOSED

TOPIC: Exclusive Quasifree $\eta$ Photoproduction on Nuclei

INVESTIGATORS: Xiaodong Li (Ohio U.), L. E. Wright (Ohio U.), C. Bennhold, and L. Tiator (U. of Mainz, Germany)

OBJECTIVE: To study the $\eta$-nucleus optical potential and to search for modifications of the $S_{11}$ in the nuclear medium.

SIGNIFICANCE: Quasifree $\eta$ photoproduction on nuclei allows one to study the behavior of the $S_{11}(1535)$ resonance in the nuclear medium. These medium modifications are especially interesting in comparison to the modifications of the $\Delta$ properties that are introduced by the $\Delta$-hole model. Furthermore, this reaction is sensitive to the $\eta$-nucleus final state interaction. A strong attractive potential may lead to the presence of the exotic eta-mesic bound nuclear states, which would show up in the $(\gamma, \mathrm{p})$ excitation function just below the $\eta$ production threshold.

DESCRIPTION OF PROPOSED WORK: The proposed work is a continuation of an existing project. ${ }^{1}$ To extend our formalism ${ }^{2}$ from pions to etas, we will need to exchange the production amplitude and the optical potential of the pion with those of the eta. We will use the $(\gamma, \eta)$ operator recently developed ${ }^{3}$ and perform a t $\rho$ approximation for the $\eta$-nucleus optical potential. The $\eta \mathrm{N} \rightarrow \eta \mathrm{N}$ t-matrix will be taken from a coupled channels isobar analysis ${ }^{3}$ that uses $\pi \mathrm{N}$ data to constrain the $\eta \mathrm{NN}^{*}$ vertices. The results will be compared to forthcoming data from Mainz.

${ }^{1}$ See Progress Report, project "Photo- and Electroproduction...", p. 23.

${ }^{2}$ Xiaodong Li, L.E. Wright and C. Bennhold, Phys. Rev. C $48,816(1993)$.

${ }^{3}$ C. Bennhold and H. Tanabe, Nucl. Phys. A530,625(1991); Phys. Lett. B243,13(1990); Lect. Notes in Phys. 365,190(1990); L. Tiator, C. Bennhold, S.S. Kamalov, Nucl. Phys. A. in press, (1994). 
TOPIC: Pion Scattering and Pion Photoproduction on the Deuteron

INVESTIGATORS: S.S. Kamalov (JINR, Dubna, Russia), C. Bennhold, and L. Tiator (U. of Mainz, Germany)

OBJECTIVE: To explore the limits of applicability of the multiple scattering formalism by comparing theoretical predictions with $\pi \mathrm{d} \rightarrow \pi \mathrm{d}$ and $\gamma \mathrm{d} \rightarrow$ $\pi \mathrm{d}$ data and with $\pi$ NN Faddeev approaches.

SIGNIFICANCE AND PROPOSED WORK: The proposed work is a continuation of an existing project. ${ }^{1}$ The coupled channels, multiple-scattering formalism developed for the trinucleon ${ }^{2}$ will be applied to the deuteron in order to investigate whether the deviations seen in reactions on the trinucleon at large $Q$ will also be visible here. Special attention will be paid to polarization observables, especially the vector and tensor analyzing powers. A comparison with more sophisticated $\pi$ NN Faddeev approaches will be made.

${ }^{1}$ See Progress Report, "Pion- and Photon-Induced Reactions on the Trinucleon", p. 30.

${ }^{2}$ S.S. Kamalov, L. Tiator and C. Bennhold, Phys. Rev. C47,941(1993); C. Bennhold et al., Nucl. Phys. A540,621(1992); S.S. Kamalov, L. Tiator, and C. Bennhold, Few Body Systems $10,143(1991)$. 
PROPOSED

TOPIC: The Nonmesonic Weak Decay of Hypernuclei

INVESTIGATORS: C. Bennhold, A. Ramos (U. of Barcelona, Spain), and A. Parreno (Ph.D. student at U. of Barcelona, Spain)

OB.JECTIVE: To extract information on the weak hadronic vertices by analyzing the nonmesonic hypernuclear decay.

SIGNIFICANCE AND PROPOSED WORK: This work is a continuation of a previous project. $^{1}$ We plan to extend our formalism to study possible violations of the $\Delta I=1 / 2$ rule in the $\Lambda \mathrm{N} \rightarrow \mathrm{NN}$ process. In the absence of strong interactions, the lowest-order Hamiltonian for the weak quark-quark interaction is given by the $V$ - $A$ theory with the Cabibbo hypothesis. However, this Hamiltonian leads to both $\Delta \mathrm{I}=1 / 2$ and $\Delta \mathrm{I}=3 / 2$ transitions with comparable strength, in contradiction to experimental data on kaon and free hyperon decays which suggest a ratio of these two amplitudes of about 20 . In order to explain this empirical $\Delta I=1 / 2$ rule, strong interaction corrections to the Cabibbo theory have been suggested. Among these are contributions from renormalization and one-loop gluon radiative corrections, such as penguin diagrams which contribute only to $\Delta \mathrm{I}=1 / 2$ transitions. Recent quark model calculations find large $\Delta \mathrm{I}=3 / 2$ amplitudes for the $\Lambda N \rightarrow N N$ process. ${ }^{2}$ However, these amplitudes have not yet been used in a realistic hypernuclear calculation. We intend to implement these $\Delta I=3 / 2$ quark model amplitudes into our hypernuclear shell-model code to see the real effect these transitions will have on total and partial decay rates.

Furthermore, we plan to investigate all possible polarization observables that could be used experimentally to study the nonmesonic decay. A recently completed experiment at $\mathrm{KEK}^{3}$ measured the asymmetry of the angular distribution of protons coming from the decay of polarized hypernuclei produced via the $\left(\pi^{+}, \mathrm{K}^{+}\right)$reaction. However, a number of other polarization observables are possible for the process $\Lambda N \rightarrow N N$, some of which are well-known ${ }^{4}$ in the study of polarized NN scattering as well as in that of parity-violating weak NN scattering. We intend to systematically look at the sensitivity of these polarization observables and of asymmetry parameters. to the model ingredients.

${ }^{1}$ See Progress Report, "The Nonmesonic Weak Decay of Hypernuclei", p. 27.

${ }^{2} \mathrm{M}$. Ota and K. Maltman, private communications and preprints.

${ }^{3}$ S. Ajimura et al., Phys. Lett. B282,293(1992).

${ }^{4}$ L. Wolfenstein, Ann. Rev. Nucl. Sci. $\underline{6}, 43(1956)$. 
TOPIC: Two-Body Mechanism in Pion and Eta Photoproduction on the Trinucleon

INVESTIGATORS: S.S. Kamalor (JINR, Dubna. Russia), L. Tiator (U. of Mainz, Germany), and C. Bennhold

OBJECTIVE: To study the breakdown of the Impulse Approximation in high momentum transfer reactions on ${ }^{3} \mathrm{He}$ and ${ }^{3} \mathrm{H}$.

SIGNIFICANCE: Reactions on the trinucleon are an ideal testing ground to search for effects that go beyond the Impulse Approximation because realistic correlated threebody wave functions are available that are reliable even at high nuclear momentum transfers. Previous studies ${ }^{1,2}$ of pion scattering and pion photoproduction on the trinucleon systems were mostly based on a multiple-scattering approach. Calculations performed in momentum space allowed a straightforward inclusion of the nonlocalities in the pion-nuclear interaction and an exact treatment of Fermi motion. Within a coupled-channels framework, it has become possible to consistently describe $\pi^{+}$and coherent $\pi^{0}$ photoproduction, as well as elastic and charge-exchange pion scattering on ${ }^{3} \mathrm{He}$ and ${ }^{3} \mathrm{H}$, and to obtain a good description of experimental measurements at low momentum transfers. Thus, the calculations have reached a point where the conventional one-body aspects are treated on a rather accurate level.

However, at higher momentum transfers, large discrepancies appear between measurements and theoretical calculations. In the region of $\mathrm{Q}^{2}>8 \mathrm{fm}^{-2}$, calculations dramatically fail to explain existing ${ }^{3} \mathrm{He}\left(\gamma, \pi^{+}\right)^{3} \mathrm{H}$ data, underestimating them by up to two orders of magnitude. Similar discrepancies were found in elastic pion scattering at backward angles, as well as in pion single charge-exchange and pion-induced eta production on ${ }^{3} \mathrm{He}$ at higher energies.

DESCRIPTION OF PROPOSED WORK: It has been known for a long time that by imposing gauge invariance in electromagnetic reactions with the nucleus one can generate two-body meson exchange currents via the Siegert Theorem. Very recently, this method was applied to the specific reaction of pion photoproduction on the trinucleon. ${ }^{3}$ Using general requirements such as current conservation in the nuclear electromagnetic vertex, the authors of Ref. 3 obtained general expressions for two-body correction terms similar to meson exchange currents in electron scattering. However, this procedure generates only the convection part of the two-body currents since the magnetic parts fulfill gauge invariance separately.

The goal of this work is to study the effects of genuine two-body mechanisms in pion photoproduction on ${ }^{3} \mathrm{He}$ that do not appear in a standard DWIA framework. We demand that these two-body currents be generated in a way that is consistent with the description of pion photoproduction of a single nucleon in the Impulse Approximation. This consistency requirement can be satisfied in a straightforward manner by starting from an effective Lagrangian for the pion-nuclear production process and by introducing the electromagnetic field using minimal substitution. This method not only guarantees gauge invariance but also allows the explicit calculation of contributions from the magnetic moments.

In order to obtain the seagull and pion exchange terms of the usual one-body charged 
pion photoproduction operator, we will employ minimal substitution in the $\pi \mathrm{NN}$-vertex and in the pion wave function. The nucleon pole terms can be generated by introducing the electromagnetic field in the nuclear wave function. It is at this level that, besides the standard one-body s- and u-channel Born terms, new two-body operators will be generated that go beyond the Impulse Approximation. As a last step, these new amplitudes will be used in our multiple scattering formalism in order to include the pion final-state interaction. In a second phase of this project, we are planning to extend this program to the as yet unmeasured ${ }^{3} \mathrm{He}(\gamma, \eta)^{3} \mathrm{He}$ reaction as well as to pion scattering and single charge-exchange at high $\mathrm{Q}$ by including diagrams similar to those added in $\pi$ d elastic scattering ${ }^{4}$.

${ }^{1}$ S.S. Kamalov, L. Tiator, and C. Bennhold, Phys. Rev. C47,941(1993); C. Bennhold et al. Nucl. Phys. A $\underline{540}, 621(1992)$.

${ }^{2}$ S.S. Kamalov, L. Tiator, and C. Bennhold, Few Body Systems 10,143(1991).

${ }^{3}$ A.S. Raskin, E.L. Tomusiak, and J.L. Friar, Few Body Systems, in press, (1994).

${ }^{4} \mathrm{~B}$. K. Jennings, Phys. Lett. $\underline{\mathrm{B} 205}, 187(1988)$. 
PROPOSED

TOPIC: Electromagnetic Production of Kaons on Nucleons and Nuclei

INIESTIGATORS: C. Bennhold, H. Ito, T. Mart (Ph.D. student at the U. of Mainz. Germany), and D. Drechsel (U. of Mainz, Germany)

OBJECTIVE: To describe the reactions $\mathrm{N}(\gamma, K) \Sigma$ and $\mathrm{N}\left(e, \mathrm{e}^{\prime} \mathrm{K}\right) \Sigma$ for all possible isospin channels, to study the $\mathrm{K}^{0}$ and $\mathrm{K}^{*}$ electromagnetic form factors, and to predict hypernuclear production rates.

SIGNIFICANCE: Previous studies have focused solely on the processes $\mathrm{p}\left(\gamma, \mathrm{K}^{+}\right) \Lambda$ and $\mathrm{p}\left(7 . \mathrm{K}^{-0}\right) \Sigma$ since some data have been available for these reactions. However, different model parametrizations, each of them describing the above processes fairly well, produce results for $\mathrm{p}\left(\gamma, \mathrm{K}^{0}\right) \Sigma$ and $\mathrm{n}\left(\gamma, \mathrm{K}^{+}\right) \Sigma^{-}$that overpredict the few available data by orders of magnitude. Thus, in view of the new Bonn data and upcoming, approved CEBAF experiments, studying all of the isospin channels can yield constraints on coupling constants and contributing resonances which are much more stringent than were previously thought. Once coupling constants have been fixed by photoproduction data. the reaction $\left(e, e^{\prime} K^{0}\right)$ can be used to extract the $\mathrm{K}^{0}$ and $\mathrm{K}^{*}$ electromagnetic form factors. Furthermore, the elementary amplitude can be used to predict the rates of hypernuclear photoproduction as well as that of quasifree kaon production.

DESCRIPTION OF PROPOSED WORK: Most analyses of kaon electromagnetic production $^{1-4}$ over the last several years have focused on the two processes $\gamma \mathrm{p}-\mathrm{K}^{+} \Lambda$ and $\gamma \mathrm{p} \rightarrow \mathrm{K}^{+} \Sigma^{0}$, due to the fact that almost all of the few available photokaon data have been taken for these two reactions. In order to further constrain the leading coupling constants, we plan to develop extensions of previous models, including the other four isospin channels. We can then compare the predictions with the few available total cross section data for the charged $\Sigma$-photoproduction reactions, $\gamma \mathrm{p} \rightarrow \mathrm{K}^{0} \Sigma^{+}$and $\gamma \mathrm{n} \rightarrow \mathrm{K}^{+} \Sigma^{-}$. With the advent of the CEBAF Large Acceptance Spectrometer (CLAS) in Hall B at CEBAF, which can not only detect neutral kaons at least as efficiently as $\mathrm{K}^{+}$, but can also measure kaon photoproduction on the neutron using deuterium, a study of the other isospin channels is clearly called for. Furthermore, new data for various $(\gamma, \mathrm{K})$ channels are currently being analyzed in Bonn ${ }^{5}$.

As in previous studies, our model includes the standard Born terms along with the intermediate $\Lambda$ and $\mathrm{K}^{*}$ exchange. Furthermore, we incorporate the $\mathrm{N}^{*}$ resonances $S_{11}(1650)$ and $P_{11}(1710)$ as well as the $\Delta$ resonances $S_{31}(1900)$ and $P_{31}(1910)$ which can onls contribute to $\Sigma$ photoproduction. In order to generalize the $\gamma \mathrm{p} \rightarrow \mathrm{K}^{+} \Lambda$ and $\gamma \mathrm{p}-\mathrm{K}^{+} \Sigma^{0}$ amplitudes to the other four channels, $\gamma \mathrm{n} \rightarrow \mathrm{K}^{0} \Lambda, \gamma \mathrm{p} \rightarrow \mathrm{K}^{0} \Sigma^{+}$, in $-\mathrm{K}^{+} \Sigma^{-}$, and $\gamma \mathrm{n} \rightarrow \mathrm{K}^{0} \Sigma^{0}$, we use isospin symmetry to relate the hadronic vertices among each other, and we use either experimental decay widths or quark model calculations to obtain the additional electromagnetic transition moments. Preliminary calculations indicate that models which give an adequate description of the $\gamma \mathrm{p} \rightarrow \mathrm{K}+\Sigma^{0}$ data can drastically fail to reproduce the measurements of the charged $\Sigma$ channels. This may be due to the magnitude of the Born coupling constants in the different models. We will also determine what additional information can be extracted from the new Bonn data $^{5}$ by including them in our fits of the coupling constants and resonance parameters. 
Once the couplings have been determined in the $(\gamma, \mathrm{K})$ reactions. we will proceed to study electromagnetic form factors in $\left(e, e^{\prime} K\right)$. While the $\mathrm{K}^{+}$form factor has been studied in more detail, that for $\mathrm{K}^{0}$ is largely unknown. Although the $\mathrm{K}^{0}$-meson is electrically neutral, the charge form factor does not vanish for $q^{2} \neq 0$. In the constituent quark model of the $\mathrm{K}^{0}(d \bar{s})$-meson, $F_{K^{0}}$ can be described by two independent form factors, both of which include coupling the photon to a quark: the $d$-quark for the first form factor and the $\bar{s}$-quark for the second one. Their $q^{2}$-dependences are different, except for $q^{2}=0$, because the masses of $u$ - and $s$-quarks are different. and thus $F_{K^{0}}(q)$ has a non-zero value. According to a preliminary calculation within the relativistic quark model, ${ }^{6}$ the results of the $\mathrm{K}^{0}$ and $\mathrm{K}^{+}$form factors can be re-expressed in terms of monopole functions, which are based on the Vector Meson Dominance (VMD) hypothesis. However, this quark-meson duality seems to be valid only for the low- $q^{2}$ region $\left(q^{2}<2 \mathrm{GeV}^{2}\right)$; beyond this region, the quark model form factors deviate significantly. We will investigate the sensitivity of the $n\left(e, e^{\prime} K^{0}\right) \Lambda$ response functions to different $\mathrm{K}^{6}$ form factors.

The amplitude of the $\mathrm{K}^{*}+\gamma \rightarrow \mathrm{K}$ reaction has the structure of the AVV-anomaly, which is the strange-quark counterpart to the $\rho+\gamma \rightarrow \pi$ reaction. This reaction involves a spin-flip of a quark, and from simple power counting of perturbative $\mathrm{QCD}$, the form factor is expected to behave asymptotically like $1 / q^{4}$. Here, again, significant deriations from the VMD result were predicted for the $\rho+\gamma \rightarrow \pi$ case, ${ }^{7}$ but no investigation has been made for the $\mathrm{K}^{*}+\gamma \rightarrow \mathrm{K}$ case. Furthermore, for the $\mathrm{N}\left(\mathrm{e}, \mathrm{e}^{\prime} \mathrm{K}\right) Y$ reaction, most analyses assume a factorization of the form factors, $F_{K^{0}}^{*}(q) \propto$ $F_{K^{+}}^{*}(q)$, where $F_{K^{0}}^{*}(q)$ and $F_{K^{+}}^{*}(q)$ are the transition form factors of the $\mathrm{K}^{0 *}+\gamma \rightarrow$ $\mathrm{K}^{0}$ and $\mathrm{K}^{+*}+\gamma \rightarrow \mathrm{K}^{+}$reactions, respectively. We plan to calculate these form factors within the relativistic quark model defined in the Light-Cone frame. ${ }^{8}$ In addition to predicting these form factors, this calculation will help us to understand better the factorization hypothesis and VMD in the strangeness sector.

Once the elementary amplitude has been obtained to a reasonable degree of accuracy, one can calculate hypernuclear photoproduction. We will focus especially on the reaction ${ }^{3} \mathrm{He}\left(\gamma, \mathrm{K}^{+}\right)_{\mathrm{A}}^{3} \mathrm{H}$, using our expertise from the corresponding ${ }^{3} \mathrm{He}\left(\gamma, \pi^{+}\right)^{3} \mathrm{H}$ and ${ }^{3} \mathrm{He}(\gamma, \eta)^{3} \mathrm{He}$ reactions. The hypertriton with $\mathrm{B}_{\mathrm{A}}=0.13 \mathrm{MeV}$ is the lightest bound nuclear system containing strangeness. Its production with pion or kaon beams requires either a ${ }^{3} \mathrm{H}$ target or involves neutral mesons in the final state. We will compute cross sections for this reaction by first using a simple ${ }^{3} \mathrm{H}$ wave function that is based on a simple model ${ }^{9}$ in which the $\Lambda$ is weakly bound to a deuteron. Using the elementary amplitude derived before, the relevant matrix elements will be evaluated in momentum space to include nonlocalities and Fermi motion. Since the $\mathrm{K}^{+}$interaction is rather weak on a hadronic scale, the $\mathrm{K}^{+}$final state interaction will be neglected. As a second step, we will study sensitivites to details in the production operator and to different hypertriton wave functions.

${ }^{1}$ R.A. Adelseck and B. Saghai, Phys. Rev. C42,108(1990).

${ }^{2} \mathrm{H}$. Tanabe, M. Kohno, and C. Bennhold, Phys. Rev. C 39 ,741(1989).

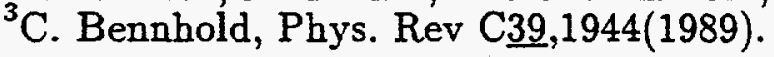


${ }^{4}$ R.A. Williams, C.R. Ji, and S.R. Cotanch, Phys. Rev. C4, 1617(1992).

${ }^{5}$.I. Bockhorst et al., Z. Phys. C, in press, (1994).

${ }^{\circ} \mathrm{H}$. Ito, W.W. Buck and F. Gross, Phys. Lett. 287B,23(1992); W.W. Buck, R. Williams and H. Ito, CEBAF preprint TH-94-2.

H. Ito and F. Gross, Phys. Rev. Lett. 71,2555(1993).

'L.A. Kondratyuk and M.V. Terent'ev, Yad. Fiz. 31,1087(1980); I.G. Aznauryan, A. S. Bagdasaryan and N.L. Ter-Isaakyan, Yad. Fiz. 36,1278(1982); P.L. Chung and F. Coester, Phys. Rev. D44,229(1991).

${ }^{9}$ J.G. Congleton, J. Phys. G18,339(1992). 
PROPOSED

TOPIC: The Weak Decay of Multi-Strange Baryonic Systems

INVESTIGATORS: C. Bennhold, A. Ramos (U. of Barcelona, Spain), D.R. Lehman, and W.C. Parke

OBJECTIVE: To estimate the lifetimes of double- $\Lambda$ bypernuclei and other baryonic systems with $\mathrm{S}<-1$.

SIGNIFICANCE: If all of the information that is available on the NN-, NY-, and YYinteractions is extrapolated to systems with large strangeness $S$, one finds a broad class of objects ${ }^{1}$ composed of nucleons, $\Lambda$ 's, $\Sigma$ 's, and $\Xi$ 's that are stable against particle decay. Filled $\Lambda$ orbitals can block the strong decay channel $\equiv N \rightarrow \Lambda \Lambda$, leading to a small net charge of these systems since the Coulomb repulsion generated by protons can be canceled by $\Xi^{-}$'s. The properties of these systems are comparable to those of hypothetical strange quark matter ("strangelets"). The simplest systems with $\mathrm{S}<-1$ are $\Lambda \Lambda$-hypernuclei, which have been observed in a few cases ${ }^{2}$ and are currently being studied experimentally at Brookhaven. ${ }^{3}$

DESCRIPTION OF PROPOSED WORK: Multi-strange systems that are stable against particle decay will eventually undergo either successive mesonic decays of the hyperons (such as $\Lambda \rightarrow N \pi$ or $\Xi \rightarrow \Lambda \pi$ ) or nonmesonic decays if the mesonic decay channels are Pauli blocked. In order to detect these systems in relativistic heavy ion collisions, one needs to have an estimate of their lifetimes. Based on our experience with the nonmesonic hypernuclear decay, ${ }^{4} \Lambda N \rightarrow N N$, we plan to study decay mechanisms for systems with $S<-1$, such as $\Lambda \Lambda$-hypernuclei. In this case, one finds, besides the usual nonmesonic channels, $\Lambda p \rightarrow n p$ and $\Lambda n \rightarrow n n$, the processes $\Lambda \Lambda$ $\rightarrow$ In, $\Lambda \Lambda \rightarrow \Sigma^{0} \mathrm{n}$, and $\Lambda \Lambda \rightarrow \Sigma \mathrm{p}$. The study of $\Lambda \Lambda$-hypernuclei is relevant with regard to the six quark H-dibaryon, a (ssuudd) composite with $\mathrm{J}=\mathrm{I}=0$ that is postulated to have a mass smaller than two $\Lambda$ 's. Thus, unless the $H$ hides very closely to the $\Lambda \Lambda$ threshold, one should observe a strong decay, such as ${ }_{\Lambda \Lambda}^{6} \mathrm{He} \rightarrow \mathrm{H}+{ }^{4} \mathrm{He}$, rather than a weak decay.

We will begin this project by studying the weak decay of ${ }_{A \Lambda}^{6} \mathrm{He}$. As a first step, we will generalize our present weak one-boson exchange potential for the $\Lambda \mathrm{N} \rightarrow \mathrm{NN}$ reaction, which includes $\pi, \eta, \mathrm{K}, \rho, \omega$, and $\mathrm{K}^{*}$ exchanges, to the process $\Lambda \Lambda \rightarrow \mathrm{YN}$ with $\mathrm{Y}=\Lambda$ or $\Sigma$. The weak vertices will be calculated using $\mathrm{SU}(6)_{\psi}$ symmetry and the pole model, just as in the $\Lambda N \rightarrow N N$ case. Initially, the computations will be performed in a shellmodel framework, and as a second step, we will develop more sophisticated $\alpha \Lambda \Lambda$ threebody wave functions. The partial decay rates for the lambda-induced channel $(\Lambda \Lambda$ $\rightarrow \mathrm{YN}$ ) may be measurable at BNL and could therefore be compared directly to our predictions.

Furthermore, we will explore the nonmesonic decay of other light $\Lambda \Lambda$ hypernuclei, such as ${ }_{A}^{4} \mathrm{H},{ }_{A}^{4} \mathrm{He}$, and ${ }_{{ }^{4} \mathrm{n}} \mathrm{n}$. Four-body hypernuclear structure calculations will have to be performed to verify if these systems are bound and to obtain wave functions. In order to study the decay of systems with $S<-2$, we will generalize the decay process first to $\mathrm{Y}^{\circ} \rightarrow \mathrm{YN}$ (with $\mathrm{Y}=\Lambda$ or $\Sigma$ ) and then to the weak process that can include $\Xi$, such as $N \equiv \rightarrow \mathrm{YN}$ and $\mathrm{Y} \Xi \rightarrow \mathrm{YY}$. A prototype for a system involving $\Xi$ 's that would undergo a weak decay, rather than a strong one (if it is bound), is $\equiv \Omega^{7} \mathrm{He}$ since the strong 
conversion process $\Xi N \rightarrow \Lambda$ is Pauli blocked.

${ }^{1} \mathrm{~J}$. Schaffner et al., Phys. Rev. Lett. $71,1328(1993)$, Ann. Phys., in press (1994).

${ }^{2}$ M. Danysz et al., Nucl. Phys. 49.121(1963); J. Prowse, Phys. Rev. Lett. 17,782(1966).

${ }^{3}$ Brookhaven experiment 855 , (G. Franklin. spokesman) 1993.

${ }^{4}$ A. Ramos, C. Bennhold, E. van Meijgaard and B.K. Jennings. Phys. Lett. 264B,233 (1991); C. Bennhold and A. Ramos, Phys. Rev. C45,3017(1992); A. Ramos, E. van Meijgaard, C. Bennhold and B.K. Jennings, Nucl. Phys. A 544,703(1992); A. Ramos, C. Bennhold, E. van Meijgaard, and B.K. Jennings, Nucl. Phys. A $\underline{54 i} .103 c(1992)$. 
PROPOSED

TOPIC: Cluster-Dynamical Treatment of Pion-Nucleon Scattering and Self-Consistent Determination of the $\pi N \cdot N$ Form Factor

INTESTIGATOR: H. Haberzettl

OBJECTIVE: To obtain a consistent covariant crossing-symmetric numerical solution of the pion-nucleon scattering problem including a self-consistent determination of the off-shell $\pi N-N$ form factor.

SIGNIFICANCE: The results of this problem are not only important in and of themselves, but provide extremely important stepping stones for all few-nucleon systems. Preliminary investigations show, in particular, that the cluster-dynamical approach may be capable of providing an explanation for the somewhat puzzling soft versus hard cutoff requirements of the $\pi N-N$ form factor in the context of the $\pi N$ and $X Y$ problems. If these preliminary results were borne out by a full numerical calculation, this would be of considerable importance for our understanding of mesonnucleon dynamics in general.

This is a continuation of an existing project (see Progress Report, p. 43). 
TOPIC: Gauge-Invariant Covariant Cluster-Dynamical Description of Electromagnetic Processes

INVESTIGATORS: H. Haberzettl and D. R. Lehman

OBJECTIVE: To incorporate electromagnetic processes into the covariant clusterdynamical approach in a gauge-invariant manner.

SIG.IIFICANCE: To date there exists no completely consistent description of the interaction of (real and virtual) photons with strongly interacting systems at relativistic energies. A practical formulation of this problem will have great significance for almost all theoretical work related to existing and future accelerator physics. At present, preliminary investigations into the correct form of the Ward-Takahashi identities in the cluster-dynamical approach exist already. 
TOPIC: Theoretical and Mathematical Questions Related to the Relativistic Cluster Dynamical Approach

INVESTIGATORS: H. Haberzettl, H. Petry, and D. Schütte (both Universität Bonn. Germany)

OBJECTIVE: To clarify mathematical and theoretical questions concerning the relation between the manifestly covariant time-ordered relativistic cluster-dynamical approach and the usual field-theoretical attempts at formulating a relativistic composite-particle theory.

SIGNIFICANCE: A successful clarification of these questions may lead to some deep insights into the origins of the long-standing problems encountered in local field theories. 
PROPOSED

TOPIC: Consistent Cluster-Dynamical Model Description of Quark Propagators and Quark-Gluon Vertices

INVESTIGATORS: C. Bennhold, H. Haberzettl, and H. Ito

OBJECTIVE: To extend the model description of confining quark propagators obtained with the covariant relativistic cluster-dynamical approach to a consistent incorporation of dressed quark-gluon propagators.

SIGNIFICANCE: Although quantum chromodynamics (QCD) is generally held to be the fundamental theory of strong interaction, to date there exists no direct derivation of the dynamical mechanism of quark confinement. A consistent description of confined quarks and dressed quark-gluon vertices in a dynamical model such that it may be utilized for the calculations of mesonic or baryonic scattering processes is highly desirable and may be of significant help for modeling and understanding the dynamical mechanisms of meson-baryon reactions and, in particular, of electromagnetic processes to be measured at, e.g., CEBAF. The findings (reported in the Progress Report, p. 44) that the cluster-dynamical formulation in terms of gluon loops with bare vertices leads to dynamically-confined quarks in a rather straightforward way suggests that the consistent incorporation of quark-gluon loops along the same lines may be capable of providing a model description of the running quark-gluon coupling constant. 
TOPIC: Triton Binding-Energy Calculation with Three-Body Forces and Relativistic Effects (Ph.D. Thesis)

INVESTIGATORS: A. Rakei (Ph.D. Student), H. Haberzettl (Director), and W.C. Parke (Co-Director)

OBJECTIVE: To solve the three-nucleon bound-state problem with the three-body forces found in the cluster-dynamical treatment. ${ }^{1-3}$

SIGNIFICANCE: Triton binding energies calculated with realistic nucleon-nucleon $(N N)$ interactions typically fall short of the experimental value of $8.48 \mathrm{MeV}$ by about 0.5-1 MeV. Attempts to resolve this discrepancy in terms of meson-exchange-based three-body forces provide encouraging but, at present, not entirely satisfactory results. The dynamically most detailed description of meson contributions in the three-nucleon system is provided by the coupled-channel approach pioneered by the Hannover group. ${ }^{4}$ Using the Paris potential, they find that employing explicit $\Delta$ channels raises the binding energy by $0.4 \mathrm{MeV}$ to $7.85 \mathrm{MeV}$, which still is about $0.6 \mathrm{MeV}$ short of the experimental value. The results ${ }^{5}$ found for the one-pion exchange three-body force discussed on pp. 36-37 in this report suggest that the cluster-dynamical treatment may provide a more consistent framework for the calculation of three-body force effects than the usual coupled-channel approaches.

DESCRIPTION OF PROGRESS: The detailed outline of the project is given in Ref. 3 (see also p. 41 in this report). The underlying nucleon-nucleon force to be taken is the Paris potential in a separable PEST expansion ${ }^{6}$. The code for the solution of the nucleons-only problem exists ${ }^{7}$ and will be amended for the inclusion of the three-body forces. The numerical code for describing the $\Delta$ as a dressed resonance according to the prescription of Ref. 1 is essentially completed. At present, the partial-wave analysis of the various contributing diagrams is being undertaken.

${ }^{1}$ H. Haberzettl, Phys. Rev. C499,2142(1994).

${ }^{2} \mathrm{H}$. Haberzettl and W.C. Parke, "Relativistic origin of three-nucleon forces", in Contributed Papers, 14th International IUPAP Conference of Few-Body Problems in Physics (Williamsburg, VA, 26-31 May 1994), edited by Franz Gross (College of William \& Mary/CEBAF, 1994), p. 881; and to be published in Proceedings of the 14th International IUPAP Conference of Few-Body Problems in Physics (Williamsburg, VA, 26-31 May 1994).

${ }^{3} \mathrm{H}$. Haberzettl and W.C. Parke, "Three-nucleon forces, relativity, and cluster dynamics three-nucleon forces", submitted for publication.

${ }^{4}$ Ch. Hajduk, P.U. Sauer, and W. Strueve, Nucl. Phys. A405,581(1983); Ch. Hajduk, P.U. Sauer, and S.N. Yang, ibid. A405,605(1983); M.T. Peña, H. Henning, and P.U. Sauer, Phys. Rev. C42,855(1990).

${ }^{5} \mathrm{H}$. Haberzettl and W.C. Parke, "Cluster-dynamical treatment of three-nucleon forces", in Proceedings of the XIVth European Conference of Few-Body Problems in Physics (Amsterdam, The Netherlands, 23-27 August 1993), edited by B.L.G. Bakker and R. van Dantzig [Few-Body Systems Suppl. 7, 1994], p. 274.

${ }^{6} \mathrm{~J}$. Haidenbauer and W. Plessas, Phys. Rev. C 30,1822 (1984); ibid. 32,1424(1985); J. Haidenbauer, Y. Koike, and W. Plessas, $\underline{\text { ibid. }}$ 33,439(1986); J. Haidenbauer and Y. Koike, ibid. $\underline{34}, 1187(1986)$. 
PROPOSED

'W.C. Parke, Y. Koike, D.R. Lehman, and L.C. Maximon, Few-Body Systems 11.89(1991). 
PROPOSED

TOPIC: Relativistic proton-deuteron scattering and the tensor analyzing power- $T_{20}$

INIESTIGATORS: Hiroshi Ito and Franz Gross (CEBAF and College of William and Mary)

OBJECTIVE: To calculate the cross section and tensor analyzing power of elastic and inelastic $d p$-scattering and to investigate the reaction mechanism within the relativistic Faddeev equation with the spectator nucleon on-massshell.

SIGNIFICANCE: Measurements of the spin-polarization observables in medium-energy nuclear reactions often help us explore new aspects in nuclear dynamics. For example, the Dirac phenomenology ${ }^{1}$ successfully describes the analyzing power of $p A$-scattering, and this indicates the importance of the negative energy states in the field theoretical description $^{2}$ of complex nuclei. In few-body systems, a serious problem remains to be explained. With the beam energy of $0.3-2.4 \mathrm{GeV}$ the measured values of tensor analyzing power, $T_{20}$, in both $d p$-backward elastic ${ }^{3,4,5}$ and inclusive deuteron break$\mathrm{up}^{6.7}$ reactions have a significant deviation from the theoretical predictions based on the nonrelativistic impulse amplitude (IA). The origin of $T_{20}$ is geometrical in the IA, and this simple estimate has a minimum value, $-\sqrt{2}$, and predicts positive values in the higher momentum region. In the experimental data, however, the most negative value is $-0.92 \pm 0.025$ and remains negative. Several authors ${ }^{8-11}$ have investigated the relativistic nucleon-exchange amplitude in backward scattering, ${ }^{1} \mathrm{H}(d, p) d$, including the negative energy state for the exchanged nucleon. Whereas the result is very different from that in the nonrelativistic calculation, the discrepancy still remains. In the case of the deuteron break-up reaction, ${ }^{1} \mathrm{H}(d, p) p n$, the effect of the final-state rescattering processes has been investigated by using the on-shell NN scattering amplitude along with the $d-p-n$ vertex in the nonrelativistic framework. Although inclusion of the rescattering processes improves the result with the IA significantly, the observed values of $T_{20}$ as well as the origin of a shoulder appearing in the differential cross section are not totally explained. But it is clear that the value of $T_{20}$ is sensitive to the rescattering process (a three-body reaction mechanism), where the loop integrals depend on the high-momentum component of the $d$ - $p$-n vertex. It is clearly desirable to attack and solve this problem within a consistent framework using the Faddeev equation. Here, the relativistic approach is mandatory for the kinematical region of significance for this problem. This motivates our proposal.

DESCRIPTION OF PROPOSED WORK: The Bethe-Salpeter equation can be reduced to a three-dimensional form by restricting one of the propagators to be on the massshell ${ }^{12}$, and this procedure still keeps the manifest covariance of the equation. This socalled "spectator on-mass-shell approach" has been successfully applied to the nuclear two-body problem ${ }^{13}$ both for the scattering and bound states, yielding a field theoretical model of the NN interaction and the relativistic deuteron wave function. Within this approach the relativistic three-body scattering equation is no more complex than the non-relativistic Faddeev equation, and the three-body bound state problem has been attacked $^{14}$. We apply this equation to the $d p$ - scattering problems. To find out the important physical mechanisms and not to bury them in the technical complexity of the Faddeev equation, we start with the perturbative evaluation ${ }^{8}$ of amplitudes with the Feynman diagrams by using the relativistic $d-p-n$ vertex and scattering amplitude 
PROPOSED

recently obtained. In the meanwhile, the practical expression of the relativistic threebody scattering equation will be formulated within the helicity base $e^{15}$. This is the general strategy for completing the proposed work within the next three year period:

(i) Calculation of the elastic and inelastic amplitudes from the Feynman diagrams by using the relativistic $d-p-n$ vertex-[1st year].

(ii) Formulation of the three-body scattering equation in the helicity base. A careful procedure of handling the Lorentz boost and the singularities in the equation should be developed-[1st and 2nd year].

(iii) Computer programing of the expression in (ii)-[3rd year].

${ }^{1}$ B.C. Clark, S. Hama, R.L. Mercer, L. Ray and B.D. Serot, Phys. Rev. Lett. 50.1644(1983).

${ }^{2}$ B.D. Serot and J.D. Walecka, Advanced in Nuclear Physics, Vol. 16, eds. J.W. Negele and E. Vogt (Plenum, New York, 1986); and the references therein.

${ }^{3} \mathrm{~J}$. Arvieux, et al., Phys. Rev. Lett. $\underline{50}, 19(1983)$.

${ }^{4} \mathrm{~V}$. Punjabi, et al., Proceedings of the 14th International IUPAP Conference on FewBody Problems in Physics (Williamsburg, VA, May 1994).

${ }^{5}$ L.S. Azhgirey, et. al., ibid.

${ }^{6}$ C.F. Perdrisat, et. al., Phys. Rev. Lett. $59,2840(1987)$.

'V. Punjabi, et al., Phys. Rev. C39,608(1989).

${ }^{8}$ B.D. Keister and J.A. Tjon, Phys. Rev. C26,578(1982); B.D. Keister, Phys. Rev. C24, 2628(1981).

${ }^{9}$ E.A. Remler, Nucl. Phys. B42,56; 69(1972).

${ }^{10} \mathrm{~F}$. Gross, in Few-Body Dynamics, edited by A. N. Mitra et al. (North-Holland, Amsterdam, 1976), p.523.

${ }^{11} \mathrm{~S}$. Morioka and T. Ueda, ibid. p.297.

${ }^{12}$ F. Gross, Phys. Rev. 186, 1448(1969); C26,2203(1982).

${ }^{13} \mathrm{~F}$. Gross, J. W. Van Orden, and K. Holinde, Phys. Rev. C455,2094(1992).

${ }^{14} \mathrm{~A}$. Stadler and F. Gross, Proceedings of the 14th International IUPAP Conference on Few-Body Problems in Physics (Williamsburg, VA, May 1994).

${ }^{15}$ M. Jacob and G.C. Wick, Ann. Phys. (N.Y.) I,404(1959); J. J. Kubis, Phys. Rev. $\mathrm{D} \underline{6}, 547(1972)$. 
PROPOSED

TOPIC: Wide-angle Bremsstrahlung

INVESTIGATOR: L.C. Maximon

OBJECTIVE: To derive an expression for wide-angle bremsstrahlung (integrated over final electron angles) that is applicable to the analysis of high energy electron scattering experiments.

SIGNIFICANCE: The design of detectors for high energy electron accelerators requires the calculation of background due to wide-angle bremsstrahlung at high energies. The calculations generally available for high energy electrons are only valid for photon production at small angles. We will derive both an exact expression, expressed in a form that facilitates numerical calculation (exact within the assumption of one-photon exchanged between electron and nucleus) and a relatively simple analytic expression, valid for the conditions of particular interest in the design of a large-angle spectrometer, viz., high energy for the incident electron, low energy for the emitted photon, and large angles of emission of the photon relative to the incident beam direction. This analytic expression permits one to see very clearly the behavior of the cross section with respect to the parameters of interest: incident electron energy, photon energy, and photon emission angle. Because of its analytic simplicity, this high energy approximation permits one to estimate the cross section for wide-angle bremsstrahlung by scaling the cross sections measured at lower energies.

DESCRIPTION OF PROPOSED WORK: The starting point for this calculation is the completely differential bremsstrahlung cross section including kinematic recoil, in the one photon exchange approximation. Dynamic recoil effects such as photon emission by the target nucleus, and dispersion corrections, involving two photon exchanges and excitation of the nucleus in the intermediate state, will be neglected. This cross section will be integrated over final electron directions using the method of piece-wise analytical integration, applied previously to the calculation of the radiative tail to high energy electron scattering, where one integrates over the directions of the emitted photon. ${ }^{1}$ As observed in that work, this method avoids completely the problems related to the presence of sharp peaks in the bremsstrahlung cross section, reduces the time of calculation by several orders of magnitude, and greatly increases the accuracy of the entire calculation. We will also derive a high energy approximation to the cross section for wide angle bremsstrahlung. We will consider in particular the case in which the energy of the emitted photon is small relative to the incident electron energy. With these approximations we can identify clearly the regions in the integration over scattered electron directions which make significant contributions to the cross section. The simplicity of the resulting expression permits one to make estimates of high energy cross sections by scaling measurements made at much lower energies.

${ }^{1}$ L.C. Maximon and S.E. Williamson, Nucl. Instr. and Meth. in Physics Res. A258,95(1987) 
PROPOSED

TOPIC: Radiative Correction to Elastic Electron-Proton Scattering Including Proton Form Factors

INVESTIGATORS: L.C. Maximon and John Tjon

OBJECTIVE: To derive analytic expressions and computer programs for the radiative correction to elastic electron-nucleon scattering that include nucleon form factors for all photon-nucleon interactions, without making the usual soft-photon approximations.

SIGNIFICANCE: The accuracy of the nuclear information obtained from high energy electron scattering experiments is dependent to a large degree on the accuracy of the radiative corrections which must be applied to the measured cross sections. The theoretical expressions generally used for the radiative corrections are all based on the work of Tsai, ${ }^{1}$ who calculated the radiative cross section to high energy electron-proton scattering at large momentum transfers (large compared to the electron rest mass). His calculation included all of the required diagrams (vertex corrections for the electron and for the proton, self-energy diagrams, vacuum polarization, box and crossed box diagrams, as well as the emission of soft real photons by both the electron and the proton). However, throughout that work the approximation was made that all photons (real and virtual) connected to the proton were soft (with the exception of the one hard photon involved with the scattering). With this approximation, no effects of the proton structure are included in the radiative correction; for photons connected to the proton lines only the infrared divergent terms are retained, and these cancel in the final result, as they should. Terms of the order of $1 \%$ are neglected throughout. While the question of the accuracy of the currently used radiative correction has been discussed in several published papers, the proposed work will constitute the first complete calculation that includes nucleon form factors and makes no soft photon approximations for the virtual photons. It will thus provide both a measure of the accuracy of previous calculations and a reliable starting point for the more difficult calculation of the radiative corrections for coincidence experiments.

DESCRIPTION OF PROPOSED WORK: The Feynman diagrams comprising the radiative correction are well-known. The proton will be taken to be a Dirac particle with an anomalous magnetic moment and on-shell form factors of a dipole form. With these assumptions, the integrals over the virtual photon momenta may all be performed analytically. The resulting expressions will then be examined in the limit of high energies and large momentum transfers, at which point comparison may be made with the radiative correction that is used generally. (It should be noted that while the momentum transfer is always much larger than the electron rest mass for the high energy experiments of interest, it may, however, be of the same order of magnitude as the nucleon rest mass.) Computer programs will be written to complement and provide a check on the analytic calculations. They also provide the possibility of using nucleon form factors of a more general form. Certain model dependences of the radiative correction on the nucleon structure will be studied.

${ }^{1}$ Y.S. Tsai, Phys. Rev. 122,1898(1961);

L. W. Mo and Y.S. Tsai, Rev. Mod. Phys. 41,205(1969);

Y.S. Tsai, SLAC - PUB - 848, January 1971 
TOPIC: $\quad \alpha+d \rightarrow{ }^{6} \mathrm{Li}+\gamma$ Scattering Cross Section at Threshold Energies

INIESTIGATORS: W.C. Parke and Ana Eiro (U. of Lisbon)

OBJECTIVE: To calculate the low-energy cross section for the reaction $\alpha+\mathrm{d} \rightarrow$ ${ }^{6} \mathrm{Li}+\gamma$ using consistent three-body dynamics in both the initial and final states and our new technique for handling the Coulomb interaction

SIG.NIFICANCE: As described in the Progress Report, p. 75, understanding the radiative capture of deuterons on alpha particles has fundamental importance in astrophysics and nuclear physics. Cross sections near threshold (at energies corresponding to fusion and stellar interior temperatures as well as cosmic-ray spallation energies) are extremely difficult to measure, but are needed in reaction rate calculations. Extrapolation from higher energies is notoriously dangerous, particularly when threshold resonances, enhanced capture into a small component of the ground state, and three-body effects are missed. Thus, a solid theoretical description is needed.

DESCRIPTION OF PROPOSAL: We plan to extend the full three-body calculation for the radiative capture process to include the long-range Coulomb repulsion between the $\alpha$ particle and the proton, an effect needed for center-of-mass energies below a few hundred $\mathrm{keV}$. The first stage is to extend the ${ }^{6} \mathrm{Li}$ bound-state three-body code to include a long-range force. We will use the method of Koike (see Progress Report, p. 78 , for a description of this method) to avoid the convergence problems usually associated with calculating the Coulomb-modified observables. For the rescattering corrections to the capture amplitude, the finite range of the bound-state will naturally truncate many of the otherwise long-range terms. For the intermediate rescattering terms in the three-body equations corresponding to the exchange of photons rather than a baryonic cluster, the full Coulomb t-matrix can be employed. 